\title{
Numerical investigation of the influence of elevated turbulence levels on the cooling effectiveness of an anti-vortex hole geometry
}

Timothy William Repko

West Virginia University

Follow this and additional works at: https://researchrepository.wvu.edu/etd

\section{Recommended Citation}

Repko, Timothy William, "Numerical investigation of the influence of elevated turbulence levels on the cooling effectiveness of an anti-vortex hole geometry" (2014). Graduate Theses, Dissertations, and Problem Reports. 391.

https://researchrepository.wvu.edu/etd/391

This Thesis is protected by copyright and/or related rights. It has been brought to you by the The Research Repository @ WVU with permission from the rights-holder(s). You are free to use this Thesis in any way that is permitted by the copyright and related rights legislation that applies to your use. For other uses you must obtain permission from the rights-holder(s) directly, unless additional rights are indicated by a Creative Commons license in the record and/ or on the work itself. This Thesis has been accepted for inclusion in WVU Graduate Theses, Dissertations, and Problem Reports collection by an authorized administrator of The Research Repository @ WVU. For more information, please contact researchrepository@mail.wvu.edu. 


\title{
NUMERICAL INVESTIGATION OF THE INFLUENCE OF Elevated Turbulence LeVels ON THE COOling EFFECTIVENESS OF AN ANTI-VORTEX HOLE GEOMETRY \\ by \\ Timothy William Repko
}
Thesis submitted to College of Engineering and Mineral Resources at West Virginia University in partial fulfillment of the requirements for the degree of

Master of Science

in

Aerospace Engineering

\author{
Approved By: \\ Andrew C. Nix, Ph.D., Chair \\ John Kuhlman, Ph.D. \\ Wade Huebsch, , Ph.D. \\ Department of Mechanical and Aerospace Engineering \\ Morgantown, West Virginia \\ May 2014
}

Keywords: Film Cooling, Freestream Turbulence, CFD, Heat Transfer Copyright (C) 2014, Timothy W. Repko 


\begin{abstract}

\section{NuMERICAL INVESTIGATION OF THE INFLUENCE OF Elevated Turbulence Levels on the CoOling EFFECTIVENESS OF AN ANTI-VORTEX HOLE GEOMETRY}

Timothy William Repko

A novel film cooling hole geometry for use in gas turbine engines has been investigated numerically by solving the Reynolds Averaged Navier-Stokes equations in a commercial CFD code (STAR-CCM+) with varying turbulence intensity and length scale using the $k$ $\omega$ SST turbulence model. Both steady and unsteady results were considered in order to investigate the effects of freestream turbulence intensity and length scale on this novel anti-vortex hole (AVH) concept. The AVH geometry utilizes two side holes, one on each side of the main hole, to attempt to mitigate the vorticity from the jet from the main hole. The AVH concept has been shown by past research to provide a substantial improvement over conventional film cooling hole designs. Past research has been limited to low turbulence intensity and small length scales that are not representative of the turbulent flow exiting the combustor. Three turbulence intensities $(\mathrm{Tu}=5,10$ and 20\%) and three length scales normalized by the main cooling hole diameter $\left(\Lambda_{x} / d_{m}=1,3,6\right)$ were considered in this study for a total of nine turbulence conditions. The highest intensity, largest length scale turbulence case $\left(\mathrm{Tu}=20, \Lambda_{\mathrm{x}} / \mathrm{d}_{\mathrm{m}}=6\right)$ is considered most representative of engine conditions and was shown to have the best cooling performance. Results show that the turbulence in the hot gases exiting the combustor can aid in the film cooling for the AVH geometry at high blowing ratios $(\mathrm{BR}=2.0)$, where the blowing ratio is essentially the ratio of the jet-to-mainstream mass flux ratios. Length scale was shown to have an insignificant effect on the cooling performance at low turbulence intensity and a moderate effect at higher turbulence intensities. The adiabatic film cooling effectiveness was shown to increase as the turbulence intensity was elevated. The convective heat transfer coefficient was also shown to increase at the turbulence intensity was elevated. An increase in the heat transfer coefficient is a deleterious effect and must be weighed against the improvements in the adiabatic cooling effectiveness. The net heat flux reduction (NHFR) is the parameter used to quantify the net benefit of film cooling. As a general trend, the NHFR was shown to increase with the turbulence intensity in all cases. 


\section{ACKNOWLEDGEMENTS}

"Don't be too timid and squeamish about your actions. All life is an experiment. The more experiments you make the better."

Ralph Waldo Emerson

First and foremost I would like to thank my parents. Without them college would have been much tougher, if not impossible.

Seven years I have been at WVU, including my undergraduate career. Throughout this time many people have helped me get to where I am today. My advisor, Dr. Nix, hired me as an undergraduate research assistant and gave me the guidance necessary to direct me towards graduate school here at WVU. With Dr. Nix's help, guidance, and support I was able to complete my Masters work in a reasonable time although I took two summers off to pursue a UTSR fellowship at Solar Turbines and an internship at Johns Hopkins Applied Physics Lab. The support and friendship of Dr. Nix has been instrumental in my development as an engineer.

Much of my knowledge in fluid dynamics and turbulence can be attributed to the classes taught by Dr. Kuhlman. None of the classes I took that were taught by Dr. Kuhlman could be considered easy, but they were always well taught and interesting. It is a rare skill to be able to keep a class attentive and intrigued during lengthy derivations and problems. I still use my notes for many of theses classes as a reference.

I would also like to thank my third committee member, Dr. Huebsch. He was able to help me with my CFD work or direct me to someone who could.

Thanks to my long time friend and fellow graduate student, Steve Hayes. His help in my work has been invaluable. He has been able to help me look at problems in different ways time and time again.

Thanks to everyone else that worked in the office; Kevin Luo, Josh Matheny, Cesar Sandoval, John Hailer, Evan Ford, Dustin Frohnapfel, Chad Jones, Josh Everett, and Joe Close (even though he didn't work in the office, he was there enough). I'd also like to thank Dan Carder and Jay Wilhelm. It was nice to have people there to break up the monotony of CFD work. 


\section{CONTENTS}

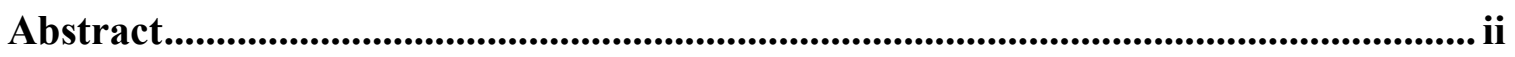

Acknowledgements ........................................................................................................... iii

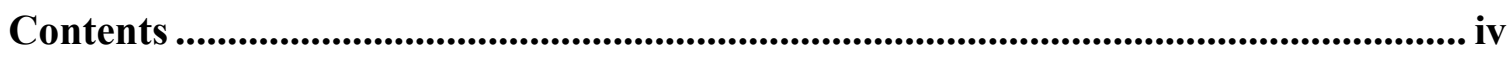

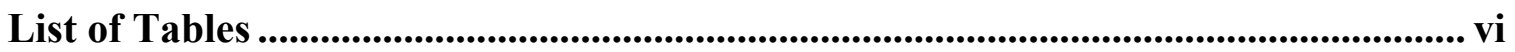

List of Figures............................................................................................................................... vii

Nomenclature ....................................................................................................................... xi

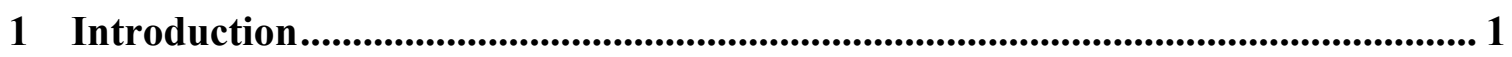

1.1 Gas Turbine Engines....................................................................................... 1

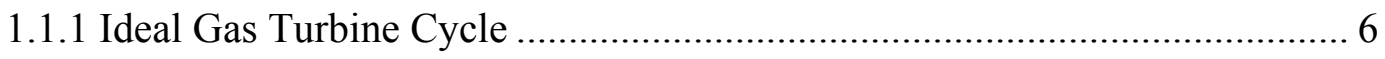

1.1.2 Real Gas Turbine Cycle ............................................................................ 10

1.2 Gas Turbine Cooling and Heat Transfer ............................................................. 12

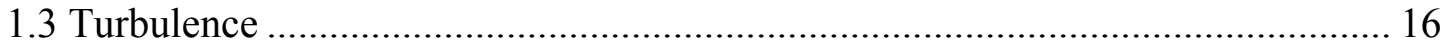

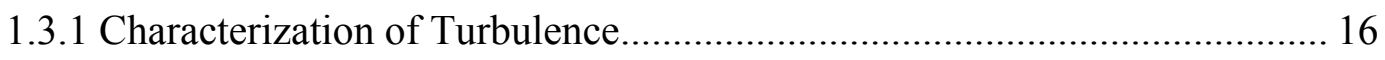

1.3.2 Signal decomposition, Turbulence Intensity and Length Scale................... 20

1.4 Overview of Current Work and Problem Statement............................................. 22

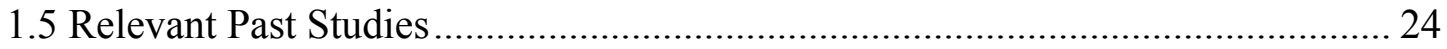

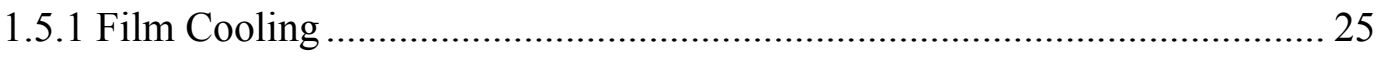

1.5.2 Engine Conditions and Effects of Turbulence …………............................. 31

2 Computational and Experimental Setups..................................................................... 33

2.1 RANS and Description of Closure Problem .......................................................... 34

2.1.1 Selection of Turbulence Model..................................................................... 35

2.2 Control Volume and Computational Setup............................................................. 38

2.2.1 Original RANS Cases and Heat Transfer Cases .......................................... 40

2.2.2 Flat Plate Study .......................................................................................... 43

2.2.3 Unsteady RANS Cases ......................................................................... 44

2.2.4 Additional Cases to Match Future Experimental Work at WVU ................. 46 


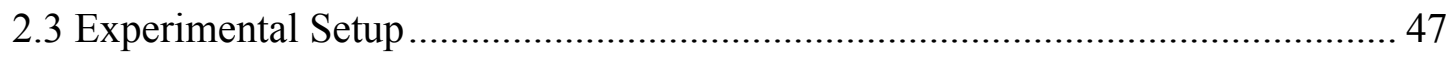

3 Analysis and Results ................................................................................................... 51

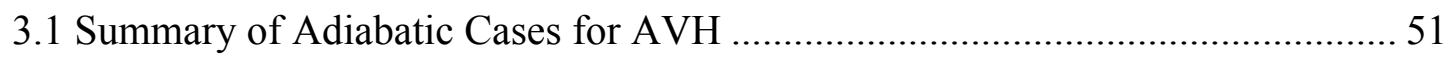

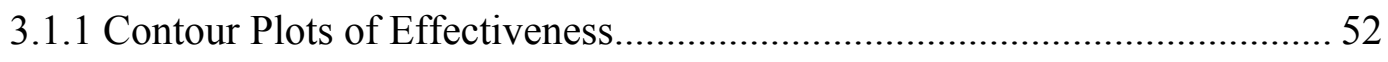

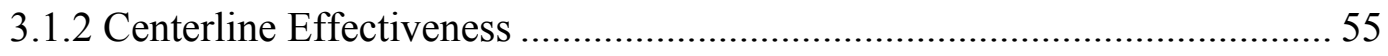

3.1.3 Span-Averaged Effectiveness ................................................................... 57

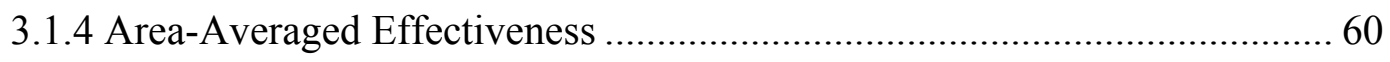

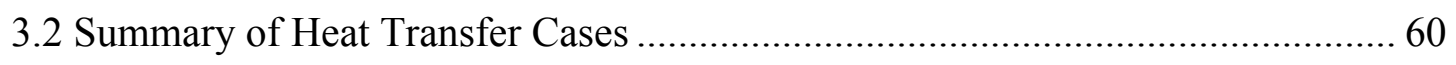

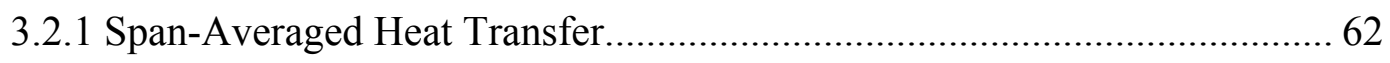

3.2.2 Area-Averaged Heat Transfer...................................................................... 66

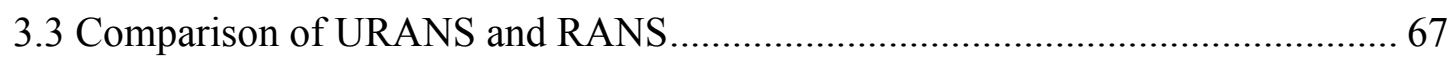

3.3.1 Secondary Velocity Vectors Downstream of Cooling Holes ........................ 68

3.3.2 Unsteadiness in the Wake of the Main Cooling Jet ....................................... 71

3.3.3 Comparison of URANS and RANS Temperature Field Prediction.............. 76

3.3.4 Mixing of Jets, Jet Lift off, and Lateral Spreading ....................................... 79

4 Summary and Conclusions ……........................................................................................... 81

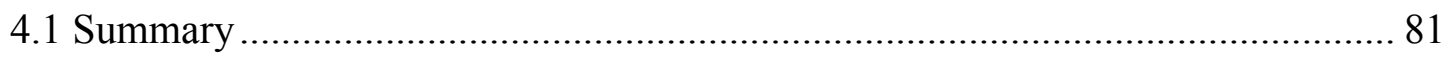

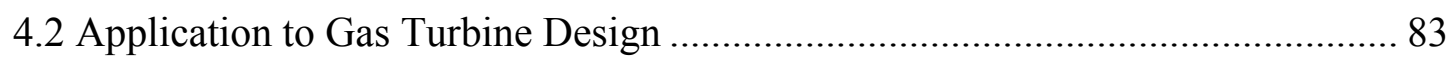

4.3 Future and Concurrent Work ........................................................................... 85

Appendices................................................................................................................................ 87

Appendix A: Summer Heat Transfer Conference Paper (2013)................................. 87

Appendix B: Draft of ASME Journal Of Turbomachinery Publication ...................... 98

Appendix C: Matlab Code for Experimental Work ................................................... 110

References.............................................................................................................................. 114 


\section{LIST OF TABLES}

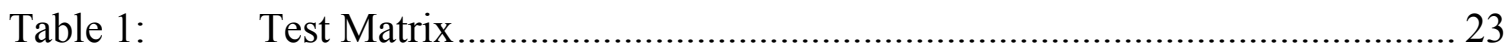

Table 2: $\quad$ Geometric Parameters For Tested Version AVH ................................... 30

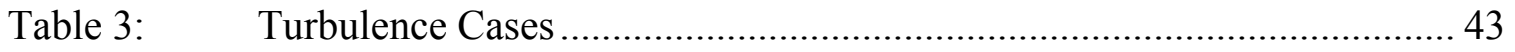

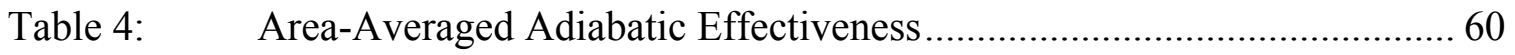

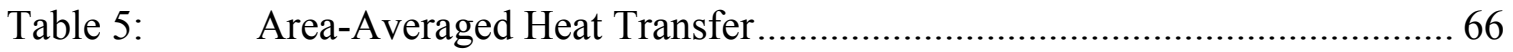




\section{LIST OF FigURES}

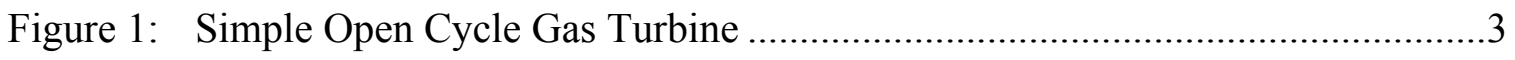

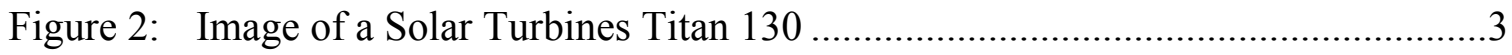

Figure 3: Cutaway View of a GE TF34 9000-Pound Class Turbofan Engine

(Courtesy GE [4] ) ............................................................................

Figure 4: T-s Diagram for Ideal Gas Turbine Cycle .......................................................6

Figure 5: Thermal Cycle Efficiency of a Simple Gas Turbine Varying With

Pressure Ratio and Turbine Inlet Temperature ................................................

Figure 6: Comparison T-s Diagram of Real and Ideal Brayton Cycles ..........................11

Figure 7: Specific Core Power Production Compared to Turbine Inlet

Temperature for Historic Engines and Projections to Future [5]......................12

Figure 8: Diagram the Development of a Cooled Turbine Blade [6] ..............................14

Figure 9: Turbulent Velocity Fluctuation..............................................................18

Figure 10: Schematic Representation of an Intermittent Flow[8] ..................................20

Figure 11: Reynolds Decomposition of Turbulent Velocity Signal [10] .........................21

Figure 12: Diagram Depicting The Counter Rotating Vortex (CRV) Problem

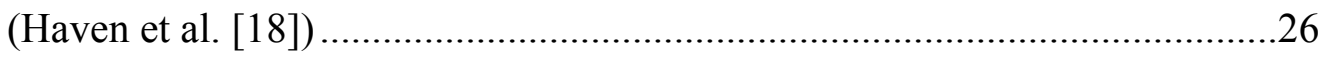

Figure 13: Coupons Showing Geometries of Various Film Cooling Geometries

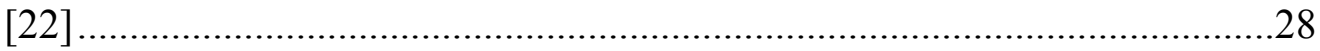


Figure 14: Three View Drawing of Anti-Vortex Hole (AVH)......................................30

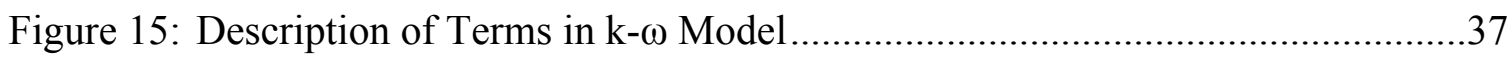

Figure 16: Isometric and Side View of CFD Control Volume Compared to Wind

Tunnel .38

Figure 17: Front, Side, and Top View of Control Volume Modeled For Steady

RANS . .40

Figure 18: Computational Grid for Adiabatic and Heat Transfer Cases.

Figure 19: Flat Plate Nusselt Number Comparison.....................................................44

Figure 20: URANS Mesh With Volumetric Controls ..............................................45

Figure 21: CAD Drawing of Experimental Facility ...................................................47

Figure 22: Transient Heat Transfer With Convection and Semi-Infinite Solid Assumption .48

Figure 23: Typical Film Cooling Coverage of Straight Film Cooling Hole at High Blowing Ratio $(\mathrm{BR}=2)[43]$ 53

Figure 24: Contour Plots of Adiabatic Effectiveness for Nine Turbulence

Conditions .54

Figure 25: Centerline Adiabatic Effectiveness at Constant Length Scale (a-c) and Constant Turbulence Intensity (d-f). .56

Figure 26: Span-Averaged Adiabatic Effectiveness at Constant Length Scale (a-c) and Constant Turbulence Intensity (d-f) 
Figure 27: Span-Averaged Dimensionless Heat Transfer Coefficients at Constant Length Scale (a-c) and Constant Turbulence Intensity (d-f)

Figure 28: Span-Averaged NHFR at Constant Length Scale (a-c) and Constant Turbulence Intensity (d-f) .65

Figure 29: Velocity Vectors Tangential to the Freestream at $x / d_{m}=3$ and $x / d_{m}=7$

(RANS - Right, URANS - Left, Tu $=20 \%$, and $\Lambda_{\mathrm{x}} / \mathrm{d}_{\mathrm{m}}=1$ )

Figure 30: Velocity Vectors Tangential to the Freestream at $\mathrm{x} / \mathrm{d}_{\mathrm{m}}=15$ and $\mathrm{x} / \mathrm{d}_{\mathrm{m}}=25$ (RANS - Right, URANS - Left, $\mathrm{Tu}=20 \%$, and $\Lambda_{\mathrm{x}} / \mathrm{d}_{\mathrm{m}}=1$ )

Figure 31: Velocity Vector at $\mathrm{x} / \mathrm{d}=3$ showing Region of Increased Heat Transfer Between Holes (URANS Only)

Figure 32 Comparison of RANS and URANS Predictions of Adiabatic Film Cooling Effectiveness

Figure 33: Absolute Difference Between Quasi-Instantaneous URANS and Time Averaged URANS Film Effectiveness $\left(\mathrm{Tu}=10 \%, \Lambda_{\mathrm{x}} / \mathrm{d}_{\mathrm{m}}=1\right)$ .73

Figure 34: Velocity Vectors Inside of a Film Cooling Hole With Overlaid Schematic Showing Area of Recirculation Leylek et al. [48] .74

Figure 35: Line Integral Convolution of Main Hole Centerline at Four Time Levels Showing Pulsating Flow $\left(\mathrm{Tu}=20 \%, \Lambda_{\mathrm{x}} / \mathrm{d}_{\mathrm{m}}=1\right)$ .75

Figure 36: Isosurface of Temperature Showing Mixing of Coolant Jets For a Turbulence Intensity of $10 \%$ at 50 Time Step Intervals .76 
Figure 37: Dimensionless Temperature Along Main Hole Centerline ( $\mathrm{Tu}=10 \%$,

$$
\left.\Lambda_{\mathrm{x}} / \mathrm{d}_{\mathrm{m}}=1\right)
$$

Figure 38: Comparison of Span-Averaged Film Effectiveness for RANS and

URANS $\left(\mathrm{Tu}=10 \%, \Lambda_{\mathrm{x}} / \mathrm{d}_{\mathrm{m}}=1\right)$

Figure 39: Isosurface of Dimensionless Temperature For URANS Simulations at

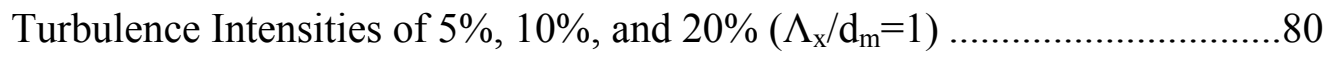




\section{NomenClature}

\begin{tabular}{|c|c|}
\hline BR & blowing ratio or mass flux ratio $(\rho \mathrm{V})_{c} /(\rho \mathrm{V})_{\text {in }}$ \\
\hline c & blade chord length \\
\hline$c_{\mathrm{p}}$ & specific heat at constant pressure \\
\hline$d$ & film cooling hole diameter \\
\hline DR & density ratio $\rho_{c} / \rho_{\text {in }}$ \\
\hline $\mathrm{F}$ & force \\
\hline $\mathrm{g}$ & gravitational constant \\
\hline $\mathrm{h}$ & enthalpy (in Chapter 1) \\
\hline $\mathrm{h}$ & heat transfer coefficient (in Chapter 2) \\
\hline $\mathrm{h}_{\mathrm{f}}$ & film cooling heat transfer coefficient \\
\hline $\mathrm{k}$ & turbulent kinetic energy \\
\hline $\mathrm{F}$ & force \\
\hline $\mathrm{L}$ & arbitrary length \\
\hline$\dot{m}$ & mass flow rate \\
\hline $\mathrm{N}$ & number of elements \\
\hline $\mathrm{Nu}$ & Nusselt number \\
\hline $\mathrm{P}$ & pressure \\
\hline $\operatorname{Pr}$ & Prandtl number \\
\hline $\mathrm{P}_{\mathrm{k}}$ & a production term of $\mathrm{k}$ in $\mathrm{k}-\omega$ turbulence model \\
\hline $\mathrm{P}_{\omega}$ & a production term of $\omega$ in $\mathrm{k}-\omega$ turbulence model \\
\hline$P_{\omega, 1}$ & a production term of $\omega$ in $k-\omega$ turbulence model (SST) \\
\hline $\mathrm{P}_{\omega, 2}$ & a production term of $\omega$ in $k-\omega$ turbulence model (SST) \\
\hline $\operatorname{Re}$ & Reynolds number \\
\hline $\mathrm{S}$ & wetted area \\
\hline $\mathrm{St}$ & Stanton number \\
\hline$S_{i j}$ & mean strain rate tensor \\
\hline
\end{tabular}




$\begin{array}{ll}\mathrm{S}_{\Phi} & \begin{array}{l}\text { Source term for scalar quantity } \\ \text { temperature }\end{array} \\ \mathrm{T} & \text { time } \\ \mathrm{t} & \text { turbulence intensity } \\ \mathrm{Tu} & \text { velocity } \\ \mathrm{U}, \mathrm{V} & \text { fluctuating velocity component } \\ \mathrm{u} & \text { heat transfer rate } \\ \dot{Q} & \text { heat transfer rate per unit mass flow } \\ \mathrm{q} & \text { dynamic pressure } \\ \mathrm{q}_{\infty} & \text { work } \\ \dot{W} & \text { work per unit mass flow } \\ \mathrm{w} & \text { coordinate directions as defined in text } \\ \mathrm{x}, \mathrm{y}, \mathrm{z} & \text { dimensionless wall unit } \\ \mathrm{y}^{+} & \end{array}$

\section{Greek}

$\alpha$

$\beta^{*}$

$\beta_{1}$

$\Gamma$

$\gamma_{1}$

$\gamma_{2}$

$\delta_{\mathrm{ij}}$

$\eta$

$\eta_{\text {aw }}$

$\eta_{\mathrm{k}}$

$\Theta$

$\theta$ thermal diffusivity

a constant in the $\mathrm{k}-\omega$ turbulence model

a constant in the $\mathrm{k}-\omega$ turbulence model

a constant in the $\mathrm{k}-\omega$ turbulence model

a constant in the $\mathrm{k}-\omega$ turbulence model (SST)

Kronecker delta $\delta_{i j}= \begin{cases}0 & \text { if } i \neq j \\ 1 & \text { if } i=j\end{cases}$

efficiency

adiabatic effectiveness

Kolmogorov length scale

dimensionless temperature $\left(\Theta=\frac{T_{\infty}-T}{T_{\infty}-T_{C}}\right)$

dimensionless temperature $\left(\theta=\frac{T_{\infty}-T_{C}}{T_{\infty}-T_{w}}\right)$ 


$\begin{array}{ll}\mu & \text { dynamic viscosity } \\ v & \text { kinematic viscosity } \\ \Lambda_{\mathrm{x}} & \text { streamwise integral length scale } \\ \rho & \text { density } \\ \sigma_{\mathrm{k}} & \text { a constant in the k- } \omega \text { turbulence model } \\ \sigma_{\omega, 1} & \text { a constant in the } \mathrm{k}-\omega \text { turbulence model (SST) } \\ \sigma_{\omega, 2} & \text { a constant in the k- } \omega \text { turbulence model (SST) } \\ \tau_{\mathrm{w}} & \text { shear stress at the wall } \\ \Phi & \text { mean scalar quantity } \\ \varphi & \text { fluctuating scalar quantity } \\ \omega & \text { specific dissipation } \varepsilon / \kappa\end{array}$

\section{Subscripts/Superscripts}

a

aw

adiabatic wall conditions

bleed

bleed air flow from compressor

c

coolant conditions

comp

compressor condition

$\mathrm{i}, \mathrm{j}, \mathrm{k}$

tensor notation

in

freestream inlet conditions

film

local temperatures in freestream affected by film cooling

$\mathrm{m}$

main hole

RMS

root mean square

RSM

Reynolds Stress Model

S

side hole or AVH

turbulent

turb

turbine condition

W

wall condition

0

no film cooling condition 
$1,2,3,4$

$\bar{x}$

\section{Abbreviations}

AVH

CAD

CFD

CFL

CRV

CMAS

CPU

FLOPS

IGCC

IR

LES

NHFR

NOx

PIV

RAM

RANS

SST stations in Brayton cycle

arithmetic mean

anti-vortex hole

computer aided design

computational fluid dynamics

Courant-Friedrichs-Lewy condition

counter rotating vortex

calcium-magnesium-alumino-silicate

central processing unit

floating operations per second

integrated gasification combined cycle

infrared

large eddy simulations

net heat flux reduction

mono-nitrogen oxides ( $\mathrm{NO}, \mathrm{NO}_{2}$, etc.)

particle image velocimetry

random access memory

Reynolds averaged Navier-Stokes

Shear Stress Transport version of k- $\omega$ turbulence model 


\section{INTRODUCTION}

\subsection{GAS TuRbine ENGINES}

Much like any other prime mover, a gas turbine in its simplest form converts fuel or heat into useful work. The origins of gas turbine engines can be traced back to the $16^{\text {th }}$ century when Leonardo da Vinci sketched a device, the chimney jack, which used hot gases flowing up a chimney to rotate a shaft. The next major development in the history of gas turbine engines was when an English inventor, John Barber, obtained a patent for the first gas turbine. Barber's turbine was to burn wood, coal, or oil to provide the fuel and utilize a reciprocating compressor; limited by the technology of his day Barber was not able to build a prototype to produce a net work output. Others attempted to build a gas turbine engine producing a net work output but none were successful until the $20^{\text {th }}$ century.

Practical applications of the gas turbine engine were refined circa early $20^{\text {th }}$ century when Sir Frank Whittle of England and Dr. Hans von Ohian of Germany were both developing their own prospective versions of a gas turbine engine. Both are recognized as co-inventors of the jet engine, and neither were aware of the others work but each was able to fly his own jet powered aircraft before the end of World War II. An aircraft 
company, Ernst Heinkel Aircraft, is credited with the first flight of an aircraft powered by a turbojet engine. The aircraft was the HE178 flown above Germany in August of 1939 [1].

Developed nearly concurrently with the turbojet used to power the aircrafts mentioned above, a land-based gas turbine was first used in Neuchâtel, Switzerland in 1939 to produce electricity. A. B. Brown Boveri developed this engine in 1939 in Baden, Switzerland and installed it in Neuchâtel, Switzerland. This engine operated on a simple cycle and was the first commercial power-generation turbine in operation and ran until it was retired in 2002 [2].

Regardless of whether a gas turbine is used for aircraft propulsion or electricity generation, the basic principles of gas turbine theory apply. In order for expansion through the turbine to occur, there must first be compression of the working fluid. Simply compressing the working fluid would provide no excess energy when expanding through the turbine so there must be additional energy added to the working fluid. When the working fluid is air, heat is generally added through combustion of a fuel, raising the temperature of the working fluid entering the turbine. By expanding the hot combustion gasses through the turbine, energy can be extracted to run the compressor and the excess energy can be used either to run a generator or produce a propulsive thrust for an aircraft. Three components, the compressor, the combustor, and the turbine, are connected together to make up a simple open cycle gas turbine as shown schematically in Figure 1. These three components make up what is commonly referred to as the gas generator. 


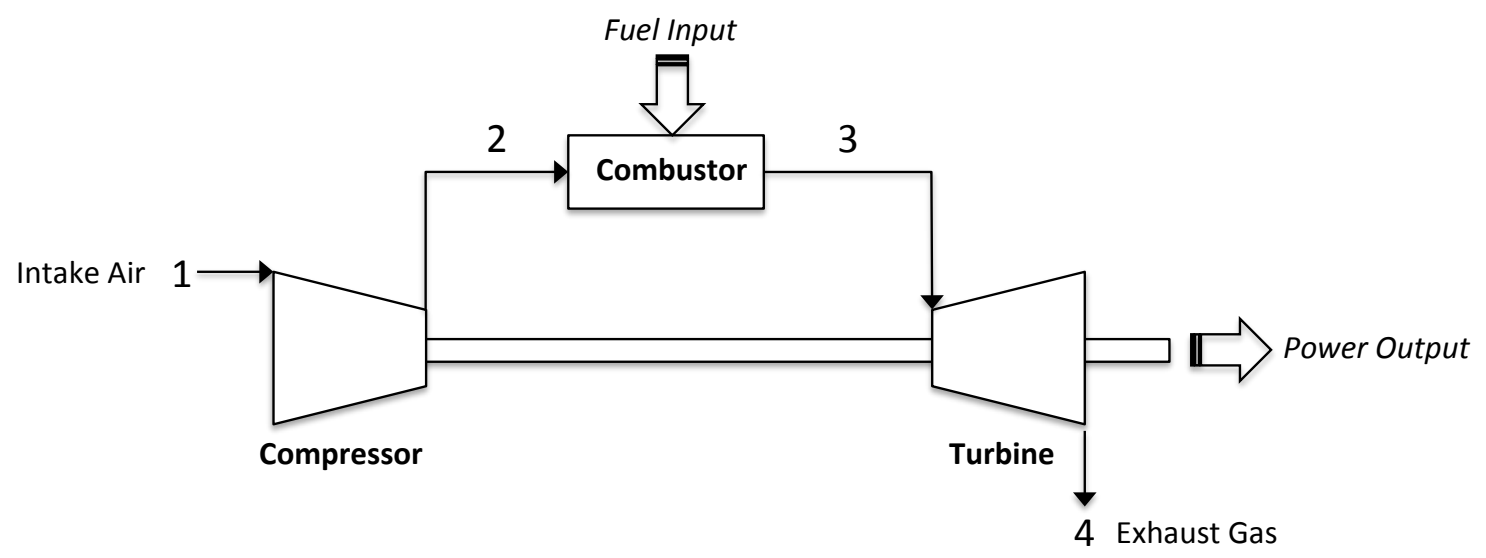

Figure 1: Simple Open Cycle Gas Turbine

The gas generator is essentially the same between a power generation turbine and an aircraft engine. For power generation applications, all of the energy is in stored in the form of heat in the turbine exhaust and is attempted to be recovered by expansion through a second turbine or other means in more complex cycles in order to improve the overall efficiency of the system. Figure 2 is an example of a land based power generation turbine produced by Solar turbines.

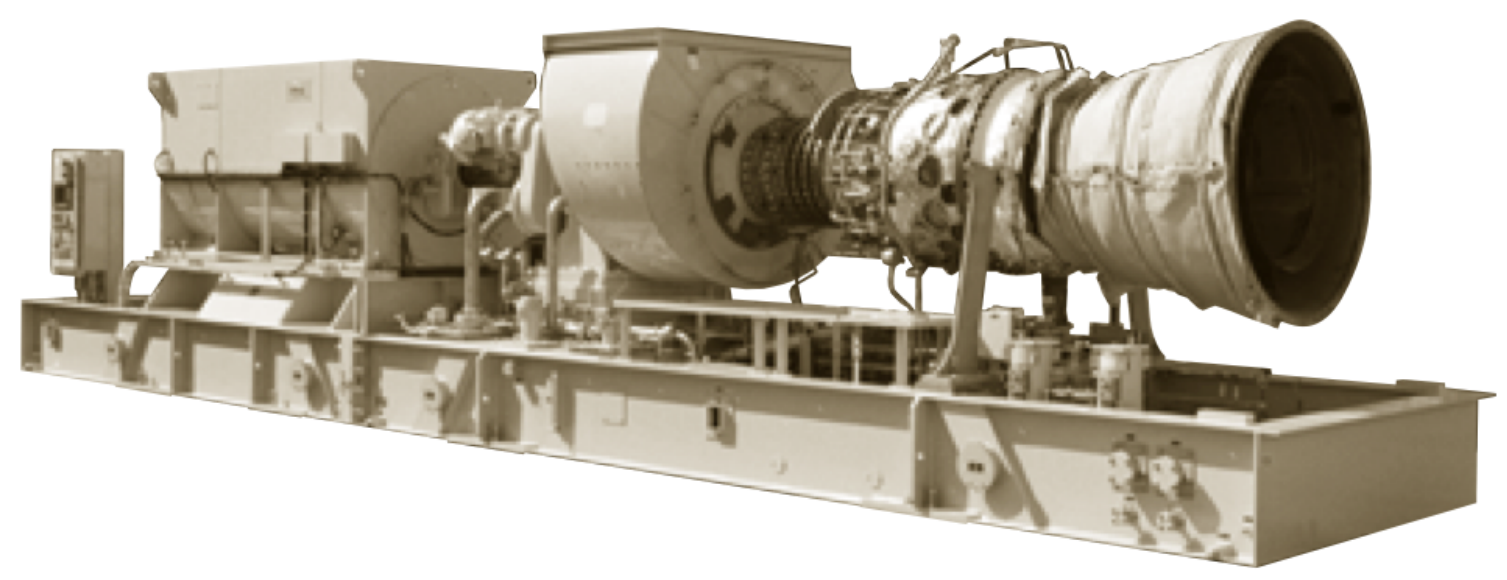

Figure 2: Image of a Solar Turbines Titan 130

(Courtesy Solar Turbines [3] ) 
Aircraft gas turbine engines fall into one of three main classifications; turbojet, turbofan, or turboprop. These gas turbines can vary over a large range of sizes propelling aircrafts as small as a $14 \mathrm{ft}$. wingspan RQ-7 Shadow (military UAV) or as large as a 282 ft. wingspan Airbus A320 (commercial jetliner). A turbojet engine was the first type of gas turbine developed for propulsive use in an aircraft. It consists of a gas generator paired with an inlet and exhaust nozzle. The exhaust gases are expanded through the exhaust nozzle to provide thrust. Turbojet engines have the highest specific thrust, and are the only type of turbine able to be used for sustained supersonic flight through the use of afterburners and inlet cones.

The turbofan engine was conceived in an attempt to improve the propulsive efficiency of the turbojet by reducing the jet velocity exiting the nozzle. Turbofan engines have been developed to operate efficiently at high subsonic speeds and are useful in commercial airliners and military transports. In a turbofan engine a portion of the flow bypasses the gas generator and is ejected through a separate nozzle from the flow that is routed through the gas generator. Typical military aircraft engines have $25-50 \%$ of the flow bypassing the core, while civil aircraft engines have $90-93 \%$. The total thrust provided by a turbofan is a combination of two components; the fan thrust and the thrust from the core of the engine. The reduction of the jet velocity behind the engine was found to have an added benefit of a decrease in the noise production. Figure 3 shows a cutaway view of a GE TF34 turbofan military engine that is used on two subsonic military aircraft, the A-10 Thunderbolt II and S-3 Viking. 


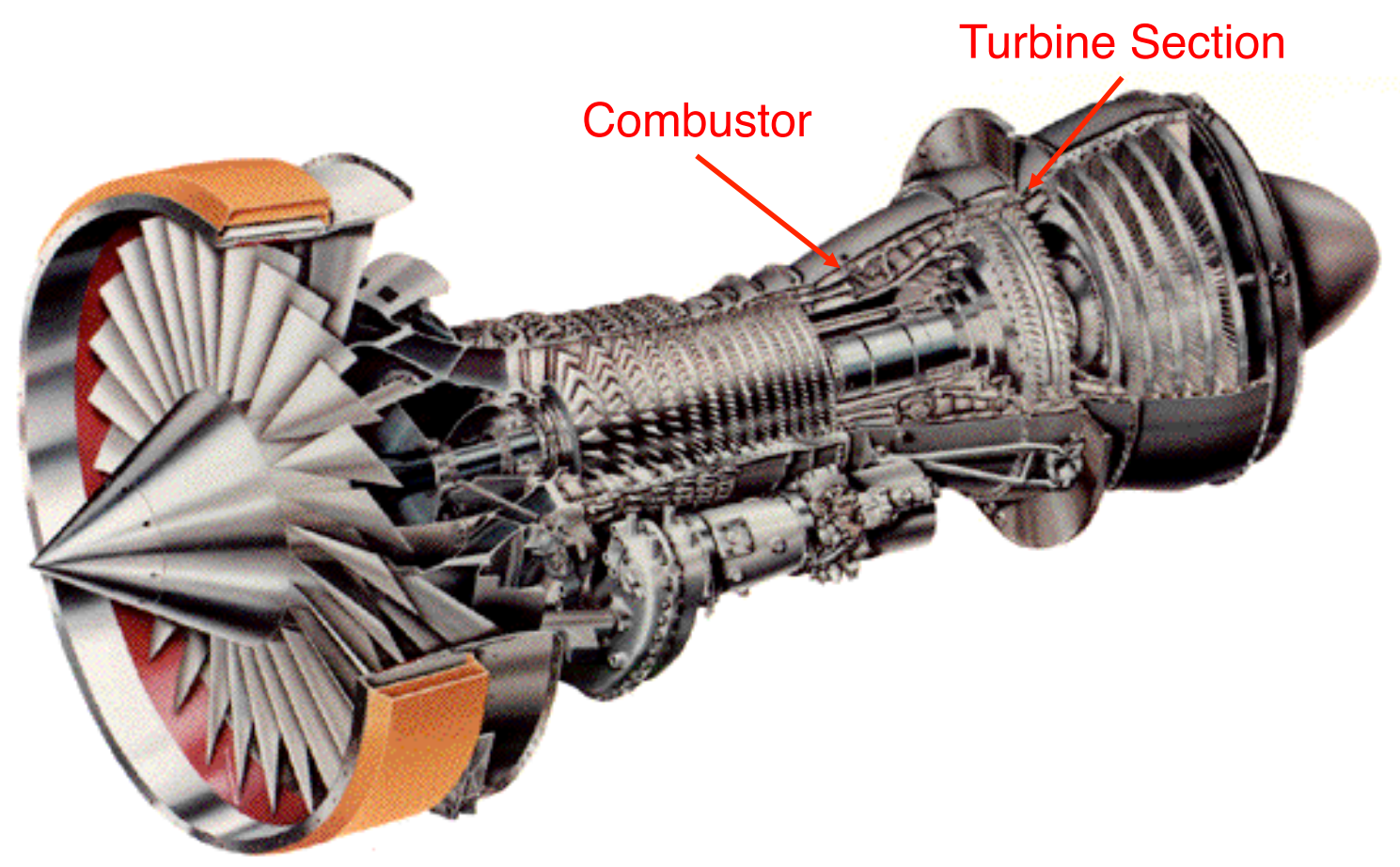

\section{Figure 3: Cutaway View of a GE TF34 9000-Pound Class Turbofan Engine (Courtesy GE [4])}

A turboprop engine is essentially a gas generator connected through a gearbox to a propeller. The shaft power produced by the engine is used to turn a propeller on a plane. Unlike a turbojet or turbofan, a turboshaft engine relies on the turbine extracting as much of the energy as possible to turn the propeller leaving little energy in the exhaust. The majority of the thrust produced by a turboprop is through the propeller and is highly dependent upon the propeller efficiency. A turboshaft engine is similar to that of a turboprop but instead of power being supplied to the propeller of a fixed wing aircraft, the shaft is connected to a gearbox and used to power a helicopter. 


\subsubsection{IDEAL GAS TURBINE CYCLE}

The ideal cycle for a simple gas turbine engine is the Brayton cycle, which is made up of four processes:

1-2 Isentropic Compression Through a Compressor

2-3 Constant Pressure Heat Addition

3-4 Isentropic Expansion Through a Turbine

4-1 Constant Pressure Heat Rejection ${ }^{*}$

A T-s diagram of the ideal Brayton cycle showing the 4 processes is shown in Figure 4.

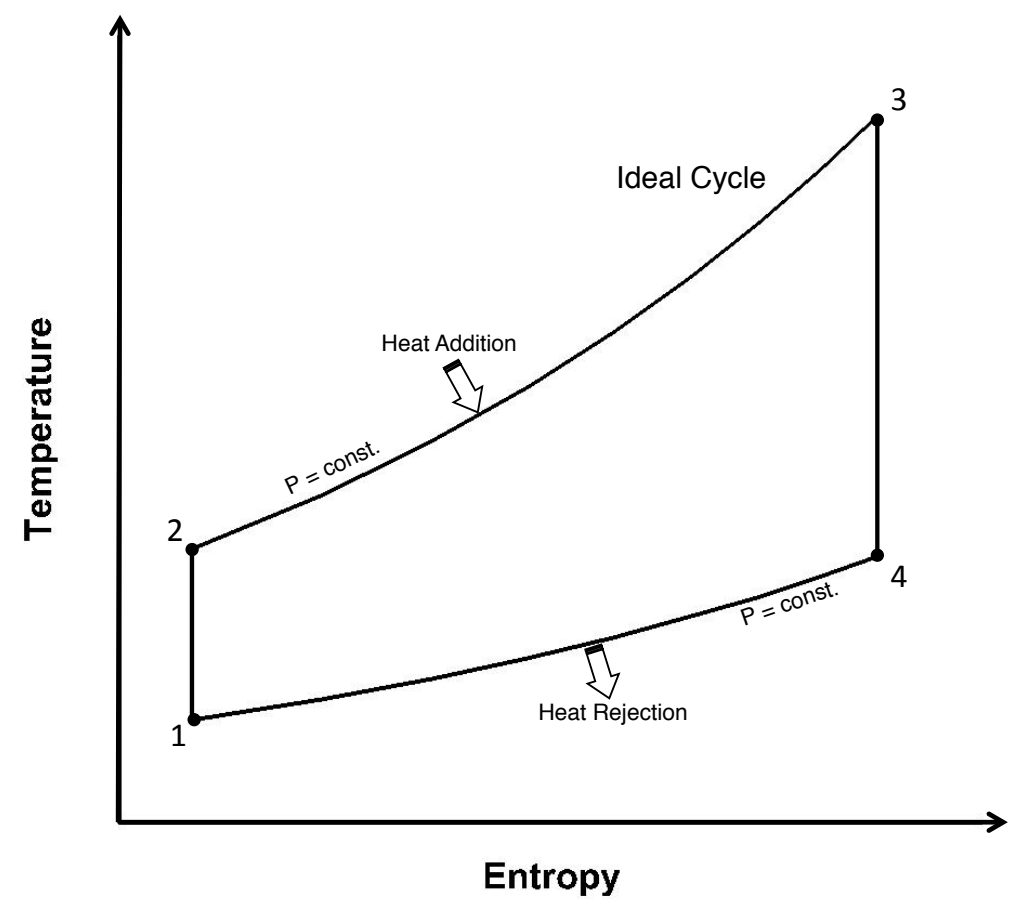

Figure 4: T-S Diagram for Ideal Gas Turbine CyCle

Six main assumptions are needed for an ideal analysis of the Brayton cycle.

1. First, the compressor and turbine are assumed to be isentropic.

2. Next the change in kinetic energy of the working fluid between each section of the turbine is negligible.

*Station 4-1 in a real open engine is the process of exhausting the spent combustion gasses out of the system and allowing the inlet of fresh air into the compressor. 
3. No pressure losses and/or flow leakages through any of the ducts connecting components are present.

4. Air is used as the working fluid and has constant properties throughout the process and any changes in the working fluid through the compression, combustion, or expansion processes are neglected.

5. The mass flow rate through the process is assumed to be a constant and any mass flow of fuel is small enough to omit.

6. The last of the six assumptions is that the cycle is well insulated and all of the heat from combustion or heat addition is used to raise the temperature of the working fluid.

The steady energy balance is shown in equation (1-1).

$$
\begin{array}{r}
\dot{Q}_{\text {in }}-\dot{W}_{\text {in }}+\dot{m}\left(h_{\text {in }}+V_{\text {in }}^{2} / 2+g z_{\text {in }}\right) \\
=\dot{Q}_{\text {out }}-\dot{W}_{\text {out }}+\dot{m}\left(h_{\text {out }}+V_{\text {out }}^{2} / 2+g z_{\text {out }}\right)
\end{array}
$$

Dropping the kinetic and potential energy terms, the result is shown in equation (1-2).

$$
\dot{Q}_{\text {in }}-\dot{W}_{\text {in }}+\dot{m} h_{\text {in }}=\dot{Q}_{\text {out }}-\dot{W}_{\text {out }}+\dot{m} h_{\text {out }}
$$

During the compression process from 1-2 there is no heat addition or rejection nor is there any output work so the work done by the compressor is given in equation (1-3) and is manipulated to show the specific work of the compressor in terms of temperature.

$$
\begin{gathered}
\dot{W}_{\text {in }}=\dot{m}\left(h_{\text {out }}-h_{\text {in }}\right)=\dot{m}\left(h_{2}-h_{1}\right) \\
w_{1-2}=h_{\text {out }}-h_{\text {in }}=h_{2}-h_{1}=c_{P}\left(T_{2}-T_{1}\right)
\end{gathered}
$$

The combustion process from 2-3 has no work done on the system and no heat loss. Equation (1-4) shows the process through the combustor. 
INTRODUCTION

$$
\begin{gathered}
\dot{Q}_{\text {in }}=\dot{m}\left(h_{o u t}-h_{\text {in }}\right)=\dot{m}\left(h_{3}-h_{2}\right) \\
q_{2-3}=h_{o u t}-h_{\text {in }}=h_{3}-h_{2}=c_{P}\left(T_{3}-T_{2}\right)
\end{gathered}
$$

Expansion through the turbine is also assumed to be an adiabatic process as in the case of the compressor with no input work and the heat transfer rate term is neglected. The resulting equation and manipulation is analogous to the compression process and is shown in equation (1-5).

$$
\begin{gathered}
\dot{W}_{\text {out }}=\dot{m}\left(h_{\text {in }}-h_{\text {out }}\right)=\dot{m}\left(h_{3}-h_{4}\right) \\
w_{3-4}=h_{\text {in }}-h_{\text {out }}=h_{3}-h_{4}=c_{P}\left(T_{3}-T_{4}\right)
\end{gathered}
$$

The exhaust process in an open cycle and the heat rejection process in a closed cycle both receive the same treatment in terms of the thermodynamic analysis. Using equation (1-2) and neglecting the work terms and the heat addition term then substituting for the enthalpy as before results in equation (1-6).

$$
\begin{gathered}
\dot{Q}_{\text {out }}=\dot{m}\left(h_{\text {in }}-h_{\text {out }}\right)=\dot{m}\left(h_{4}-h_{1}\right) \\
q_{4-1}=h_{\text {in }}-h_{\text {out }}=h_{4}-h_{1}=c_{P}\left(T_{4}-T_{1}\right)
\end{gathered}
$$

The cycle efficiency for any thermodynamic cycle can be simplified down to what is obtained out of the cycle normalized by what is put into the cycle. In the case of the Brayton cycle for a gas turbine engine the net work out is the difference between total work done by the turbine and work delivered to the compressor by the turbine. The energy put into the system is through heat addition through either combustion or a heat exchanger. This results in the cycle efficiency mathematically shown in equation (1-7).

$$
\eta_{\text {cycle }}=\frac{\dot{W}_{t u r b}-\dot{W}_{c o m p}}{\dot{Q}_{\text {in }}}=\frac{\dot{W}_{\text {net }}}{\dot{Q}_{\text {in }}}=\frac{\dot{Q}_{n e t, c y c l e}}{\dot{Q}_{\text {in }}}=\frac{\dot{Q}_{\text {in }}-\dot{Q}_{\text {out }}}{\dot{Q}_{\text {in }}}=1-\frac{\dot{Q}_{\text {out }}}{\dot{Q}_{\text {in }}}
$$




$$
\eta_{\text {cycle }}=1-\frac{\left(T_{4}-T_{1}\right)}{\left(T_{3}-T_{2}\right)}=1-\frac{T_{1}}{T_{2}} \frac{\left(T_{4} / T_{1}-1\right)}{\left(T_{3} / T_{2}-1\right)}
$$

By rearranging to get the resultant equation (1.7) it can be shown that the cycle has a dependency on the ratio of the turbine inlet temperature to the compressor exit temperature $\left(T_{3} / T_{2}\right)$. By raising the turbine inlet temperature the cycle efficiency increases. This is a fundamental way to increase the overall efficiency of a simple cycle gas turbine, and is a leading reason to improve film cooling performance!

Using the isentropic pressure-temperature relationship it can be shown that the cycle efficiency is more heavily dependent on the pressure ratio. Graphically the relationship between the thermal cycle efficiency and the pressure ratio is shown in Figure 5. The turbine inlet temperature is varied to show the relationship between $T_{3}$ and the compressor pressure ratio.

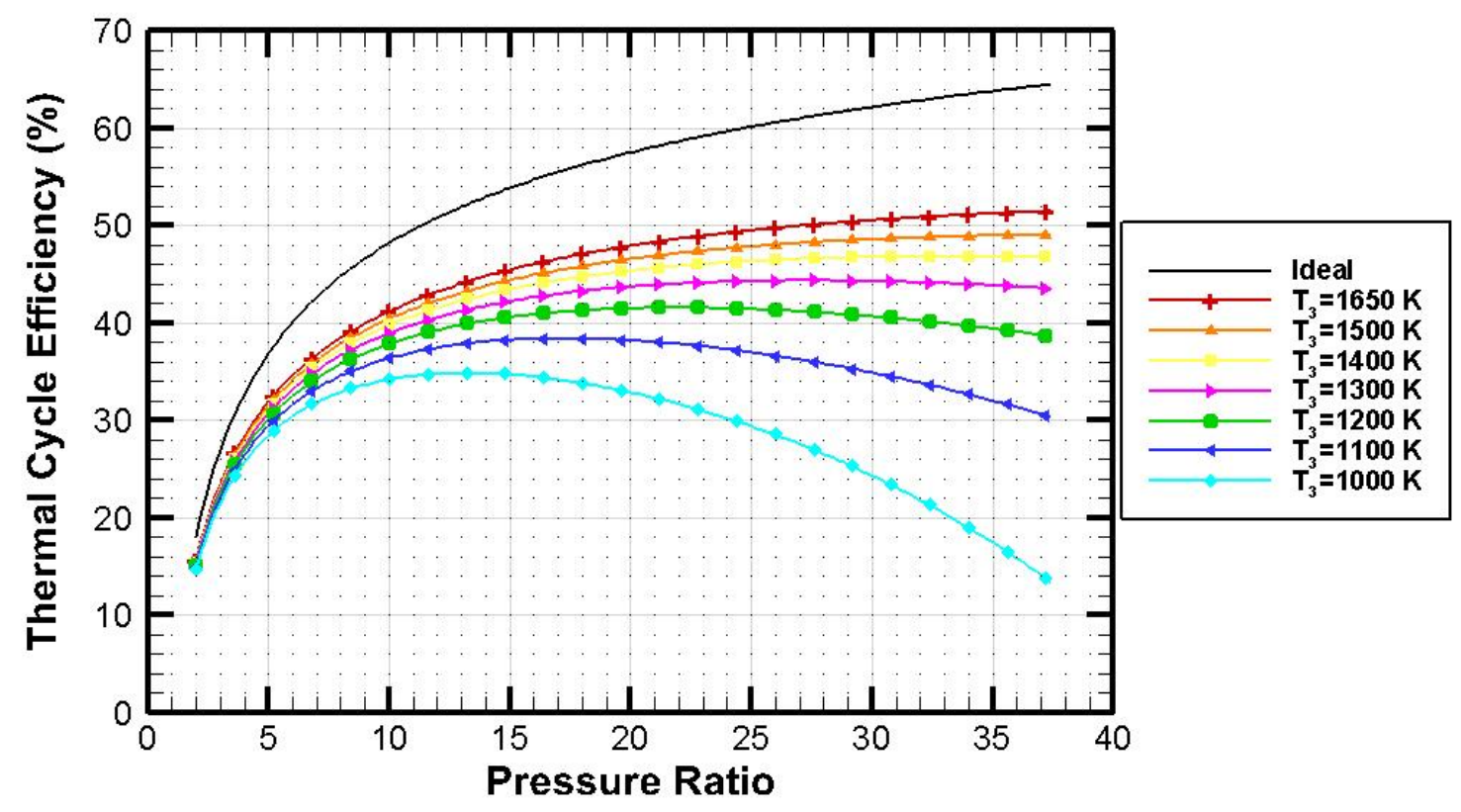

\section{Figure 5: Thermal CyCle EfFiciency of a Simple Gas Turbine Varying With Pressure Ratio ANd Turbine Inlet Temperature}


For a given turbine inlet temperature, there exists an optimal pressure ratio for maximum cycle performance. Increasing the turbine inlet temperature above this pressure ratio would decrease the thermodynamic performance of the engine. It can be seen that as the turbine inlet temperature increase, the optimal pressure ratio increases as well.

The gas turbines of the early $20^{\text {th }}$ century had very low cycle efficiencies, on the order of $15-17 \%$, whereas today's engines can achieve cycle efficiencies over $40 \%$ in simple cycles. Improvements upon the basic cycle to more advanced combined cycles have further increased the efficiencies to over $60 \%$.

Thermal efficiency and power output increase as the turbine rotor inlet temperature increases. Figure 5 shows the thermal cycle efficiency vs pressure ratio. It can be seen that the thermal efficiency increases to a certain point and then starts to decrease. This means that there is a limited pressure ratio for any given temperature and surpassing that temperature or pressure ratio will decrease the system's overall efficiency.

\subsubsection{REAL GAS TURBINE CYCLE}

The ideal gas turbine cycle contains a number of assumptions that cannot be made when trying to predict the performance of a real gas turbine engine. Most importantly for the consideration of this work, the turbine and compressor are not isentropic and the mass flow through the turbine is greater than the mass flow through the compressor. Isentropic (or polytropic) efficiencies are generally defined to take into account these losses through the compressor and the turbine. The deviation from the ideal cycle as a result of theses losses is shown graphically in Figure 6. The further the deviation from the real cycle the lower the thermodynamic cycle efficiency. 


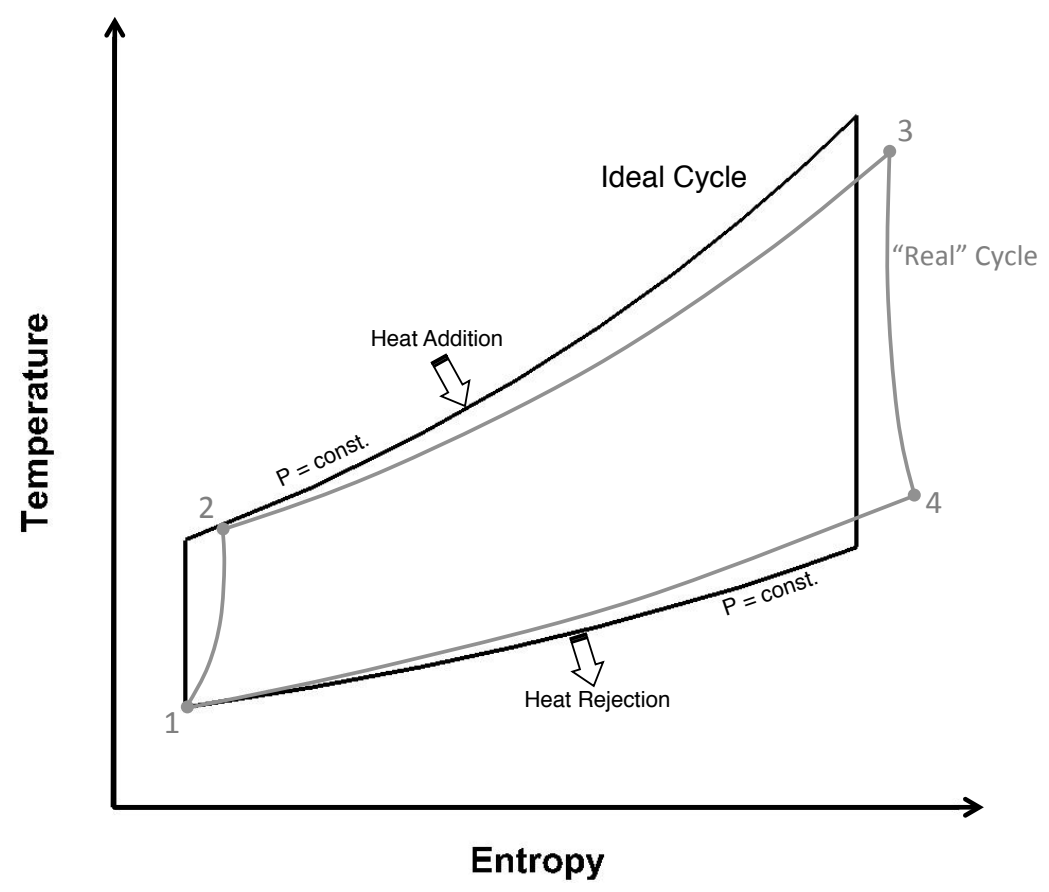

\section{Figure 6: Comparison T-S Diagram of Real ANd Ideal Brayton CyCleS}

For real engines a small amount of air (1-2\%) must be bled off in order to cool turbine discs and blade roots. Modern engines can operate at turbine inlet temperatures greater than $1350 \mathrm{~K}$ resulting in a greater amount of cooling flow to be used in order to internally cool turbine blades. The result may be up to $15 \%$ of the compressor mass flow to be diverted for cooling purposes [1].

The specific work done by the turbine is used to power the compressor (not accounting for shaft losses), and is therefore equal to the specific work done onto the compressor. Additional energy in the flow after the expansion through the high pressure turbine can be expanded further through a low pressure turbine or through a nozzle in an aircraft engine. The mass flow of the bleed air decreases the mass flow through the low pressure turbine and thus the work done by the turbine. To maintain the same net work output the low pressure turbine must produce more specific work. This can be illustrated mathematically in equation (1-8). 


$$
\begin{gathered}
w_{\text {turb }}=w_{\text {comp }} \\
\dot{W}_{\text {net }}=\left(\dot{m}_{a}-\dot{m}_{\text {bleed }}\right) w_{\text {turb }}-\dot{m}_{a} w_{\text {comp }}
\end{gathered}
$$

Efficient use of bleed air from the compressor is important in the isentropic and polytropic efficiency of the turbine. The air from film cooling holes can affect the aerodynamics of the turbine airfoil and degrade the performance. By decreasing the amount of bleed air and maintaining the coolant coverage, the overall efficiency of the engine can be improved.

\subsection{Gas Turbine CoOling And Heat Transfer}

Gas turbines have been improving continuously for the last 80 years driven by the need for improvements in efficiency and power output. Figure 7 shows the development of some historical engines and their improvements over time.

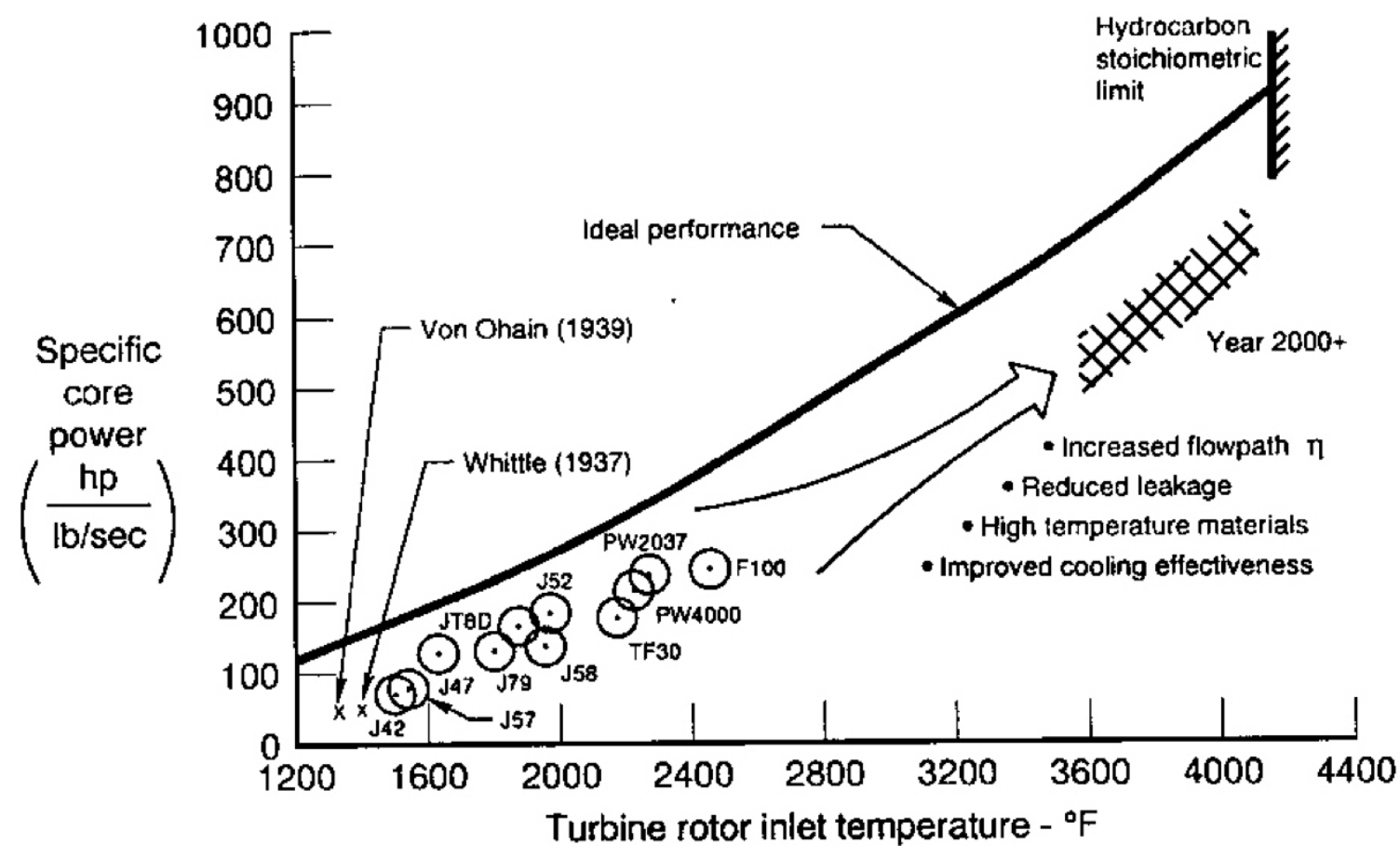

Figure 7: Specific Core Power Production Compared to Turbine Inlet Temperature for Historic Engines and Projections to Future [5] 
Improvements to the overall efficiency and power output of a gas turbine engine are largely driven by the turbine inlet temperature. Modern aircraft gas turbine engines operate at temperatures $(2200-2250 \mathrm{~K})$ above the melting points of the materials in the hot section necessitating that the components be properly cooled. Methods maintaining sufficiently low component temperatures in the hot section of a gas turbine include thermal barrier coatings (TBC) and sophisticated cooling schemes using compressor bleed air. Specific power output is key for aircraft engines, and cooling the hot sections of the engine can allow for hotter, more efficient engines. Land-based, power generation, gas turbines operate at a lower turbine inlet temperature $(1650-1700 \mathrm{~K})$ due to the NOx emissions formed at higher temperatures. These power generation turbines could benefit from the decreased cooling flow bled from the compressor as described in the previous section.

Due to the rotating nature of turbine blades, they generally receive the most attention from a cooling standpoint. Improvements in materials have allowed for safe operation with high thermal loads. A cooled turbine blade allows for a reduction in the thermal load on the blades. Gas turbine blades are cooled both externally and internally. Internal cooling passages utilize the compressor bleed air passed through serpentine passages inside of the blade to remove heat from the surface of the blades in contact with the hot combustion gases. Arrays of cylinders within the serpentine passages promote turbulence and enhance the convective heat transfer from the blade walls; known as pin fin cooling. Ribs or other turbulators may be used in the same way as pin fins to promote turbulence and improve cooling. Another scheme used in the internal passage of the blades is jet impingement of the coolant against the hot walls to enhance the convective heat transfer. 
A significant portion of the coolant passed through the internal passages of the blades is ejected at discrete locations on the blade into the main stream flow in order to provide a cooling layer near to the surfaces of the blades. This external cooling is synonymous with film cooling when discussing cooling of turbine blades. Figure 8 shows the rudimentary development of a cooled turbine blade. The left most blade is cooled solely through internal passages. As you move to the right the blades are cooled by more complex serpentine passages and there is more extensive film cooling with better coverage. The far right blade schematically represents a modern cooling design circa 1980s and is currently in use today.

L.P. cooling air

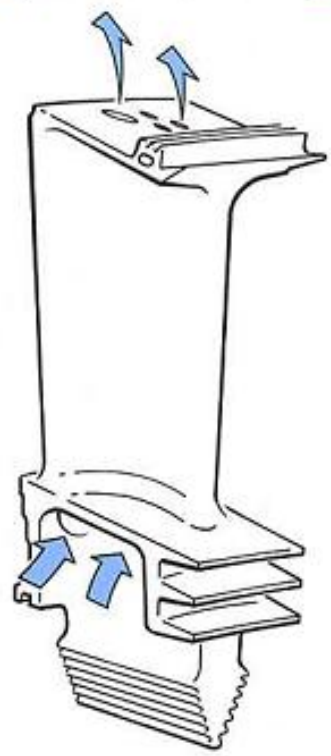

SINGLE PASS INTERNAL COOLING (1960's)
H.P. cooling air

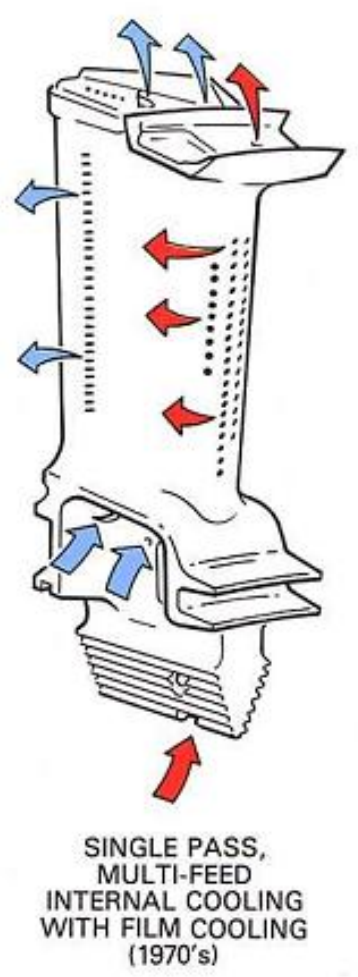

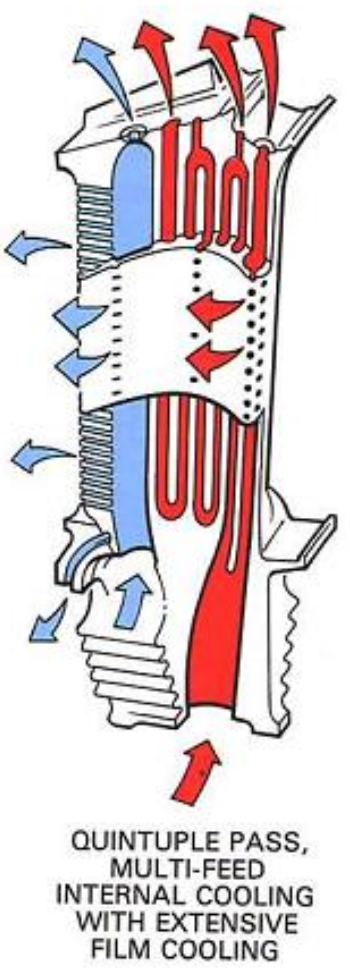

Figure 8: Diagram the Development of a Cooled Turbine Blade [6] 
It is critical for safe and efficient operation of gas turbines that cooling schemes are well studied and implemented. Predictable heat transfer to the components is essential in determining the operating limits of an engine.

Film cooling by definition is injecting a cooler flow under the boundary layer to protect a surface from the high heat transfer from the hot gasses of the free stream. In gas turbine applications there has been a great deal of research and development since the 1970s and 1980s when film cooling was first being implemented in actual engines. Variations of the size, spacing, geometry, and injection angle have been well researched in the past 30 years. Section 1.5 will cover the most important aspects of film cooling as they apply to this study. 


\subsection{TuRbulence}

Although laminar and inviscid flows can be good approximations for a number of "real" flows in nature most flows deviate from these assumption and are turbulent. Basically, as flow Reynolds number increases, a base laminar flow state becomes unstable to small forces or perturbations and undergoes a transition from laminar to turbulent flow. Through a simple manipulation of the Reynolds number it can be shown that the Reynolds number can be thought of as the ratio of the inertial forces to the viscous forces as shown in equation (1-9).

$$
\begin{gathered}
R e=\frac{\rho U L}{\mu}=\frac{U L}{v} \\
\frac{\rho U L}{\mu} \cdot \frac{U L}{U L}=\frac{\rho U^{2} L^{2}}{\mu \frac{U}{L} L^{2}} \propto \frac{q_{\infty} S}{\tau_{w} S}=\frac{F_{\text {inertial }}}{F_{\text {viscous }}}
\end{gathered}
$$

Therefore, as the Reynolds number increases $\left(R e>>1\right.$ and $\left.F_{\text {inertial }} \gg F_{\text {viscous }}\right)$ the inertial forces become dominant and the viscous forces become too small to damp out disturbances. Turbulence is especially important in the flow through a gas turbine engine. The rotating nature of the components, complex geometries, and high Reynolds numbers involved create a highly turbulent environment.

\subsubsection{CHARACTERIZATION OF TURBULENCE}

Turbulence in itself is a widely studied area of fluid mechanics and, although much progress has been made in the last fifty years, much more study is needed to fully understand turbulence. It is impossible to accurately describe turbulence with one equation or characteristic. Mathieu and Scott [7] describe 11 primary characteristics that 
can help better define the phenomenon of turbulence. These characteristics are described within this section.

I. Turbulence appears random and chaotic in both space and time. This can be seen by looking at any velocity data at any fixed position versus time. The signal will show that the velocity fluctuates due to turbulent eddying motions, both large and small. Figure 9 shows a velocity signal produced in the experimental film cooling wind tunnel at WVU. The large scale eddy motions have smaller scale motions superimposed on them. This can be seen in the fluctuating velocity data by magnifying the data and looking more closely at the signal as is shown in the bottom half of Figure 9. Further magnification would be able to show fluctuations all the way down to the smallest length scale if aliasing was not present in the data at that level of magnification.

II. Turbulence is comprised a continuum of infinitely many length and time scales. The largest eddy size is known as the integral length scale or large eddy length scale $(\Lambda)$. There exist progressively smaller length scales in a cascade of energy from the large eddy length scale all the way down to the smallest eddy size. The smallest of the turbulent eddies is know as the Kolmogorov length scale $\left(\eta_{\mathrm{k}}\right)$. The "fuzziness" of the velocity signal, as shown in Figure 9, is due to the superposition of the effects of all of the length scales on the velocity. The large scale fluctuations are evident in the fluctuations of the flow and the smaller scales are what provided the "fuzziness" to the signal. 

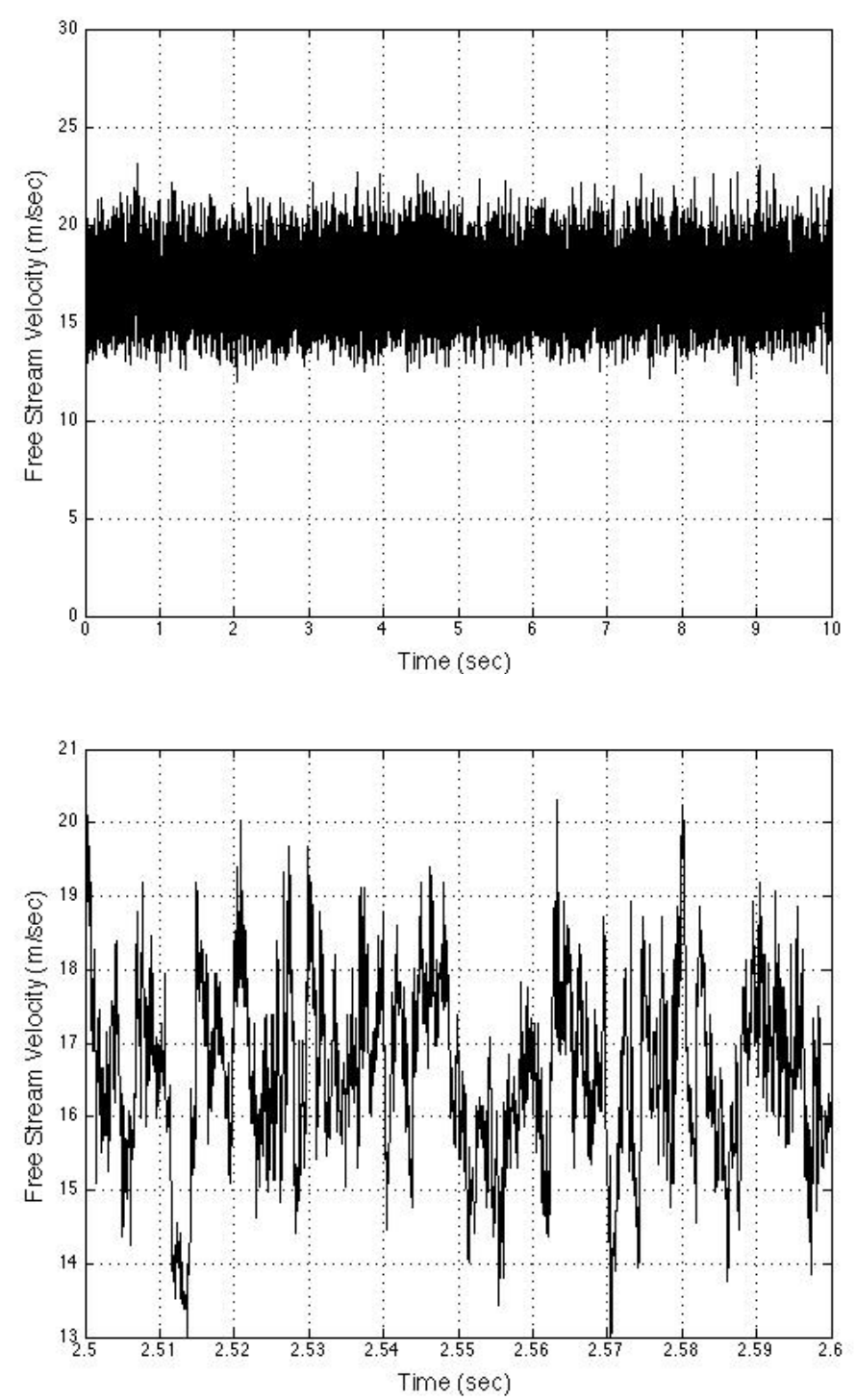

Figure 9: Turbulent Velocity FluCtuAtion

III. Turbulence contains small-scale random vorticity. A turbulent flow by definition has vorticity and at the smallest of the length scales is the cause of the small-scale vorticity that is random in both space and time.

IV. Turbulence occurs at "high" Reynolds number. As mentioned before, increasing the Reynolds number increases the ratio of the inertial forces to the viscous forces. Flow instabilities become too great for the viscous terms to damp out. 
V. Turbulence dissipates energy. At the largest scales of turbulence kinetic energy is conserved and smaller scale eddies extract energy from the larger scale eddies in a cascade of energy all the way down to the Kolmogorov scale. The largest eddies are anisotropic, and can affect the energetics of the mean flow. The Kolmogorov scale eddies exist at high frequency, and are nearly isotropic and homogenous.

VI. Turbulence is a continuum phenomenon. The small length scales are many orders of magnitude smaller than the integral length scale.

VII. Turbulence is a 3-D phenomenon. Numerical studies of two dimensional turbulence have shown that small scale eddies combine to form larger ones, in direct contrast to the physical cascade of energy from the large to small scales.

VIII. Turbulence display large eddy scales that become independent of turbulent Reynolds number. At these high Reynolds numbers the large scale eddies are nearly inviscid and are insensitive to the changing Reynolds number. Properties of the turbulent flow affected by the large scale eddies are essentially independent of the Reynolds number as well.

IX. Turbulence is often intermittent. The intermittency can be as seemingly random as the velocity signals. Figure 10 shows a schematic of an intermittent flow.

X. Turbulence displays or has smallest eddy scales, becoming nearly isotropic/homogenous/independent of the mean flow strain rate.

XI. Turbulence is inherently a non-linear process. It is due to the instabilities in the convective acceleration terms of the Navier-Stokes equations $\left(U_{j} \frac{\partial U_{i}}{\partial x_{j}}\right)$. 


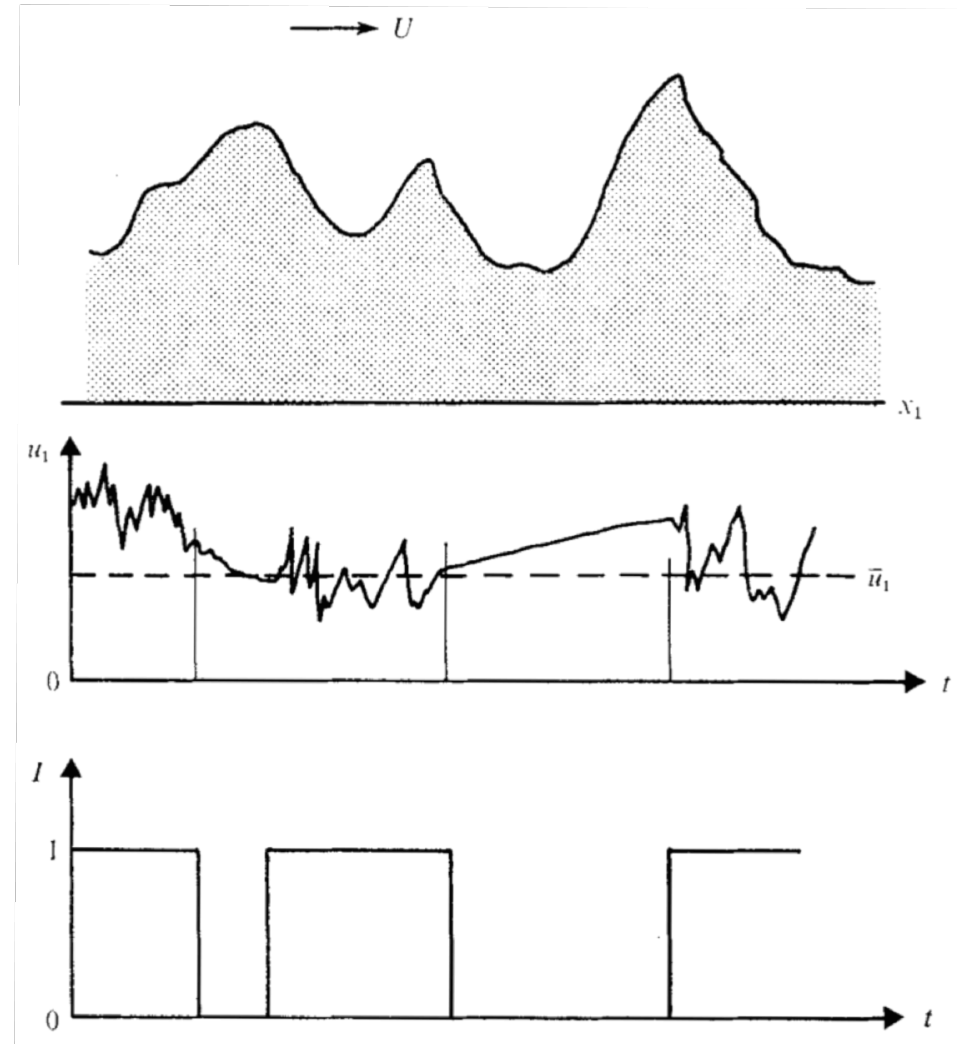

\section{Figure 10: SCHEMATIC REPRESENTATION Of AN INTERMitTENT Flow[8]}

\subsubsection{SIGNAL DECOMPOSITION, TURBULENCE INTENSITY AND LENGTH SCALE}

Methods of statistically averaging turbulent flows were introduced by Reynolds [9] in the late 1800 and are the basis of much of the current theory of turbulence. A velocity signal at a single point can be decomposed into a combination of the mean flow velocity and the fluctuating component as shown in equation (1-10). This is known as Reynolds decomposition and is a crucial step in the derivation of the Reynolds Averaged Navier Stokes (RANS) equations used for computational fluid dynamics (CFD).

$$
U=\bar{U}+u^{\prime}
$$

Figure 11 shows a visual depiction of the Reynolds decomposition for a generic velocity signal. 


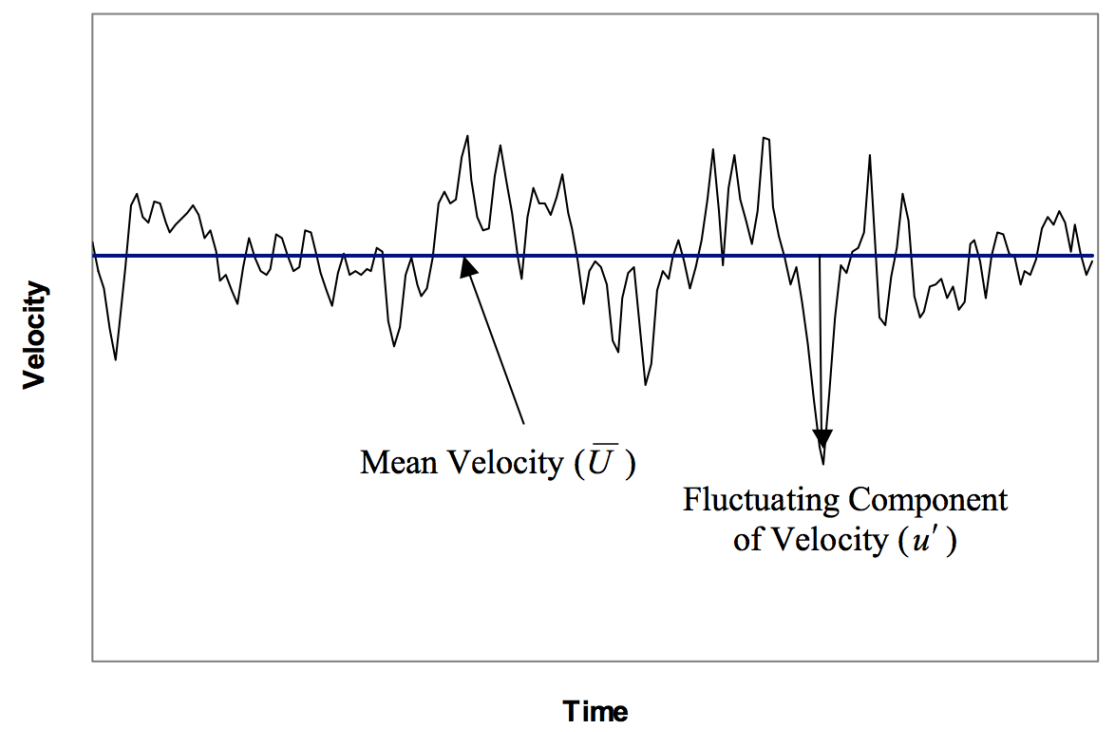

Figure 11: Reynolds Decomposition of TuRbulent Velocity Signal [10]

Turbulence intensity is determined from the decomposition of the velocity signal using the mean and fluctuating components as shown in equation (1-11).

$$
T u=\frac{\sqrt{\overline{u^{\prime 2}}}}{\bar{U}}=\frac{u_{R M S}^{\prime}}{\bar{U}}
$$

The root mean square (RMS) value of the fluctuating velocity is determined by squaring the fluctuating component at every instant, time averaging the squares, and then taking the square root. The turbulence intensity is shown mathematically for a discrete signal in equation (1-12).

$$
u_{R M S}^{\prime}=\sqrt{\frac{1}{N} \sum_{i=1}^{N} u_{i}^{\prime 2}}
$$

The turbulence intensity can be determined in the frequency domain instead of the time domain as shown above. This is accomplished through the use to the power spectral density of the fluctuating velocity signal. The length scale of the turbulent flow can be 
computed through an autocorrelation of the fluctuating velocity component. Details on calculating the turbulence intensity and length scale can be found in [10] or a textbook on turbulence.

\subsection{Overview of CurRent Work and Problem Statement}

The goal of any film cooling study is to reduce the amount of cooling flow needed while more efficiently using said cooling flow. By doing this the performance of a gas turbine engine can be improved for reasons explained in section 1.1.2. Previous researchers [11-13] developed a novel film cooling geometry, an anti-vortex hole (AVH), and were able to show that the AVH concept can mitigate or counter the vorticity generated by conventional holes and increase cooling effectiveness at high blowing ratios and low turbulence levels. Due to the fact that the mechanism for the effectiveness of the AVH is through interaction of vortical structures from the main and side film cooling holes and the potential of a counter rotating vortex (CRV), the effect that the combustor exit turbulence will have on the capacity of the AVH to effectively cool the surface of an airfoil is of concern. The goal of the present study is to attempt to discern the effects of turbulence on the performance of the AVH by varying the turbulence intensity and length scale to be more representative of engine conditions than the previously studied low turbulence case. A high blowing ratio of 2.0 is considered in this study. The phenomenon of the coolant jet detaching and lifting away from the surface leading to the formation of the $\mathrm{CRV}$ is only problematic at high blowing ratios. The AVH concept is best utilized in the regime and would not have much benefit over cylindrical holes that do not have jet lift off. 
The current work begins with nine adiabatic film cooling cases for the studied AVH geometry with turbulence intensities of 5, 10 and $20 \%$ and normalized length scales based on film cooling hole diameter of 1,3 and 6 . The nine adiabatic cases are then replicated with a specified wall heat flux for calculation of heat transfer coefficients, as shown in Table 1.

TABle 1: Test MATRiX

\begin{tabular}{|c|c|c|c|c|}
\hline Case Number & Turbulence Intensity $(\mathrm{Tu})$ & Length Scale $\left(\Lambda_{\mathrm{x}} / \mathrm{d}_{\mathrm{m}}\right)$ & Wall Condition & Solver \\
\hline 1 & 5 & 1 & Adiabatic & RANS \& URANS \\
\hline 2 & 10 & 1 & Adiabatic & RANS \& URANS \\
\hline 3 & 20 & 1 & Adiabatic & RANS \& URANS \\
\hline 4 & 5 & 3 & Adiabatic & RANS \\
\hline 5 & 10 & 3 & Adiabatic & RANS \\
\hline 6 & 20 & 3 & Adiabatic & RANS \\
\hline 7 & 5 & 6 & Adiabatic & RANS \\
\hline 8 & 10 & 6 & Adiabatic & RANS \\
\hline 9 & 20 & 6 & Adiabatic & RANS \\
\hline 1 & 5 & 1 & Specified Heat Flux & RANS \\
\hline 2 & 10 & 1 & Specified Heat Flux & RANS \\
\hline 3 & 20 & 1 & Specified Heat Flux & RANS \\
\hline 4 & 5 & 3 & Specified Heat Flux & RANS \\
\hline 5 & 10 & 3 & Specified Heat Flux & RANS \\
\hline 6 & 20 & 3 & Specified Heat Flux & RANS \\
\hline 7 & 5 & 6 & Specified Heat Flux & RANS \\
\hline 8 & 10 & 6 & Specified Heat Flux & RANS \\
\hline 9 & 20 & 6 & & \\
\hline
\end{tabular}

Since a film cooling flow in a turbulent flow field is an inherently unsteady phenomenon, an unsteady analysis is needed to fully discern the effects of turbulence on the AVH. However, limits in computational resources restricted the present unsteady analysis to an unsteady RANS (URANS) analysis as opposed to more accurate LES or DNS studies. Results from Repko et al. [14] showed that length scale had little effect on the cooling effectiveness of the AVH. This result allowed for a reduction in the number of unsteady cases from nine to three, looking solely at the effect of turbulence intensity on the cooling performance of the AVH. Cases 1-3 were examined using an unsteady formulation. 


\subsection{ReleVAnt Past StUdies}

Since the thermal efficiency and power output of a gas turbine increase as the turbine inlet temperature increases; a great deal of work has been done to improve the cooling of the turbine. Increasing the temperature of components in the hot section of a gas turbine by $10-20 \mathrm{~K}$ can effectively halve the operational life of a component [15]. Increasing the thermal efficiency or increasing the durability is a major tradeoff in gas turbine design.

Although internal cooling schemes exist and are used in industry, they are not considered relevant to the current work. Han, Dutta, and Ekkad [16] give an in depth review of past literature and on internal film cooling spanning more than 100 pages. The concept of film cooling has been studied for over 70 years to protect a generic surface from a hot freestream. Film cooling is the current focus of much research and has been employed in gas turbines since the 1980s [1], and has been effective in reducing the thermal load on components within the engine. Current cooling schemes can use anywhere from $1.5-5 \%$ of the air mass flow, and can reduce blade temperatures by 200 $300 \mathrm{~K}[1,16]$.

Film cooling research for a flat plate geometry is common and assumes that the curvature of the blade is negligible. Past studies have proved the validity of this assumption, and that flat plate models can be applied to real engine applications with minor corrections [16]. It has long been known that the key parameter in film cooling is the blowing ratio $(B R)$ defined in equation (1-13) [17]. The blowing ratio is essentially the ratio of the mass fluxes of the coolant and the hot freestream gases. Some other key parameters to consider when comparing film cooling performance are the density ratio 
$(D R)$ of the freestream to coolant and the Reynolds number based on film cooling hole diameter $\left(R e_{d}\right)$. These parameters are defined in equations (1-14) and (1-15) respectively.

$$
\begin{gathered}
B R=\frac{(\rho U)_{c}}{(\rho U)_{\infty}} \\
D R=\frac{\rho_{c}}{\rho_{\infty}} \\
R e_{d}=\frac{\rho_{\infty} U_{\infty} d_{m}}{\mu}
\end{gathered}
$$

\subsubsection{FILM COOLING}

For a low speed, constant property flow, a dimensionless adiabatic wall temperature is defined as $\eta_{\text {aw }}$, shown in equation (1-16). This dimensionless adiabatic wall temperature is well known as the adiabatic film cooling effectiveness. A large number of past studies in film cooling have looked almost exclusively at the adiabatic film cooling effectiveness when evaluating a film cooling flow.

$$
\eta_{a w}=\frac{T_{\infty}-T_{a w}}{T_{\infty}-T_{c}}
$$

When the blowing ratio for a convectional straight film cooling hole becomes sufficiently high the cooling flow may detach from the surface in a phenomenon referred to as jet lift off. The coolant jet lift off generally occurs at blowing ratios greater than 0.5 [13]. Blowing ratios greater than approximately 1.5 are considered high and are often seen in practice with real engines. When jet lift off occurs a CRV pair is created. The CRV pulls hot gases from the freestream and entrains them near to the wall increasing the thermal load on the component being cooled. Haven et al. [18] produced a diagram depicting the CRV pair and hot gas entrainment shown in Figure 12. The vorticity 
generated stems from shear interaction between the hot mainstream flow and the coolant jet as well as a secondary interaction with the solid wall. Near to the injection hole the coolant has enough momentum to keep its shape and acts similar to a solid cylinder, with the mainstream gases flowing around it as is the case for a cylinder in a cross flow.

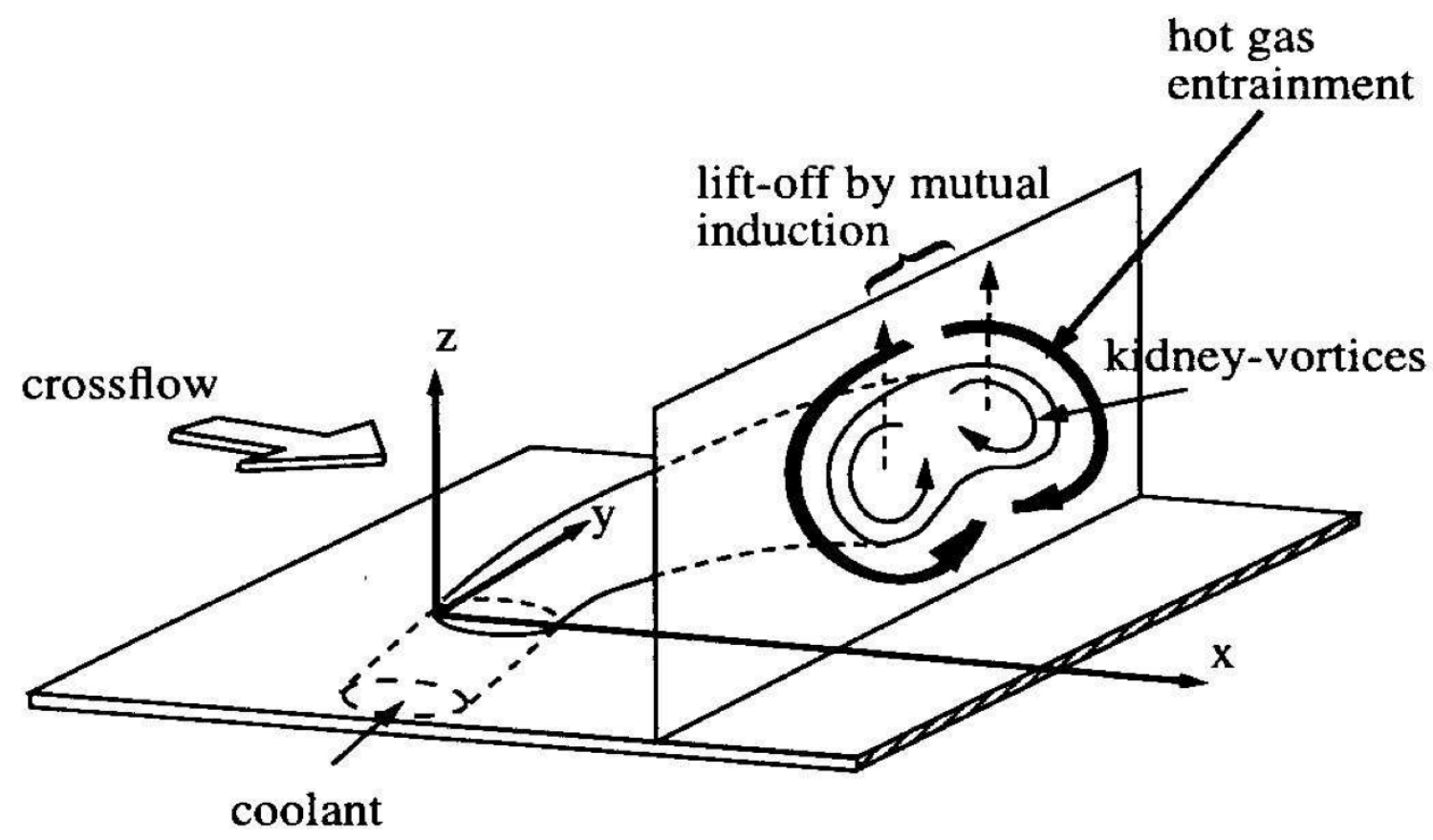

\section{Figure 12: Diagram Depicting The Counter Rotating Vortex (CRV) Problem (Haven et al. [18])}

Density ratio is generally around 2.0 in a modern gas turbine engine. The effect of density ratio is dependent upon the blowing ratio. Ammari et al. [19] showed that the effect of density ratio on the heat transfer coefficient contours downstream of a film cooling hole is minimal at low blowing ratios. Increasing blowing ratio showed that there can be a substantial effect, especially on jet lift off, as the density and blowing ratio approach representative engine conditions. The two density ratios studied were 1.0 and 1.52. At the larger of the two density ratios and at the highest blowing ratio $(\mathrm{BR}=2.0)$, a substantial change in the jet liftoff and reattachment was noted. 
Numerous groups have developed novel film cooling geometries aimed at reducing the cooling flow and limiting jet lift off. One of the most commonly used techniques that has had success at combating the jet lift off is the use of shaped film cooling holes. Bunker [20] provides a review of shaped film cooling technology from its inception to 2005 concluding that the target for shaped film cooling holes is to "expand the exit area in the plane of the surface of injection jet by a factor or 2-3 times that of the round jet without separation." Expanding the area of the cooling hole decreases the momentum of the cooling jet promoting an attached film. Figure 13 includes a fan shaped hole that diffuses the coolant allowing for better attachment and better coverage of the coolant jet.

An innovative concept aimed to reduce the CRV without changing the geometry of the cooling hole was proposed by Rigby and Heidmann [21]. They proposed placing vortex generator downstream of the cooling hole in order to create vortices to lessen the effect of the CRV. Although the results appeared viable, machining the vortex generator and cooling the surfaces that protrude into the main flow would be problematic.

Several studies have showed placing cylindrical holes in a trench increases the performance over the same cylindrical holes not in a trench. Zuniga et al. [22] found that the same cooling performance, for a given number of cylindrical cooling holes, can be achieved by doubling the distance between the holes and trenching them. This study was also able to show that the benefits of trenched cooling holes are not advantageous for shaped holes. Shaped cooling holes have their performance degraded when placed in a trench. 


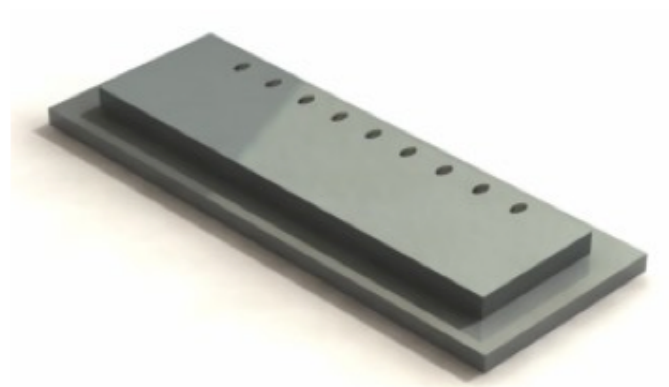

Cylindrical Holes

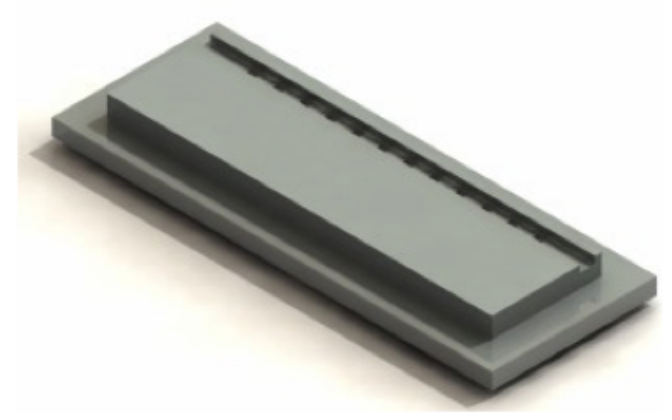

Trenched Holes
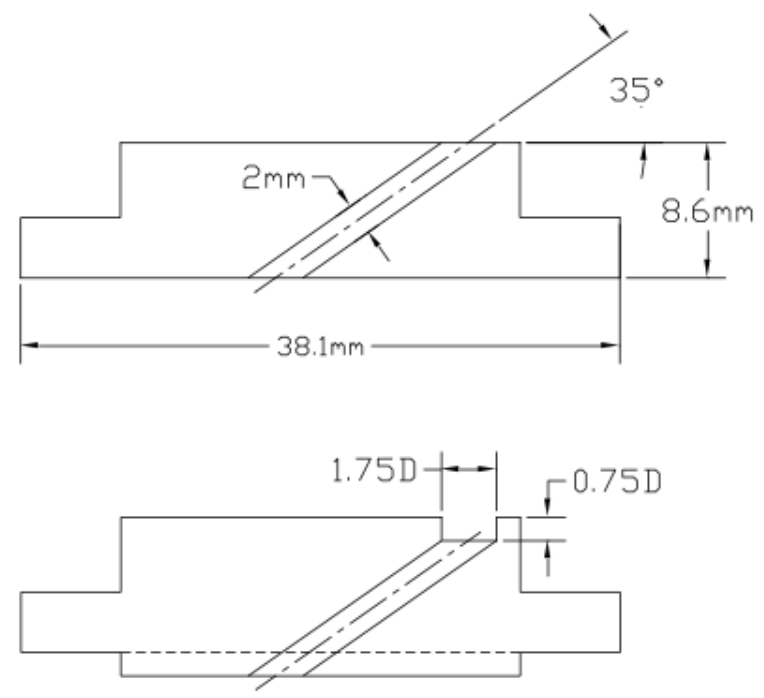

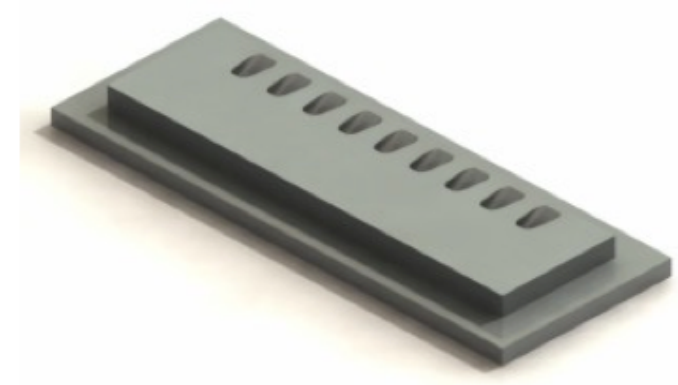

Shaped Holes

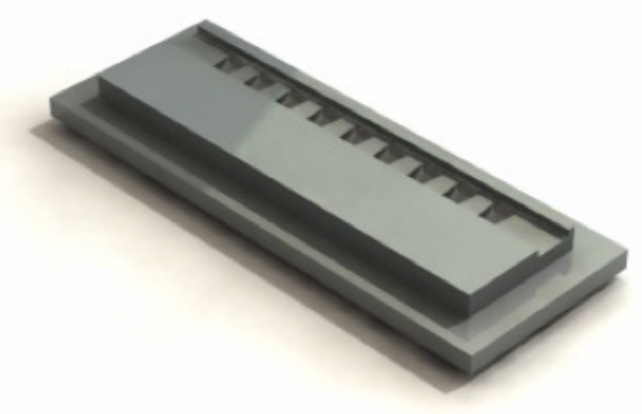

Trenched Shaped Holes
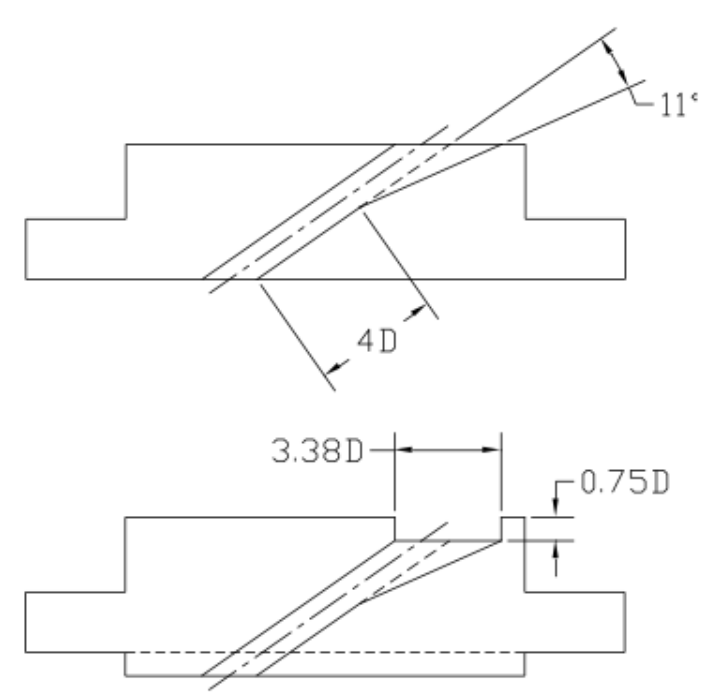

\section{Figure 13: Coupons Showing Geometries of VARious Film Cooling GEOMETRIES [22]}

The notion of "sister holes" was introduced by Ely et al. [23]. Sister holes are of a similar idea to the AVH in that they use additional cooling jets stemming from side holes to counter the vorticity generated by the main cooling hole. The sister hole design uses two separate cooling holes flanking the main cooling hole instead of two holes sharing 
the same attachment to the plenum, as is the case with the AVH. The exit of the sister holes into the freestream flow is in a similar location to the main hole compared to the AVH. It was shown that there is a considerable cooling advantage over the cylindrical holes across the cooling domain extending 30 hole diameters down stream.

Heidmann et al. [13] developed the anti-vortex concept, which could possibly, through optimization, reduce or cancel the vorticity of the CRV pair, and not just lessen its effect as in shaped holes. Differing from the sister holes concept, the AVH has its secondary holes intersect the main hole allowing one inlet to feed the coolant to all of the holes as discussed earlier. A beneficial effect of this design is to slightly diffuse the coolant flow and allow it to stay attached for higher plenum pressures (blowing ratios). It is intended that the side holes interact with one another when in a row to produce a strong upwash, which must be balanced by a net downwash in the main hole jet centerline plane.

The current study is concerned with investigation of the effectiveness of the AVH in the presence of elevated levels of turbulence. The geometry of the AVH used in the current study is given in Figure 14 and Table 2 and is consistent with the tested geometry from previous studies [11-14, 24]. 


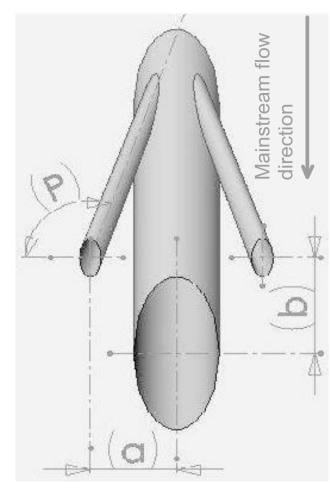

Top View

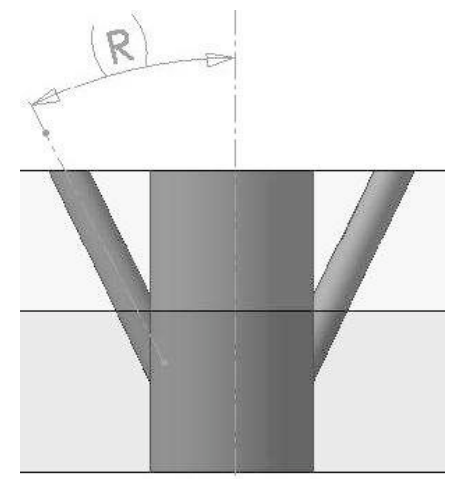

Front View

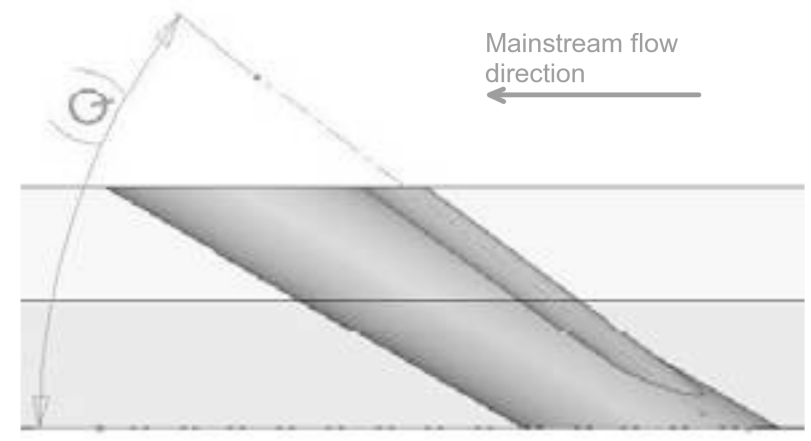

Side View

Figure 14: Three View Drawing of Anti-Vortex Hole (AVH)

\section{Table 2: Geometric Parameters For Tested Version AVH}

\begin{tabular}{|c|c|}
\hline \hline $\mathrm{d}_{\mathrm{s}} / \mathrm{d}_{\mathrm{m}}$ & 0.5 \\
\hline $\mathrm{a} / \mathrm{d}_{\mathrm{m}}$ & 1.12 \\
\hline $\mathrm{b} / \mathrm{d}_{\mathrm{m}}$ & -0.44 \\
\hline $\mathrm{P}$ & $105.67^{\circ}$ \\
\hline $\mathrm{Q}$ & $26.41^{\circ}$ \\
\hline $\mathrm{R}$ & $27.91^{\circ}$ \\
\hline
\end{tabular}

Concurrent to this research, LeBlanc et al. [25] continued to develop the AVH concept and changed the geometry so that the side holes were of the same diameter of the main hole. Also included in the study was a trenched AVH design that showed improvements in the trench but reduced effectiveness further downstream. This AVH design used $50 \%$ less coolant than cylindrical holes with a $30 \%-40 \%$ increase in overall averaged effectiveness. 


\subsubsection{ENGINE CONDITIONS AND EFFECTS OF TURBULENCE}

Most past film cooling studies, both numerical and experimental, have considered only low freestream turbulence conditions. Heidmann et al. [12, 13] also only looked at low turbulence $(\sim 1 \%)$ in the freestream when developing the AVH. Due to the fact that the mechanism for the effectiveness of the AVH is through interaction of vortical structures from the main film cooling and side cooling holes and the potential CRV, the effect of high-intensity, large scale combustor exit free stream turbulence will have on the capability of the AVH to effectively cool airfoil surfaces is of concern. The hot gas flow leaving the combustor is not well characterized but is known to be highly turbulent. This highly turbulent flow increases the heat transfer to the hot section components, especially in the first stage of a gas turbine, and can lead to thermal failure of the components. Previous studies by Van Fossen and Bunker [26] have shown that turbulence intensities can be as high as $20-30 \%$ in a simulated engine environment which measured the intensity and scale of turbulence downstream of a GE90 combustor segment with cold, pressurized flow. Studies by Wang et al. [27] and Barringer et al. [28] have modeled the combustor exit turbulence in the same intensity range with length scale to blade chord length $\left(\Lambda_{\mathrm{x}} / \mathrm{c}\right)$ in the range of 0.11-0.43. Van Fossen and Bunker [26] and Nix et al. [10] showed that a realistic length scale to blade chord diameter is on the order of 0.3 . This scale normalized by the film cooling hole diameter would be approximately 10-15 in modern aircraft engines. The current work focuses on length scales based on film cooling hole diameter between 1 and 6 due to limitations in the maximum length scale of turbulence that can be generated in the current facility. 
The characteristics of the turbulent flow exiting the combustor are not easily characterized, and can vary widely depending on combustor geometry and operational conditions. Past studies, more numerous than can be covered here, have been performed to investigate the effect that elevated levels of freestream turbulence has on film cooling hole geometries. Bons et al. [29] found that high freestream turbulence can decrease film cooling effectiveness for baseline cylindrical cooling holes along the hole centerline, but increase the effectiveness along the midline between holes. Saumweber et al. [30] showed that the effect of turbulence on shaped film cooling holes is detrimental at all blowing ratios, yet cylindrical cooling holes experience slight gains in effectiveness at high blowing ratios. The findings from this study were reiterated in [31] for the cylindrical case and it was found that fan-shaped cooling holes also have their performance degraded by increasing the freestream turbulence. 


\section{COMPUTATIONAL AND EXPERIMENTAL SETUPS}

Computational fluid dynamics (CFD) is a useful tool when analyzing systems involving fluid flow and heat transfer. Reduced down to its most basic form, CFD discretizes and solves the partial differential equations that govern the physics in the continuum being analyzed. Much like experimental methods, the degree of accuracy is directly related to the amount of time and capital allotted to perform the analysis. For simple laminar flows the continuity and Navier-Stokes equations can be simplified and solved analytically. The incompressible Navier-Stokes equations in conservation form are given in equations (2-1) and (2-2).

$$
\begin{gathered}
\frac{\partial U_{i}}{\partial x_{i}}=0 \\
\frac{\partial U_{i}}{\partial t}+U_{j} \frac{\partial U_{i}}{\partial x_{j}}=-\frac{1}{\rho} \frac{\partial P}{\partial x_{i}}+v \frac{\partial U_{i}}{\partial x_{j} x_{j}}
\end{gathered}
$$

Although useful, analytical solutions are not universally applicable to the complex turbulent flows that are of interest. Essentially three treatments of turbulence exist to analyze fluid flow with CFD. The first is a RANS solver, and is the least computationally expensive. RANS relies heavily on empirically derived turbulence models to close the 
system of equations being solved leading to it's limited accuracy. Large eddy simulations (LES) use spatial filtering to separate small and large eddies instead of time-averaging as in RANS. The computational requirements to perform a LES simulation are much higher than that of a RANS simulation, but the accuracy is also generally much higher. Direct numerical simulation (DNS) is the most computationally expensive but also the most accurate. DNS solves the Navier-Stokes equations directly without the need for a turbulence model. Kim et al. [32] showed that DNS of a channel flow with a Reynolds number based on channel height of 6000 required 2-4 million mesh points. The number of mesh points required increases as the Reynolds number increases, due to the increased range of eddy scales as the Reynolds number is increased. An estimate correlating the number of points needed based on the channel height Reynolds number is given in equation (2-3) [33].

$$
N=\left(0.088 * R e_{h}\right)^{9 / 4}
$$

It can be seen from this relationship that even at modest Reynolds numbers DNS requirements are steep and can exceed the limitations of modern computing technology.

\subsection{RANS AND DESCRIPTION OF Closure Problem}

For a RANS simulation, the conservation of mass, momentum, and energy partial differential equations are time-averaged as opposed to directly solving as is the case with DNS. The time averaging process leaves behind a cross multiplying term involving the fluctuating velocity components. The units on this term $\left(\rho \overline{u_{\imath} u_{\jmath}}\right)$ has units of stress and is commonly known as a Reynolds Stress. There are nine terms in the Reynolds stress tensor but due to its symmetric nature only six are unique. In addition to the Reynolds stresses, turbulent fluxes of the form $\left(\rho \overline{u_{J} \varphi^{\prime}}\right)$ are also introduced. Equations (2-4) through 
(2-7) give the RANS equations as well as the scalar transport equation. Temperature is the scalar shown in the scalar transport equation with the source term included.

$$
\begin{gathered}
\frac{\partial \bar{U}_{i}}{\partial x_{i}}=0 \\
\frac{\partial \bar{U}_{i}}{\partial t}+\bar{U}_{j} \frac{\partial \bar{U}_{i}}{\partial x_{j}}=\frac{1}{\rho} \frac{\partial}{\partial x_{j}}\left(-\bar{P} \delta_{i j}+S_{i j}-\rho \overline{u_{\imath} u_{\jmath}}\right) \\
S_{i j}=\mu\left(\frac{\partial \bar{U}_{i}}{\partial x_{j}}+\frac{\partial \bar{U}_{j}}{\partial x_{i}}\right) \\
\frac{\partial \Phi}{\partial t}+\bar{U}_{j} \frac{\partial \Phi}{\partial x_{j}}=\frac{\partial}{\partial x_{j}}\left(\Gamma \frac{\partial \Phi}{\partial x_{j}}-\rho \overline{u_{j} \varphi^{\prime}}\right)+S_{\Phi}
\end{gathered}
$$

By decomposing the instantaneous quantities in the Navier-Stokes equations into the mean and fluctuating components additional unknowns were introduced. No additional equations were gained and the system of equations is not closed. This is, in essence, the closure problem that necessitates the need for turbulence models.

\subsubsection{SELECTION OF TURBULENCE MODEL}

Turbulent closure models are needed to account for the Reynolds stress terms. No single turbulence closure model spanning across all applications exists for the RANS

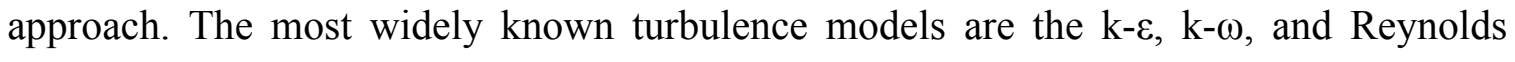
Stress Model (RSM). The most common RANS turbulence models can be classified based on the number of extra transport equations introduced to be solved in conjunction with the RANS flow equations. The k- $\varepsilon$ and $k-\omega$ models have two additional transport equations while seven additional transport equations are needed for the Reynolds Stress Model (RSM) [34]. It is necessary to use past research to compare experimental results to computational results and select the turbulence model most accurate to the studied 
phenomenon. Harrison and Bogard [35] studied the prediction of numerous turbulence models on straight film cooling holes and determined that the standard $k-\omega$ model resulted in the closest agreement with laterally averaged adiabatic effectiveness from experiments, but deviated from experiments when the centerline adiabatic effectiveness was considered. In order to keep consistent with past research $[12,13,24]$ the $\mathrm{k}-\omega$ model was selected with SST modification by Mentor [36].

In the two-equation models the Reynolds stresses are computed with the Boussinesq approximation to relate the Reynolds stress tensor to the mean velocity gradients as shown in equation (2-8). The turbulence kinetic energy per unit mass $(k)$ is defined in equation (2-9).

$$
\begin{gathered}
-\rho \overline{u_{\imath} u_{\jmath}}=2 \mu_{t} S_{i j}-\frac{2}{3} \rho k \delta_{i j} \\
k=\frac{1}{2} \overline{u_{\imath} u_{\imath}}
\end{gathered}
$$

For the k- $\omega$ model the eddy or turbulent viscosity is given by equation (2-10) and the effective turbulent thermal conductivity is given by equation (2-11).

$$
\begin{gathered}
\mu_{t}=\rho k / \omega \\
k_{t}=k+\mu_{t} c_{p} / P r_{t}
\end{gathered}
$$

The additional transport equations introduced in the standard $k-\omega$ model are given in equations (2-12) through (2-15) and the description of each term in the equations is shown in Figure 15. The constants in the equation $\left(\sigma_{\mathrm{k}}, \sigma_{\omega}, \gamma_{1}, \beta_{1}, \beta^{*}\right)$ can be found in [34] but are known to be changed in different implementations of the model. 


$$
\begin{gathered}
\frac{\partial \rho k}{\partial t}+\bar{U}_{j} \frac{\partial \rho k}{\partial x_{j}}=\frac{\partial}{\partial x_{j}}\left(\left(\mu+\frac{\mu_{t}}{\sigma_{k}}\right) \frac{\partial k}{\partial x_{j}}\right)+P_{k}-\beta^{*} \rho k \omega \\
P_{k}=\left(2 \mu_{t} S_{i j} \cdot S_{i j}-\frac{2}{3} \rho k \frac{\partial \bar{U}_{i}}{\partial x_{j}} \delta_{i j}\right) \\
\frac{\partial \rho \omega}{\partial t}+\bar{U}_{j} \frac{\partial \rho \omega}{\partial x_{j}}=\frac{\partial}{\partial x_{j}}\left(\left(\mu+\frac{\mu_{t}}{\sigma_{\omega}}\right) \frac{\partial \omega}{\partial x_{j}}\right)+P_{\omega}-\beta_{1} \rho \omega^{2} \\
P_{\omega}=\gamma_{1}\left(2 \rho S_{i j} \cdot S_{i j}-\frac{2}{3} \rho \omega \frac{\partial \bar{U}_{i}}{\partial x_{j}} \delta_{i j}\right)
\end{gathered}
$$

\begin{tabular}{|c|c|c|c|c|c|}
\hline $\begin{array}{l}\text { Rate of } \\
\text { change } \\
\text { of } k \text { or } \omega\end{array}$ & $+\begin{array}{c}\text { Transport of } k \\
\text { or } \omega \text { by } \\
\text { convection }\end{array}$ & $\begin{array}{c}\text { Transport of } k \text { or } \\
\omega \text { by turbulent } \\
\text { diffusion }\end{array}$ & $\uparrow$ & $\begin{array}{l}\text { Rate of } \\
\text { production of } \\
k \text { or } \omega\end{array}$ & $\begin{array}{c}\text { Rate of } \\
\text { dissipation of } \\
\text { k or } \omega\end{array}$ \\
\hline
\end{tabular}

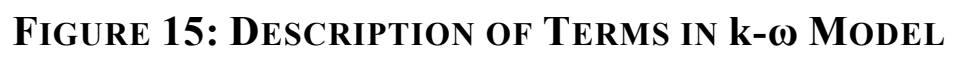

Mentor [36] modified the original k- $\omega$ model with the inclusion of an additional source term on the right hand side of the equation. The modified $\omega$-equation is shown in equations (2-16) through (2-18) and the constants can also be found in [34]. The constants for the simulations modeled were left as the defaults in Star-CCM+ and can be found in the user's guide [37].

$$
\begin{gathered}
\frac{\partial \rho \omega}{\partial t}+\bar{U}_{j} \frac{\partial \rho \omega}{\partial x_{j}}=\frac{\partial}{\partial x_{j}}\left(\left(\mu+\frac{\mu_{t}}{\sigma_{\omega, 1}}\right) \frac{\partial \omega}{\partial x_{j}}\right)+P_{\omega}-\beta_{2} \rho \omega^{2} \\
P_{\omega, 1}=\gamma_{2}\left(2 \rho S_{i j} \cdot S_{i j}-\frac{2}{3} \rho \omega \frac{\partial \bar{U}_{i}}{\partial x_{j}} \delta_{i j}\right) \\
P_{\omega, 2}=2 \frac{\rho}{\sigma_{\omega, 2} \omega} \frac{\partial k}{\partial x_{i}} \frac{\partial \omega}{\partial x_{i}}
\end{gathered}
$$




\subsection{Control Volume and Computational Setup}

A control volume was selected consistent with previous studies in the open literature, to allow for comparison of the performance of a single film cooling geometry. A control volume is selected to encompass the region of influence of a single film cooling hole. This control volume is shown in the blue transparent region of Figure 16. The surrounding apparatus is the full test section of the experimental facility.

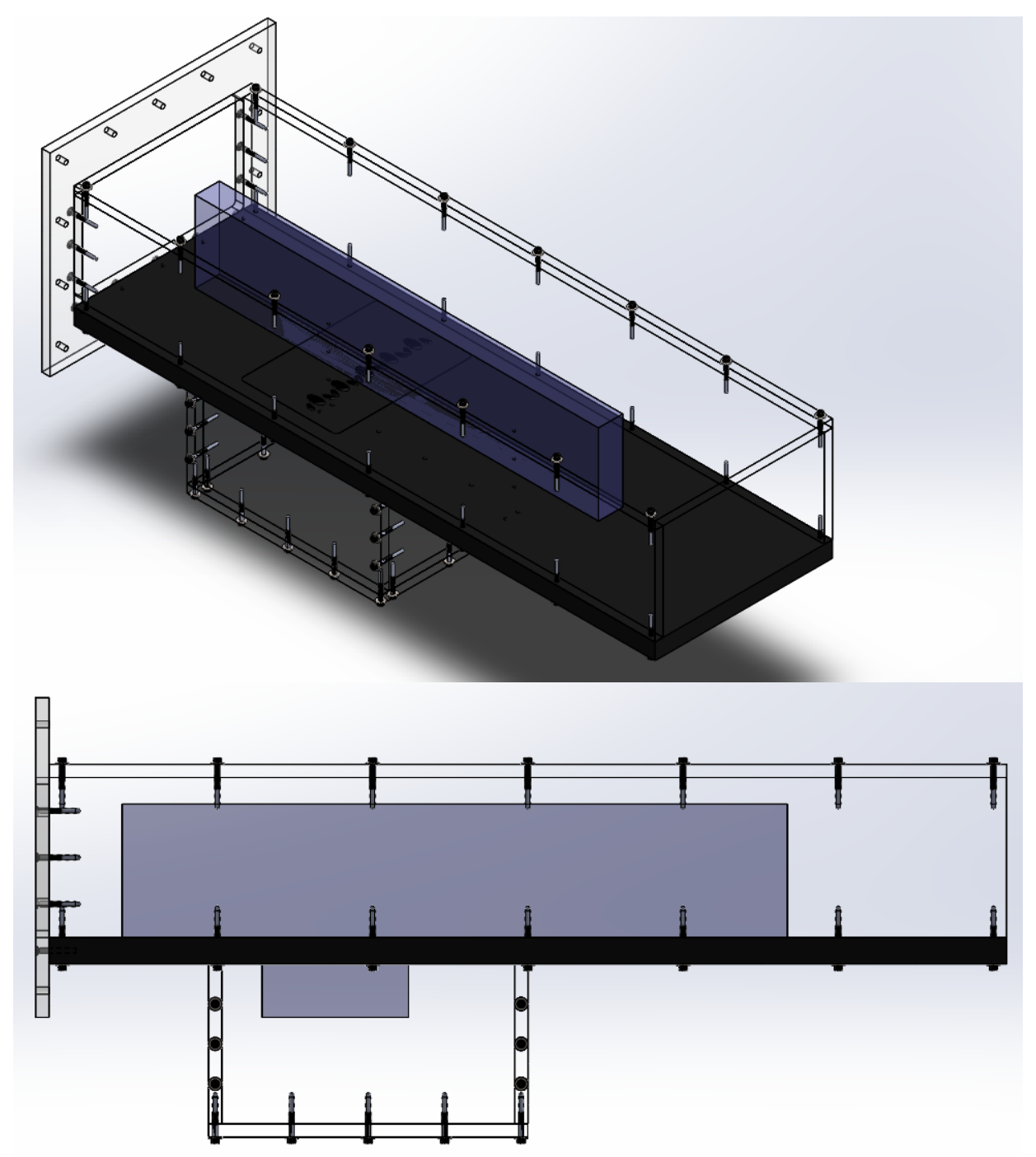

Figure 16: Isometric And Side View of CFD Control Volume Compared TO WIND TUNNEL 
The control volume in the present work is the same as in Heidmann $[12,13]$ and Hunley et al. [24] for ease of direct comparison of results. All dimensions for the control volume were normalized by the main hole diameter of the AVH. A total pressure inlet boundary condition was imposed 19 hole diameters upstream of the cooling holes and a pressure outlet was imposed 30 hole diameters downstream. The top plane extends 10 hole diameters above the test surface and has been assumed to be sufficiently high enough to be considered a symmetry plane. The width of the full control volume of the AVH is 3 hole diameters from the symmetry plane to opposite symmetry plane as shown in Figure 17. It is important to note that the computational domain for the nine adiabatic and nine heat transfer cases was modeled as half of the full domain as shown in Figure 17, to save computational time. A symmetry plane was introduced at the main hole centerline, and the domain extends 1.5 hole diameters in width to the outer symmetry plane. This is valid for the steady RANS solver since no flow is crossing through the symmetry planes. All unsteady work includes the full domain to account for unsteady flow crossing the plane along the main hole centerline. The plenum was set to a total pressure inlet and was set to a few percent higher than the inlet static pressure through a trial and error process to achieve the blowing ratio desired. 


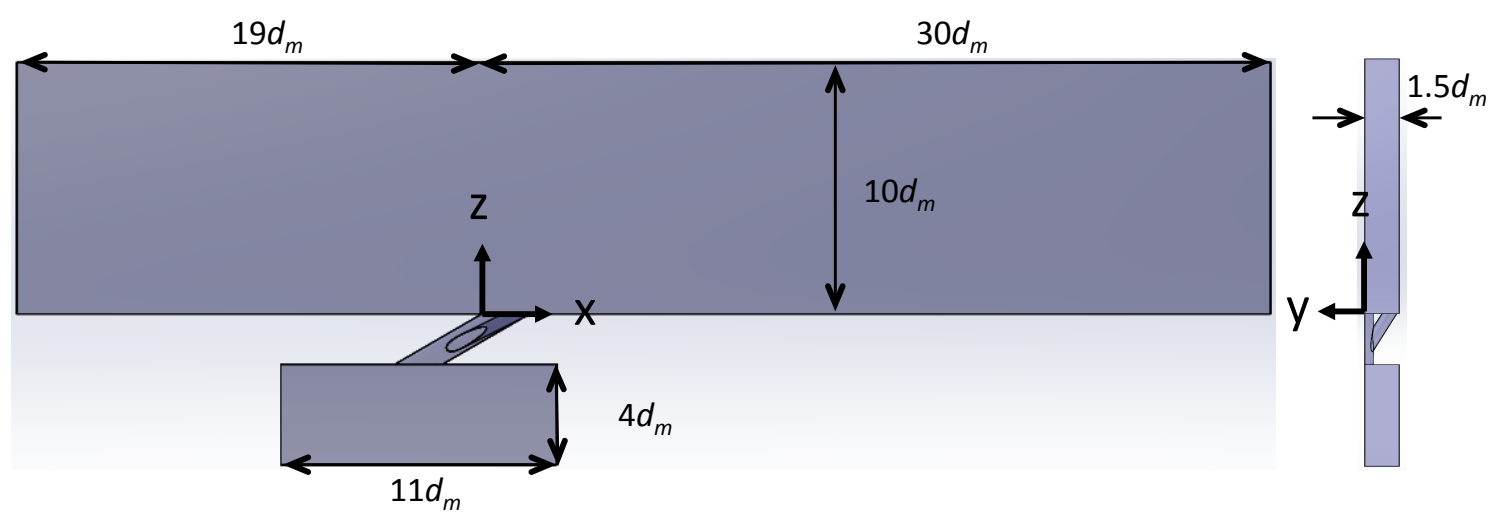

\section{Figure 17: Front, Side, And Top View of Control Volume Modeled For STEADY RANS}

Air was used as the fluid in both the freestream and the plenum. The viscosity of the air was found through a 0.7 power law [38]. The ideal gas law was used to predict the density of the fluid, and the specific heat of the air was held constant. The thermal conductivity of the air was found using Sutherland's law with a reference value of $0.02414 \mathrm{~W} / \mathrm{m}-\mathrm{K}[39]$.

\subsubsection{ORIGINAL RANS CASES AND HEAT TRANSFER CASES}

A multi-block structured hexahedral grid was produced using GridPro ${ }^{\mathrm{TM}}$ for the nine adiabatic cases and the nine heat transfer cases. This grid contained 2.5 million hexahedral cells and employed viscous clustering near to the solid walls with a $y+$ value less than 1.0 at all locations. Nominally the $y+$ value was on the order of 0.1 at nearly all locations along the top surface. A stretching ratio of 1.2 was used normal to the viscous walls in conjunction with standard CFD practice for film cooling studies. Figure 18 shows the structured multi-block grid created in GridPro ${ }^{\mathrm{TM}}$, with each block indicated by a different color. This grid was built using the same setup as Heidmann [12] and is the "fine" grid from the study. A coarse grid of approximately 300,000 cells was used to 
check the sensitivity to the grid. It was found that the differences between the coarse and fine grid results were less than 10 percent indicating that the grid is approaching grid independence.

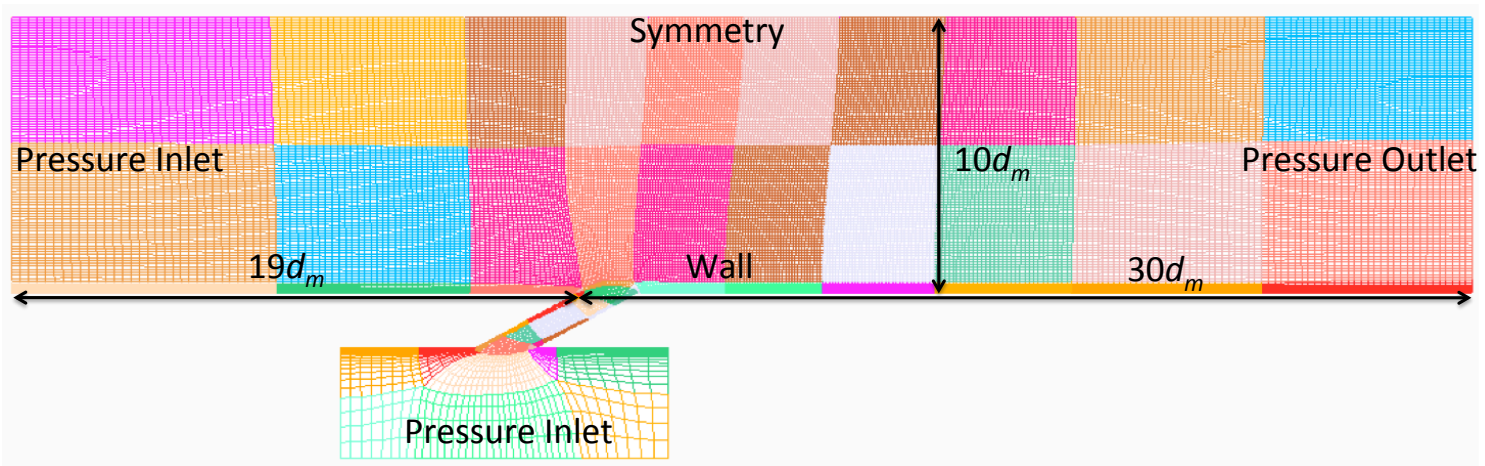

\section{Figure 18: Computational Grid for Adiabatic and Heat Transfer Cases}

The ratio of the static pressure of the outlet to the total pressure of the inlet was set to the isentropic value of $\mathrm{p} / \mathrm{p}_{0} 0.9725$ to produce a nominal Mach number of 0.2 in the freestream flow. The plenum total pressure was set approximately 8 percent higher than the inlet total pressure to produce a blowing ratio of 2.0. The plenum total temperature was set to 0.5 times the freestream total temperature to provide a temperature difference considered representative of engine conditions [12]. A turbulence intensity of 1.0 percent and a length scale normalized by main cooling hole diameter of 1.0 was specified for the plenum. A Reynolds number based on the main film cooling hole diameter of 11,300 was matched to previous work $[12,13,24]$.

A heat flux of zero was supplied at all walls for the adiabatic cases to provided the film cooling effectiveness needed for calculation of the heat transfer coefficient. For the heat transfer cases a specified wall heat flux was applied at the test surface to allow for the calculation of the heat transfer coefficient needed to compute the NHFR. The 
selection of the heat flux is not important in the calculation of the heat transfer coefficient, only that there is a non-zero heat flux at the wall. The heat transfer coefficient is independent of the temperature and only depends on the fluid properties and flow conditions.

Turbulence was prescribed for the $k$ - $\omega$ turbulence model as specified turbulence intensity and length scale values at the inlet and outlet planes based on the cases being studied as described in section 1.4. Turbulence intensities and length scales were varied with intensities of 5, 10, and 20 percent as well as length scales of 1, 3, and 6 main hole diameters, resulting in nine adiabatic and nine heat transfer cases. A summary of these nine cases is given in Table 3 . The numerical simulations for steady cases were carried out on a six-core intel based i7 desktop computer with 32 GB of DDR3 RAM. The adiabatic cases required approximately 500-1000 CPU hours to converge or roughly a week of actual time. The heat transfer cases required some additional computational time, on the order of $25-50 \%$ more time depending on the length scale and turbulence intensity. Convergence was achieved for each case when all of the residuals were reduced by three orders of magnitude and there was no observable change in the surface temperature prediction downstream of the holes for 1000 iterations. This was accomplished using area-averaged monitors of the surface temperature as well as discrete monitors downstream of the cooling holes at the surface as well as three hole diameters off of the surface. 
Table 3: Turbulence Cases

\begin{tabular}{|c|c|c|}
\hline Case Number & Turbulence Intensity $(\mathrm{Tu})$ & Length Scale $\left(\Lambda_{\mathrm{x}} / \mathrm{d}_{\mathrm{m}}\right)$ \\
\hline 1 & 5 & 1 \\
\hline 2 & 10 & 1 \\
\hline 3 & 20 & 1 \\
\hline 4 & 5 & 3 \\
\hline 5 & 10 & 3 \\
\hline 6 & 20 & 3 \\
\hline 7 & 5 & 6 \\
\hline 8 & 10 & 6 \\
\hline 9 & 20 & 6 \\
\hline
\end{tabular}

\subsubsection{FLAT PLATE STUDY}

A shortened CFD study was conducted in Star-CCM+ to determine the heat transfer coefficient with no film cooling. The purpose behind this study was to compare the prediction of heat transfer found from the simulations to a Nusselt number correlation from Incropera et al. [40] and a Stanton number correlation from Kays and Crawford [17] for a flat plate in a turbulent, parallel flow with constant heat flux at the wall. These correlations are given in equations (2-19) and (2-20) respectively.

$$
\begin{aligned}
& N u_{x}=0.0296 \operatorname{Re}_{x}^{4 / 5} \operatorname{Pr}^{1 / 3} \quad 0.6 \lesssim \operatorname{Pr} \lesssim 60 \\
& S t P r \\
& 0.4=.030 \operatorname{Re}_{x}^{-0.2}
\end{aligned}
$$

The mesh for the flat plate study was resolved to be nearly grid independent with the temperature predictions changing by less than $1 \%$ when the grid resolution was doubled. The computational time for this study was minimal and multiple cases could be processed at once, overnight. The results show that prediction in Star-CCM+ using the same turbulence model as implemented in the rest of the study predicts a higher heat transfer coefficient that either of the correlations. This is shown graphically in Figure 19. 


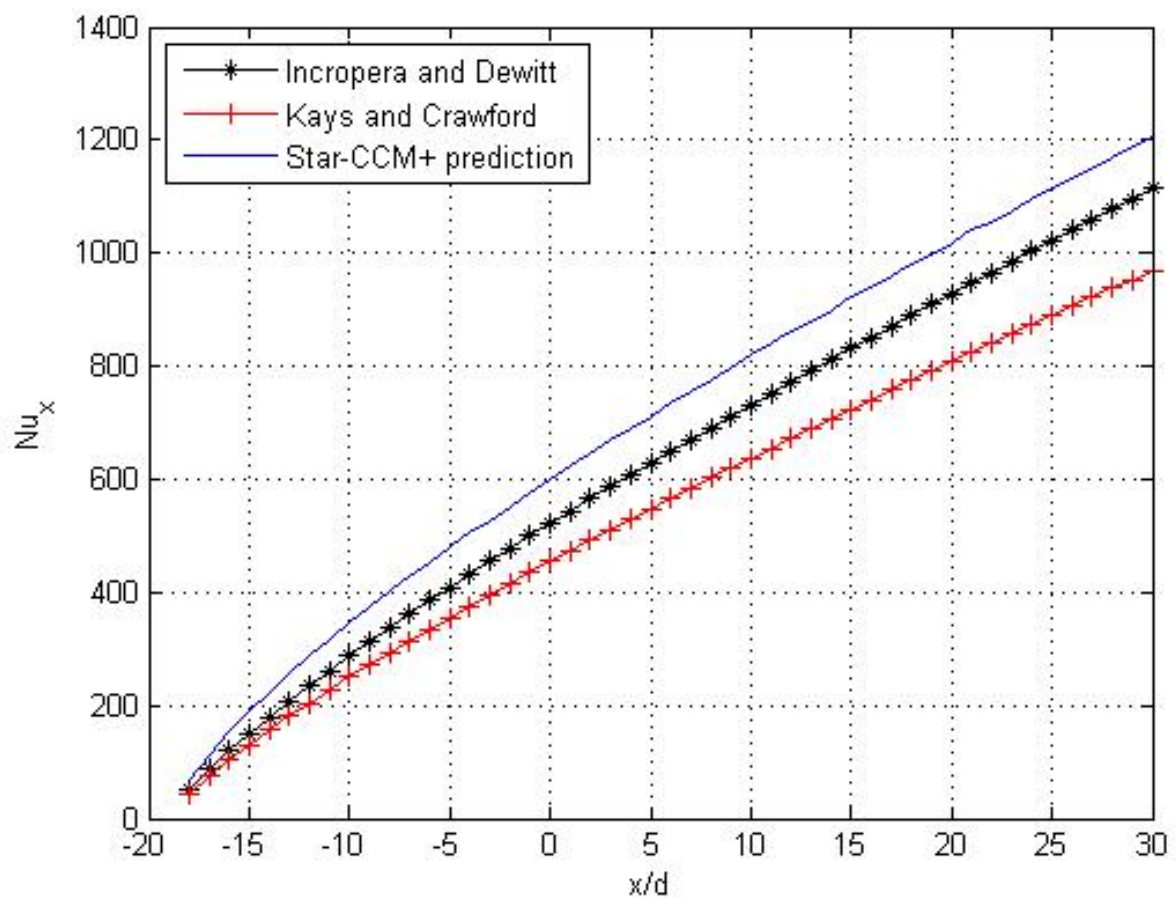

Figure 19: Flat Plate Nusselt Number Comparison

In the author's opinion, it is best to use the prediction of the heat transfer coefficient from the flat plate simulation to compare to the film cooling simulations as opposed to the turbulent heat transfer correlations. Hence, the Star-CCM+ predicted heat transfer coefficient has been used in the heat transfer analysis of chapter 3 for the heat transfer coefficient with no film cooling $\left(\mathrm{h}_{0}\right)$.

\subsubsection{UNSTEADY RANS CASES}

For the unsteady analysis of the AVH performance, the full domain of the control volume was required. In an effort to save time setting up a structured hexahedral grid in GridPro $^{\mathrm{TM}}$, a trimmed hexahedral grid was created in Star-CCM+. A trimmed hexahedral mesh is predominantly a hexahedral mesh with minimal cell skewness. The bulk of the mesh is created as a hexahedral mesh and then trimmed using the input surfaces. This produces a hexahedral mesh that is nearly inline with the freestream flow direction and 
can produce highly accurate results in these areas. Near to the surface the cells that are trimmed are polyhedral cells. Viscous clustering was employed at the near wall locations with a $y+$ near 1.0. An additional level of refinement was done to increase the resolution near to the coolant jet interaction with the mainstream. This was done by enclosing a box shaped volume around the coolant jet starting as shown by the increased mesh density shown in Figure 20. The resulting enclosed cells were 60 percent of the base mesh size in this area.

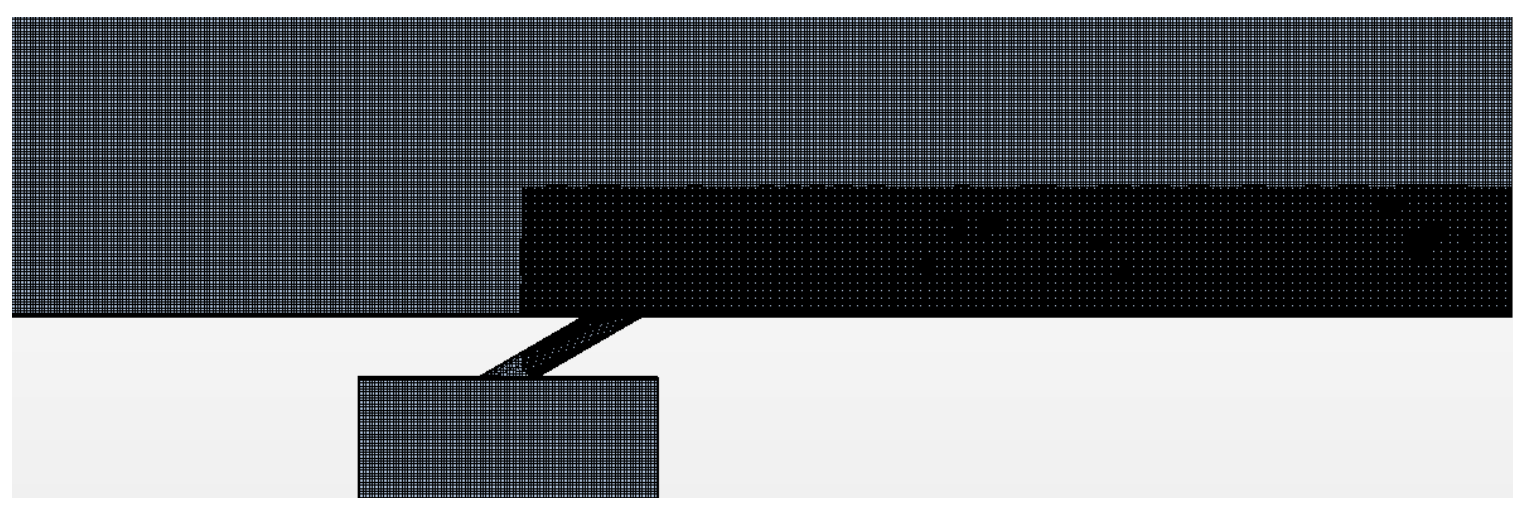

Figure 20: URANS Mesh With Volumetric Controls

The trimmed mesh used for the URANS cases contains 4.5 million cells. The same boundary conditions were implemented in the URANS simulations as in the case of the steady RANS. The main difference is the inclusion of the unsteady time step. In essence, URANS captures fluctuations or unsteady behavior in the mean quantities of the flow. The time step used for these simulations was chosen based on the grid refinement to be 1E-6 seconds to capture the fluctuations in the mean flow quantities. This allow for a Courant-Friedrichs-Lewy (CFL) condition to be less than 1.0, although an implicit solver was utilized negating any stability requirements. 
Pressure and temperature conditions at the inlet, outlet, and plenum were prescribed to be the same as in the adiabatic cases. Turbulence conditions in the plenum were also consistent with the adiabatic cases. Due to limited impact of length scale as reported in Repko et al. [14] and limited computational power, the unsteady test cases were studied at turbulence intensities of 5,10 and $20 \%$. The turbulence length scale $\left(\Lambda_{x} / d_{m}=1\right)$ in the three unsteady cases was selected to match as closely as possible to the length scale in the experimental facility.

\subsubsection{ADDITIONAL CASES TO MATCH FUTURE EXPERIMENTAL WORK AT WVU}

A computational setup within Star-CCM+ has been created as a tool for future students to complete additional RANS simulations on the AVH as tested in this study. Simple modifications to the boundary conditions can allow for testing of any number of parameters including but not limited to; the blowing ratio, density ratio, Reynolds number, and approaching boundary later thickness. This will be a valuable tool for comparisons to future experimental data obtained. The incoming boundary layer profile can be input into the model as a table using the results of hot-wire traverses from the wind tunnel. This will provide more accurate RANS CFD results than allowing the boundary layer to develop from the start of the control volume. 


\subsection{EXPERIMENTAL SETUP}

Figure 21 shows the full CAD assembly of the experimental wind tunnel, shown in scale proportionally, but without dimensions. The blower is connected to a diffuser through a section of flexible duct to dampen any oscillations due to the rotation of the blower. The flow is diffused to lower the velocity entering the flow straightener section. The flow is straightened by way of a honeycomb mesh to eliminate as much swirl as possible before entering the test section. From the flow straightener the flow passes through a nozzle to reaccelerate before passing through two mesh screens that will be used to induce a step temperature change in the flow. After passing through the mesh screens the flow encounters a turbulence grid. Three turbulence conditions currently can be modeled in the tunnel. With no grid the turbulence intensity is $1 \%$ and has a characteristic length scale of $\left(\Lambda_{\mathrm{x}} / \mathrm{d}_{\mathrm{m}}\right)$ of 6.4 . The two turbulence grids used were made from $1 / 4 "$ and $1 / 2 "$ aluminum bars. The turbulence decays as one moves away from the bars to $7.5 \%$ and $11.7 \%$ at the leading edge of the holes, for the $1 / 4$ " and $1 / 2$ " bar respectively. The length scale based on the main film cooling hole diameter is approximately 1.0 for each of the turbulence grids. An actively blown turbulence grid may be used in the future to generate higher turbulence intensities and length scales.

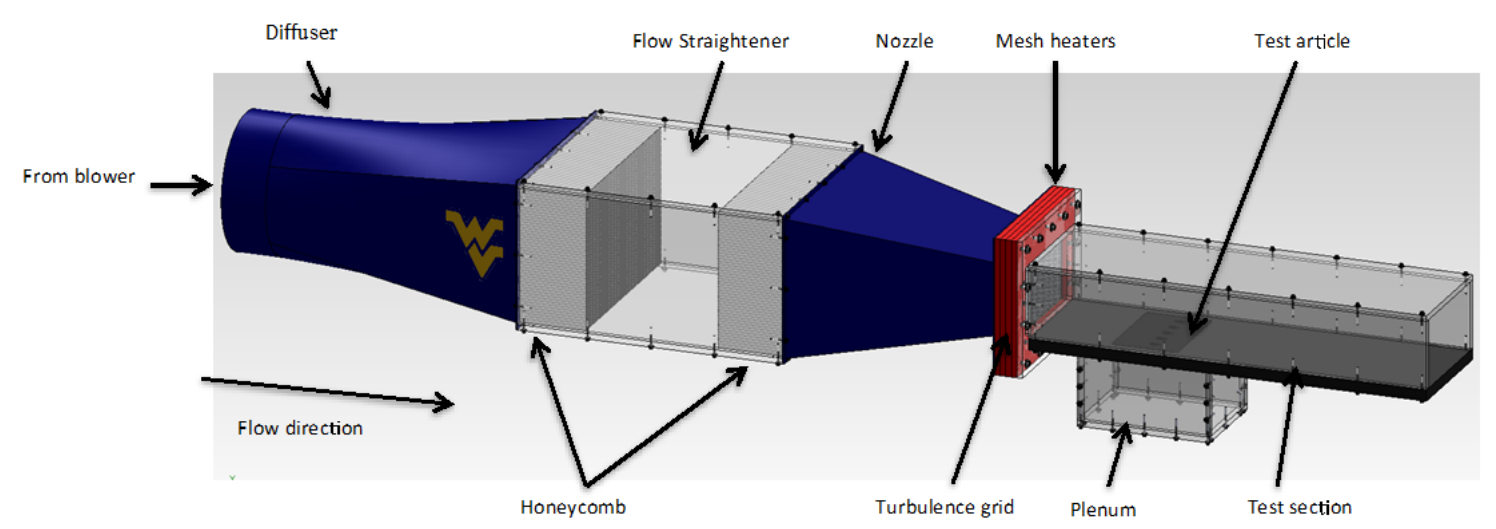

Figure 21: CAD DRAWING OF EXPERIMENTAL FACILITY 
The properties of the freestream are measured using thermocouples, a pitot static probe and a constant temperature hotwire anemometer. The coolant air supplying the plenum is routed from a compressor. The coolant can either be at room temperature or cooled using a passive liquid nitrogen heat exchanger. The surface temperature of the test plate is measured using an infrared thermography technique with a FLIR A655sc camera with a $45^{\circ}$ lens. The test surface can be viewed through a stretched polyurethane sheet or an Edmund Optics infrared window. Calibration of the IR camera is unique to the material that the surface is viewed through and is covered in [41].

The test article is modeled as a semi-infinite solid undergoing a transient heat transfer process. The heat equation can be simplified to its one-dimensional form as shown in equation (2-21). The initial temperature condition for a typical transient conduction problem is given in equation (2-22). It is assumed that convective heat transfer at the wall is equal to the conduction at the wall. The reference temperature driving the convection process is the film temperature $\left(\mathrm{T}_{\mathrm{f}}\right)$. The film temperature is an approximation of the temperature inside the convective boundary layer and is a function of the local mixing of the coolant stream with the main stream as defined in equation (2-23).

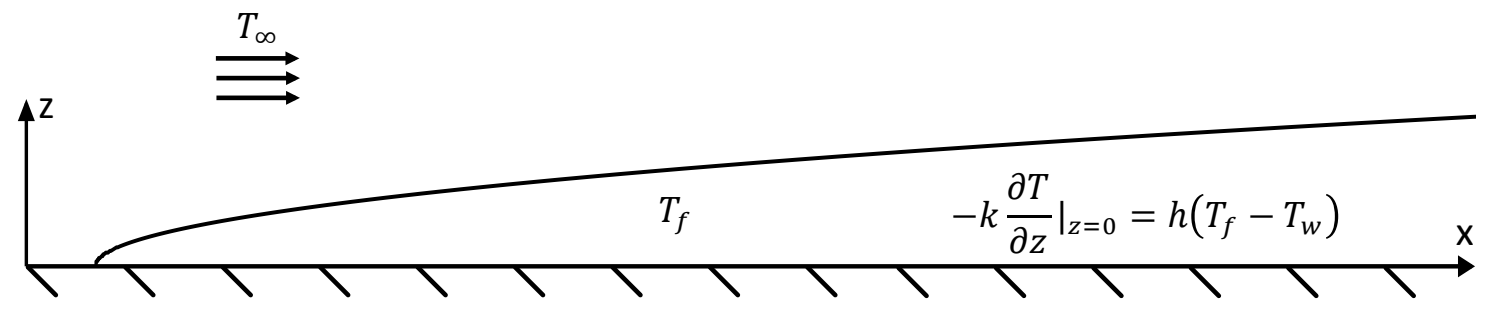

Figure 22: Transient Heat Transfer With Convection and Semi-Infinite SOLID ASSUMPTION

$$
\frac{\partial^{2} T}{\partial z^{2}}=\frac{1}{\alpha} \frac{\partial T}{\partial t}
$$




$$
\begin{gathered}
T(z, 0)=T_{i} \& \mathrm{~T}(z, \infty)=T_{i} \\
T_{f}=\frac{T_{\infty}+T_{w}}{2} \\
-\left.k \frac{\partial T}{\partial z}\right|_{z=0}=\left.h\left(T_{f}-T_{w}\right)\right|_{t>0}
\end{gathered}
$$

From the above initial conditions and boundary conditions solutions of the form in equation (2-25) can be obtained. Through a simple modification for film cooling situations the equation becomes equations (2-26)

$$
\begin{gathered}
\frac{T_{w}-T_{i}}{T_{\infty}-T_{i}}=1-\exp \left(\frac{h^{2} \alpha t}{k^{2}}\right) \operatorname{erfc}\left(\frac{h \sqrt{\alpha t}}{k}\right)=1-\operatorname{erfcx}\left(\frac{h \sqrt{\alpha t}}{k}\right) \\
\frac{T_{w}-T_{i}}{T_{f}-T_{i}}=1-\operatorname{erfcx}\left(\frac{h \sqrt{\alpha t}}{k}\right)
\end{gathered}
$$

where $\operatorname{erfc}$ is the complementary error function and erfcx is the scaled complementary error function given in equations (2-27) and (2-28) respectively.

$$
\begin{gathered}
\operatorname{erfc}(x)=1-\operatorname{erf}(x) \\
\operatorname{erfcx}(x)=\exp \left(x^{2}\right) * \operatorname{erf}(x)
\end{gathered}
$$

Ekkad [42] proposed using IR thermography to measure the surface temperature at two instants in time $\left(\mathrm{T}_{\mathrm{w} 1}\right)$ at time $\left(\mathrm{t}_{1}\right)$ and $\left(\mathrm{T}_{\mathrm{w} 2}\right)$ at time $\left(\mathrm{t}_{2}\right)$ at every point to determine the heat transfer coefficient and film temperature in a single test, by solving equations (2-29) and (2-30) simultaneously.

$$
\frac{T_{w 1}-T_{i}}{T_{f}-T_{i}}=1-\operatorname{erfcx}\left(\frac{h \sqrt{\alpha t_{1}}}{k}\right)
$$




$$
\frac{T_{w 2}-T_{i}}{T_{f}-T_{i}}=1-\operatorname{erfcx}\left(\frac{h \sqrt{\alpha t_{2}}}{k}\right)
$$

These equations have been solved in MATLAB simultaneously through the code given in Appendix A. Through the use of the parallel computing toolbox the code was able to solve for the unknown heat transfer coefficient and film temperature in less than 30 seconds. Serial computation of the code required greater than 20 minutes.

The heat transfer results of this wind tunnel study will be compared to the results of the present study once the tunnel is fully functional and the AVH test plate is installed. Experiments and numerical simulations are complementary in their validation of each other's results. The future results of this wind tunnel will be valuable when considering the numerical results present herein. 
ANALYSIS AND RESUlts

\subsection{Summary OF Adiabatic CASES FOR AVH}

The nine adiabatic cases were all solved using a steady RANS solver in the commercially available software, Star-CCM+. The main quantity of interest from the solved RANS equations is the temperature at the wall, with the overall desired results is to achieve a maximum wall cooling over as large an area as possible, for the given blowing ratio and density ratio. The means of determining the initial effect of turbulence on the AVH performance is through a comparison of these adiabatic wall temperatures, nondimensionalized, and given as the adiabatic film cooling effectiveness from equation (1-16). The effectiveness is analyzed as a contour on the tested surface, along the main hole centerline, span-averaged across the computational domain, and as an area-averaged quantity downstream of the holes. The contour plots give a general picture which is used to allow the centerline, span-averaged, and area-averaged values to be put into a perspective so that one might be able to qualitatively understand the physics of the flow for a given set of conditions and geometry. Analyzing the effectiveness along the main hole centerline can provide insight into the amount of jet lift off that is present for the main cooling hole. The results of averaging across the span of the domain gives a detailed 
depiction of the performance of a given film cooling geometry at a set of conditions, and is the best quantitative look into how well the geometry functions. Area-averaging the effectiveness downstream of the hole paints the broadest picture of the film cooling functionality. A limitation of the area-averaged effectiveness that is to be noted is hot streaks and hot spots may not have a large effect on the reported value of the areaaveraged effectiveness but could be sufficiently large enough to cause localized thermal failure of components.

Table 3 is duplicated below to show again a summary of the parameters describing the nine RANS adiabatic cases.

Table 3: Turbulence Cases

\begin{tabular}{|c|c|c|}
\hline \hline Case Number & Turbulence Intensity $(\mathrm{Tu})$ & Length Scale $\left(\Lambda_{\mathrm{x}} / \mathrm{d}_{\mathrm{m}}\right)$ \\
\hline 1 & 5 & 1 \\
\hline 2 & 10 & 1 \\
\hline 3 & 20 & 1 \\
\hline 4 & 5 & 3 \\
\hline 5 & 10 & 3 \\
\hline 6 & 20 & 3 \\
\hline 7 & 5 & 6 \\
\hline 8 & 10 & 6 \\
\hline 9 & 20 & 6 \\
\hline
\end{tabular}

\subsubsection{Contour Plots of EFFECTIVENESS}

Figure 23 is shown to illustrate the typical coverage of a straight film cooling flow at high blowing ratio. It can be seen that there is a large amount of area downstream of a hole that is not covered at all by the coolant. An AVH geometry has a much better coolant coverage than the straight film cooling hole case at high blowing ratio. 


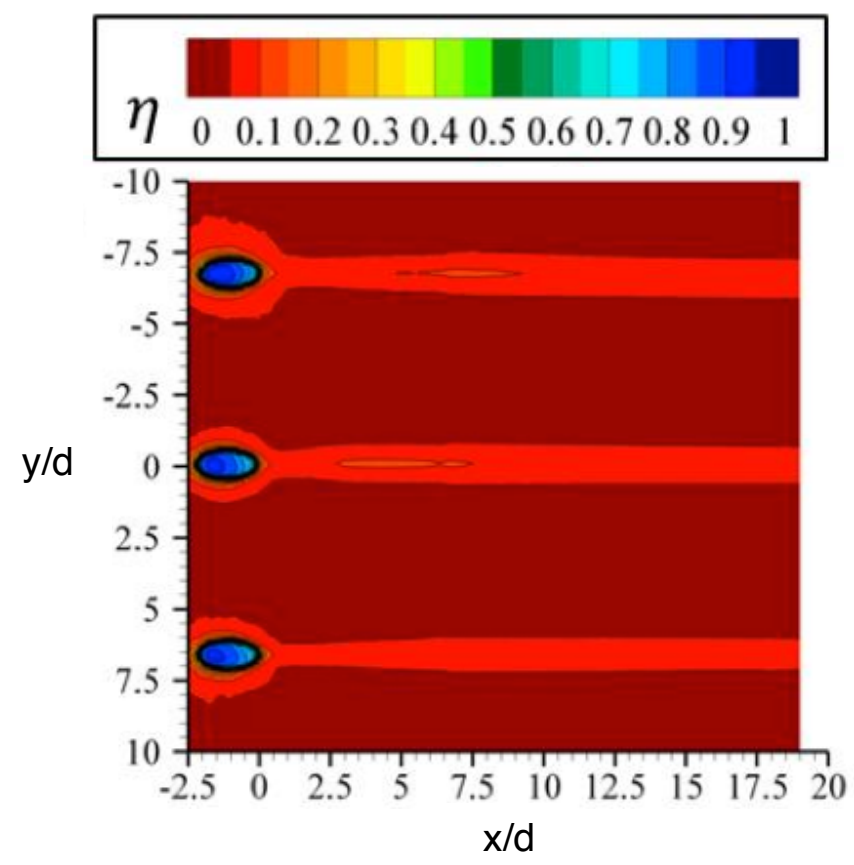

\section{Figure 23: Typical Film Cooling Coverage of Straight Film Cooling Hole at High Blowing Ratio (BR = 2) [43]}

Figure 24 shows contour plots of the computed adiabatic effectiveness for the test surface, for all nine turbulence cases, mirrored across the main hole centerline for clarity. The hole spacing is three hole diameters as is consistent with practice in real engines for cylindrical holes and is shown in the first contour plot in the figure. For the low turbulence cases, the lateral spreading of the coolant down stream of the hole is less prominent than in the higher turbulence cases. This is highlighted by a black oval shown in the top contour plot with the lowest turbulence intensity and smallest length scale. As the turbulence intensity increases the lateral spreading of the coolant occurs much further upstream and provides better coverage and thermal protection than at lower turbulence intensities. A region of interest in between the main and side holes highlighted in the figure shows a "hot streak." This hot streak is shortened with increasing turbulence intensity, as can be shown by comparing the black oval shown in the bottom contour plot to the longer oval in the top contour plot. Trends showing improvement to the cooling 
effectiveness with increasing turbulence intensity match the trends for cylindrical holes at high blowing ratio found in Bons et al. [29] and the trends found for shaped holes found in Saumweber et al. [30, 31].

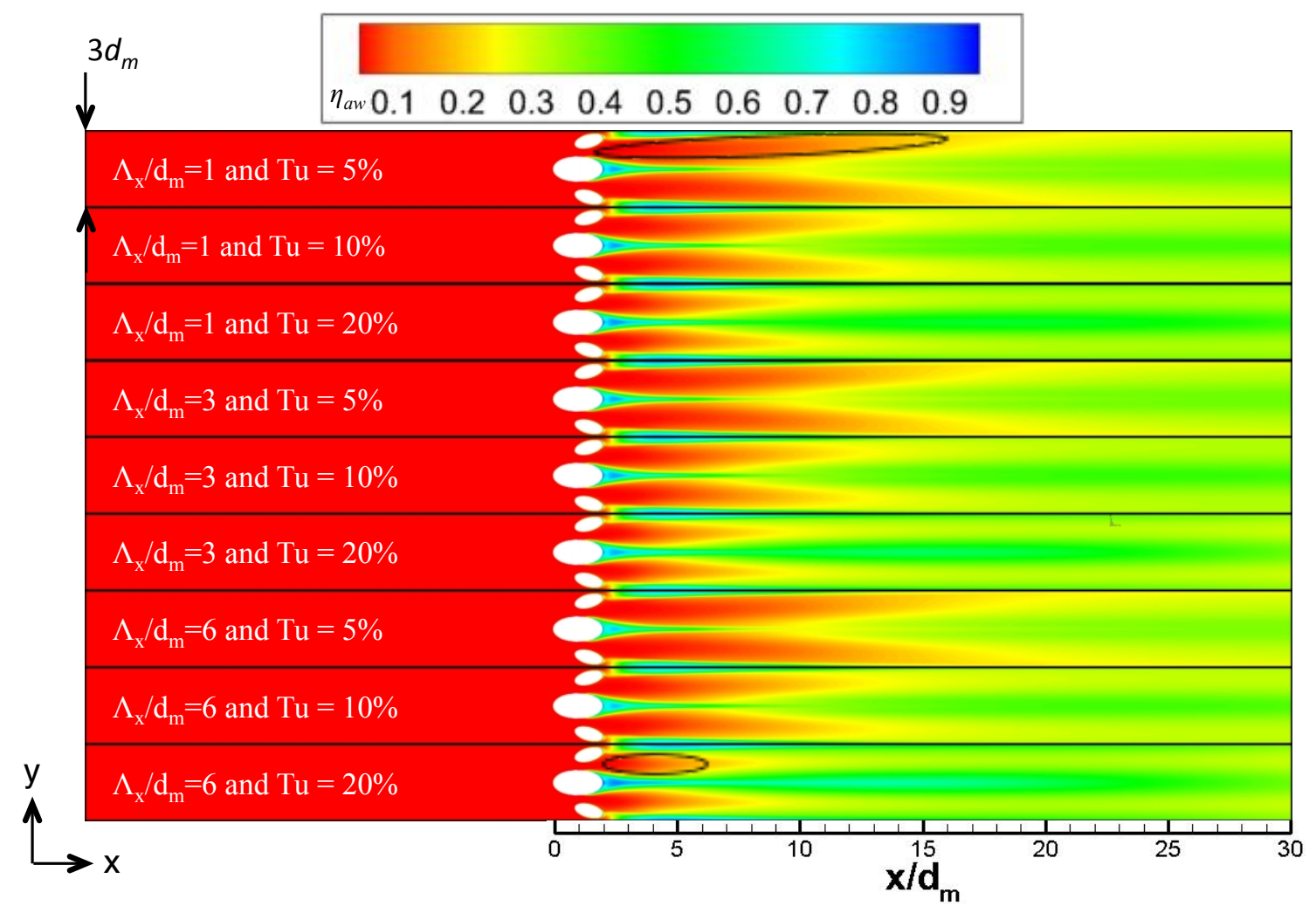

\section{Figure 24: Contour Plots of Adiabatic Effectiveness for Nine Turbulence Conditions}

It is not trivial to identify any effect of length scale $\left(\Lambda_{\mathrm{x}} / \mathrm{d}_{\mathrm{m}}\right)$ on the film cooling effectiveness by looking at contour plots alone. The effect of length scale is more easily discerned with the centerline and span-averaged effectiveness, as will be discussed in the following sections. 


\subsubsection{CENTERLINE EFFECTIVENESS}

At any streamwise point downstream of a cooling hole, the maximum cooling effectiveness is generally along the hole centerline. This maximum can be located by comparing centerline effectiveness plots. Centerline effectiveness plots are shown in Figure 25 (a-c) to compare in effectiveness at fixed length scale $\left(\Lambda_{\mathrm{x}} / \mathrm{d}_{\mathrm{m}}\right)$ and varying turbulence intensity, and Figure 25 (d-f) shows the difference in effectiveness for a fixed turbulence intensity and varying length scale for each of the nine adiabatic cases. An attempt was made to present the data in the clearest manner, allowing low turbulence intensities, $5 \%$, to be the lightest color, moderate intensities, $10 \%$, to be an intermediate color, and high turbulence intensities, $20 \%$, to be the darkest color. Length scales normalized by the main cooling hole diameter, $\Lambda_{\mathrm{x}} / \mathrm{d}_{\mathrm{m}}$, of 1,3 , and 6 are colored as blue, green, and red, respectively. It can be seen for all cases that there is no significant prediction of jet lift-off, or at least whatever lift-off there is lasts for a very short streamwise distance before re-attaching.

The centerline data starts immediately downstream of the exit of the main film cooling hole at streamwise location, $\mathrm{x} / \mathrm{d}_{\mathrm{m}}$, of $2.0{ }^{*}$ At this point the centerline film cooling effectiveness is at its peak, likely because the jet velocity is maximum here, and the jet is closes to the plate. From the maximum it steadily declines to a local minimum near an $\mathrm{x} / \mathrm{d}_{\mathrm{m}}$ of 10 (with the exception of a slight increase at $\mathrm{x} / \mathrm{d}_{\mathrm{m}}$ of approximately 3 ). This indicates that the main film cooling jet is slightly detaching from the wall but is not considered complete jet lift-off.

\footnotetext{
* The origin of the coordinate system is at the leading edge of the film cooling hole. Since the angle the main hole makes with the plate is $30^{\circ}$, the start of the data presented is at $\mathrm{x} / \mathrm{d}_{\mathrm{m}}=2.0$.
} 


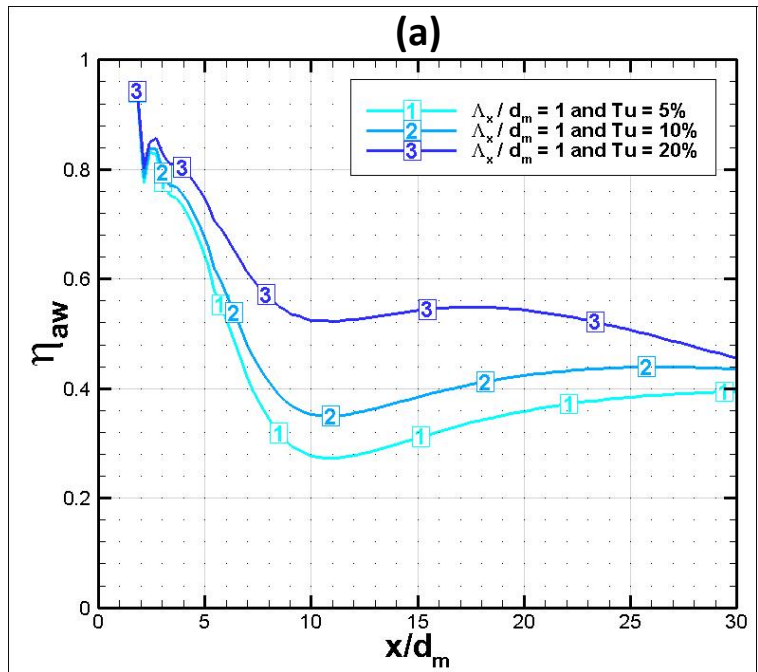

(b)

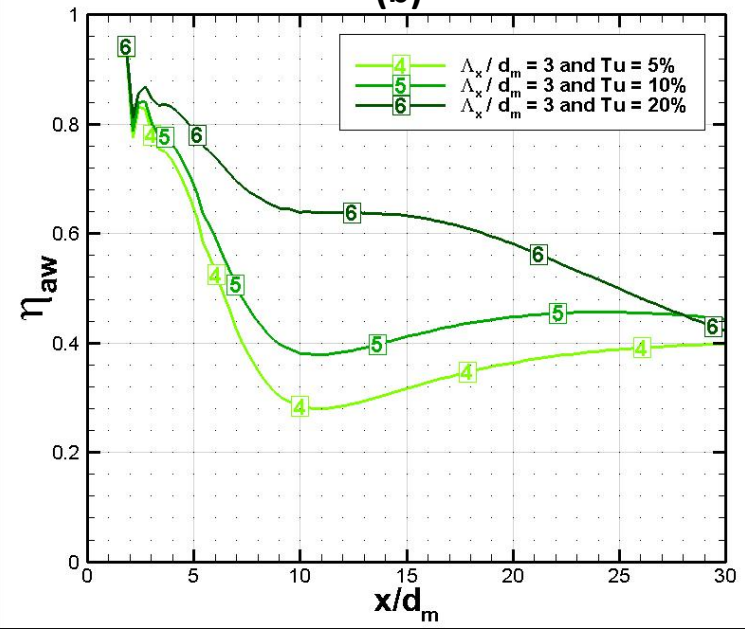

(c)

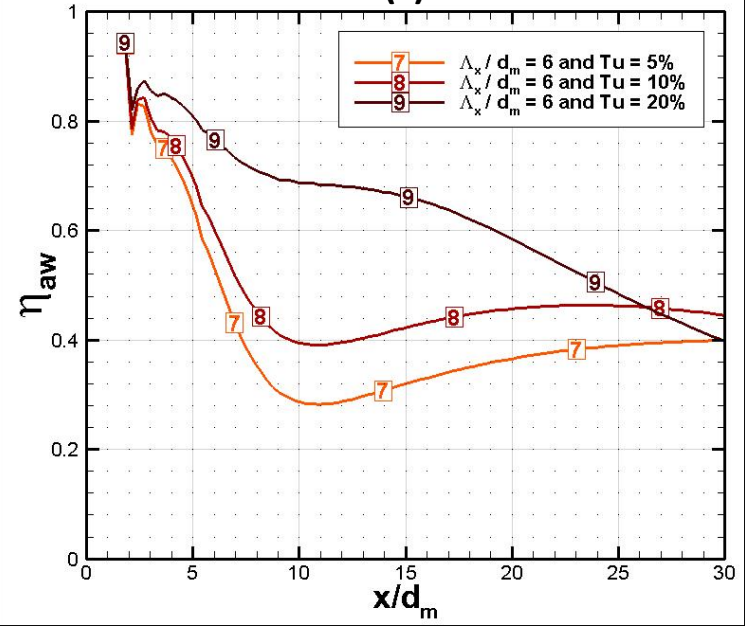

(d)

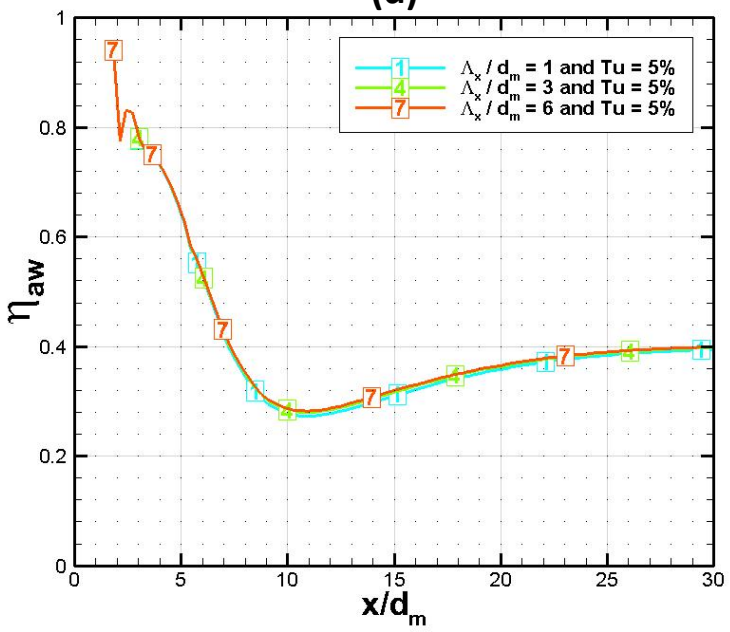

(e)

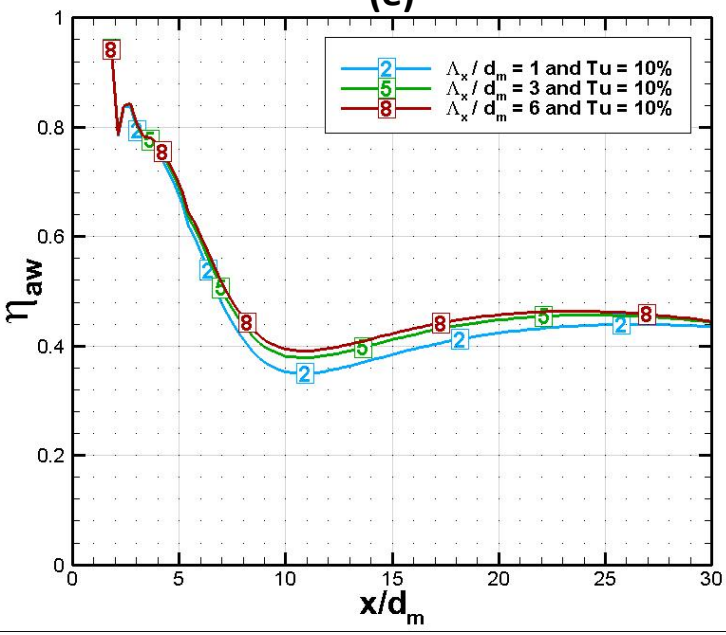

(f)

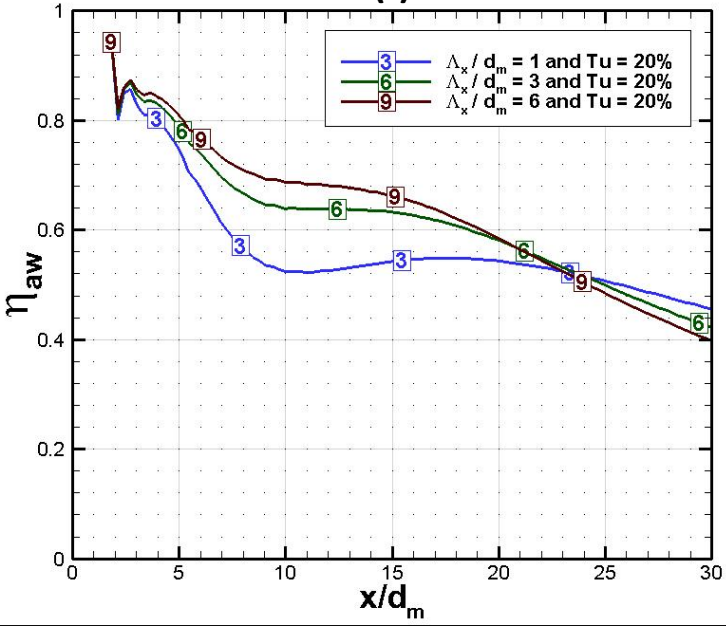

Figure 25: Centerline Adiabatic Effectiveness at Constant Length SCALE (A-C) AND CONSTANT TURBULENCE INTENSITY (D-F) 
For the higher turbulence levels the gradient is less steep and the local minima is higher indicating that the main coolant jet remains closer to the wall and better attached. Further downstream the higher turbulence levels tend to continually decrease until the end of the domain is reached, whereas the lower turbulence levels tend to rise until the end of the domain. The preliminary study found that increasing the length scale from 1 to 3 at a turbulence intensity of $10 \%$ almost uniformly decreases the centerline effectiveness at all streamwise locations. The current results found that increasing the length scale in the same manner increases the centerline effectiveness almost uniformly across the domain; but is only a relatively minor increase. At large length scales and high turbulence intensities there is sufficient mixing far downstream that the effectiveness falls near to or below that of the lower turbulence intensities, as evidenced in Figure 25(c).

\subsubsection{SPAN-AVERAGED EFFECTIVENESS}

The span-averaged film effectiveness plots were generated by averaging the effectiveness laterally over the computational domain at each streamwise location $\left(\mathrm{x} / \mathrm{d}_{\mathrm{m}}\right)$. It may be relevant to remember that the computational domain is half of what is pictured in the adiabatic effectiveness contour plots as described in section 2.2. The data was mirrored across the symmetry plane to allow for a more complete picture of the physics of the full flow while saving time by using less computational power than simulating the full span.

Span-averaged film effectiveness gives the best quantitative overall look into how well a given cooling geometry functions and, along with the qualitative results of contour plots, is the most common tool when analyzing film cooling performance. In Figure 26 the span-averaged film cooling effectiveness is given at constant length scales $\left(\Lambda_{\mathrm{x}} / \mathrm{d}_{\mathrm{m}}\right)$ of 
1, 3 and 6 in subplots (a), (b), and (c) respectively. As the turbulence intensity is increased, the span-averaged effectiveness is increased at nearly all locations in the domain, regardless of the length scale. In the case of $\Lambda_{x} / d_{m}$ of 1 , the small scale eddies do not effectively laterally mix the coolant jet as the large scale eddies do with a $\Lambda_{\mathrm{x}} / \mathrm{d}_{\mathrm{m}}$ of 6 . This can be best seen in comparing Figure 26 (a) and (c). In the current study the streamwise location of maximum spanwise averaged cooling moves upstream with increasing turbulence intensity. At the highest turbulence intensity, Figure 26 (f), the effects of turbulent length scale are apparent as it moves the point of maximum effectiveness upstream. This effect is not evident at the lower turbulence levels. For case 9, with the highest turbulence intensity and length scale, the point of maximum spanaveraged cooling is at an approximate streamwise location $\left(\mathrm{x} / \mathrm{d}_{\mathrm{m}}\right)$ of 15 . For the lowest turbulence intensity (case 1, 4, and 7) it appears that the point of maximum cooling is downstream of the domain $\left(\mathrm{x} / \mathrm{d}_{\mathrm{m}}>30\right)$. 

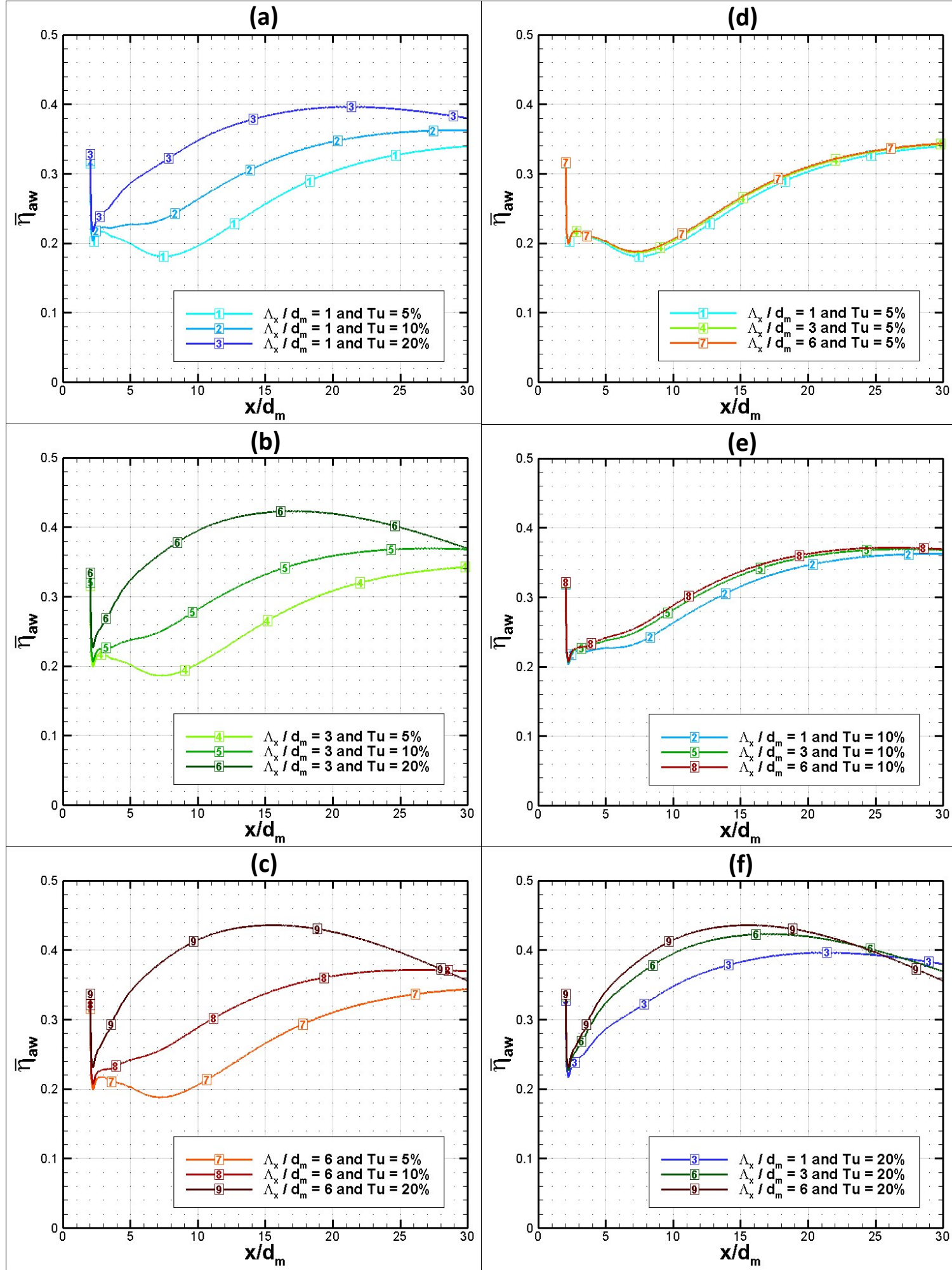

\section{Figure 26: Span-Averaged Adiabatic EfFectiveness at Constant Length SCALE (A-C) AND CONSTANT TURBULENCE INTENSITY (D-F)}




\subsubsection{AREA-AVERAGED EFFECTIVENESS}

Table 4 shows that there is a significant increase in the area-averaged effectiveness with an increase in turbulence intensity. It also shows that there is a slight but noticeable increase in the area-averaged effectiveness with increasing length scale. As a comparison to the preliminary data in [24], there is an over prediction of effectiveness which may be a result of the difference in CFD code, implementation of the turbulence model, differences in grid spacing, or a combination of all three of theses factors.

Table 4: Area-Averaged Adiabatic Effectiveness

\begin{tabular}{||l|l|l|}
\hline \multirow{2}{*}{ Case } & \multicolumn{2}{|c|}{ Area-Averaged Effectiveness } \\
\cline { 2 - 3 } & \multicolumn{1}{|c|}{ Current } & Previous [24] \\
\hline $1 . \Lambda_{\mathrm{x}} / \mathrm{d}_{\mathrm{m}}=1$ and $\mathrm{Tu}=5 \%$ & 0.2657 & 0.3411 \\
\hline 2. $\Lambda_{\mathrm{x}} / \mathrm{d}_{\mathrm{m}}=1$ and $\mathrm{Tu}=10 \%$ & 0.3079 & 0.3703 \\
\hline $3 . \Lambda_{\mathrm{x}} / \mathrm{d}_{\mathrm{m}}=1$ and $\mathrm{Tu}=20 \%$ & 0.3602 & \\
\hline $4 . \Lambda_{\mathrm{x}} / \mathrm{d}_{\mathrm{m}}=3$ and $\mathrm{Tu}=5 \%$ & 0.2706 & \\
\hline $5 . \Lambda_{\mathrm{x}} / \mathrm{d}_{\mathrm{m}}=3$ and $\mathrm{Tu}=10 \%$ & 0.3200 & 0.3786 \\
\hline $6 . \Lambda_{\mathrm{x}} / \mathrm{d}_{\mathrm{m}}=3$ and $\mathrm{Tu}=20 \%$ & 0.3848 & \\
\hline 7. $\Lambda_{\mathrm{x}} / \mathrm{d}_{\mathrm{m}}=6$ and $\mathrm{Tu}=5 \%$ & 0.2722 & \\
\hline $8 . \Lambda_{\mathrm{x}} / \mathrm{d}_{\mathrm{m}}=6$ and $\mathrm{Tu}=10 \%$ & 0.3241 & \\
\hline $9 . \Lambda_{\mathrm{x}} / \mathrm{d}_{\mathrm{m}}=6$ and $\mathrm{Tu}=20 \%$ & 0.3930 \\
\hline
\end{tabular}

\subsection{Summary of Heat Transfer Cases}

Heat transfer to a film cooled blade has been defined in equation (3-1) and is consistent with past film cooling research [44].

$$
q^{\prime \prime}=h_{f}\left(T_{a w}-T_{w}\right)
$$

This convective heat transfer coefficient is reported in the present study in its nondimensional form, $\left(\mathrm{h}_{\mathrm{f}} / \mathrm{h}_{0}\right)$, being normalized by the heat transfer coefficient at the no film cooling condition $\left(\mathrm{h}_{0}\right)$ as is common in most film cooling studies. Obtaining the heat transfer coefficient, $\mathrm{h}_{\mathrm{f}}$, required two CFD simulations at the same flow conditions with different heat fluxes at the wall to be processed simultaneously. The first simulation had 
an adiabatic condition at the walls and in the second a nonzero, specified heat flux was used. The two temperatures in equation (3-1), $\mathrm{T}_{\mathrm{aw}}$ and $\mathrm{T}_{\mathrm{w}}$, are the temperatures at the wall for the adiabatic and specified heat flux respectively. Along with the two film cooling simulations, a third flat plate study was used to determine the heat transfer coefficient over a flat plate and compared to existing Nusselt number correlations as has been detailed in section 2.2.2.

Sen et al. [45] introduced the net heat flux reduction (NHFR) as a parameter to determine net benefit from film cooling. The NHFR is defined in equation (3-2). The NHFR serves to quantify the relationship between the heat transfer coefficient and the adiabatic effectiveness.

$$
N H F R=1-\frac{q^{\prime \prime}}{q_{0}^{\prime \prime}}=1-\frac{h_{f}\left(T_{a w}-T_{w}\right)}{h_{0}\left(T_{\infty}-T_{w}\right)}
$$

The primary purpose behind film cooling is to decrease both the dimensionless heat transfer coefficient $\left(\mathrm{h}_{\mathrm{f}} / \mathrm{h}_{0}\right)$ and the adiabatic wall temperature $\left(\mathrm{T}_{\mathrm{aw}}\right)$. A dimensionless temperature is defined in equation (3-3) and is used to reduce the NHFR equation to its most useful form in equation (3-4). The dimensionless temperature is generally assumed to be a constant so that the NHFR can be solved for directly. In this study a dimensionless temperature of 1.6 is used and considered representative of engine conditions [13].

$$
\begin{gathered}
\theta=\frac{T_{\infty}-T_{c}}{T_{\infty}-T_{w}} \\
N H F R=1-\frac{h_{f}}{h_{0}}\left(1-\eta_{a w} \theta\right)
\end{gathered}
$$


The range for NHFR is typically between zero and one. The NHFR can be less than zero if there is a net heat flux gain with the introduction of film cooling. This is possible in certain areas, such as in the near hole region, where the heat transfer coefficients can be high. This phenomenon was studied by Goldstein and Taylor [46] through the heat-mass transfer analogy for a cylindrical hole.

The dimensionless heat transfer coefficient and the NHFR are presented in the same way as the adiabatic effectiveness with the exclusion of the centerline data and contour plots. Centerline data for the heat transfer coefficient and NHFR is not considered to be a useful representation of the flow physics and is omitted for that reason.

\subsubsection{SPAN-AVERAGED HEAT TRANSFER}

The span-averaging calculation for the dimensionless heat transfer coefficient and NHFR was obtained in the same manner as the effectiveness. The dimensionless heat transfer was shown to reach a maximum within 5 hole diameters downstream of the cooling holes in all cases. High turbulence intensity tends to increase the peak of the maximum heat transfer coefficient downstream of the hole, but the location remains the same. This peak in heat transfer coefficient is visible in the dimensionless heat transfer coefficient plots shown in Figure 27. There is a secondary peak in the heat transfer around 4-7 hole diameters downstream that is of interest in the lower turbulence cases. This secondary peak disappears at high turbulence and large length scale cases (Case 6 and 9). The secondary peak in heat transfer occurs at or near the minimum of the NHFR in all cases. The location of the secondary peak was taken into account when analyzing the contours of the NHFR, and it was found that the area between the holes could be an issue in the cooling. The high turbulence case reaches a peak near a streamwise location 
$\left(\mathrm{x} / \mathrm{d}_{\mathrm{m}}\right)$ of 15 and then declines gradually moving away from the cooling holes. The low turbulence case tends to reach a minimum at the same location as the secondary peak in heat transfer coefficient and rises gradually for the rest of the domain.

Overall, the increases in the heat transfer coefficient do not degrade the performance of the AVH enough to overshadow the adiabatic effectiveness. The highest turbulence intensity and length scale case has the highest NHFR with a maximum value near 15 hole diameters downstream of the cooling holes. This corresponds to the same location of maximum cooling as found in the adiabatic effectiveness of section 3.1.3. As in the case of the adiabatic effectiveness, turbulence intensity and length scale tends to increase the NHFR and thus the cooling performance of the AVH design. Length scale is shown in the heat transfer cases to have little effect at low turbulence levels and a somewhat larger effect as the turbulence intensity is increased. 


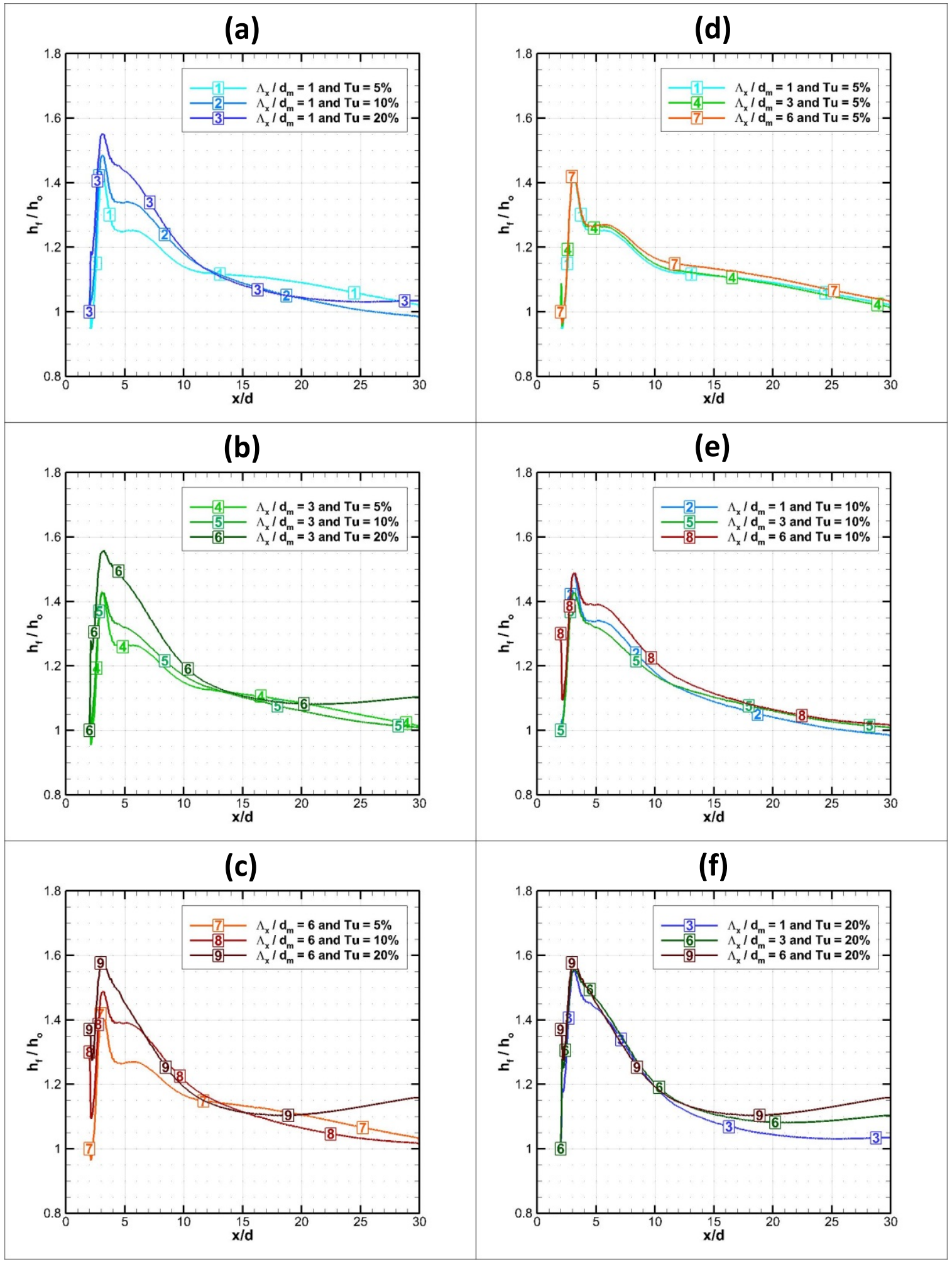

Figure 27: Span-Averaged Dimensionless Heat Transfer Coefficients at Constant Length Scale (A-C) And Constant Turbulence Intensity (D-F) 


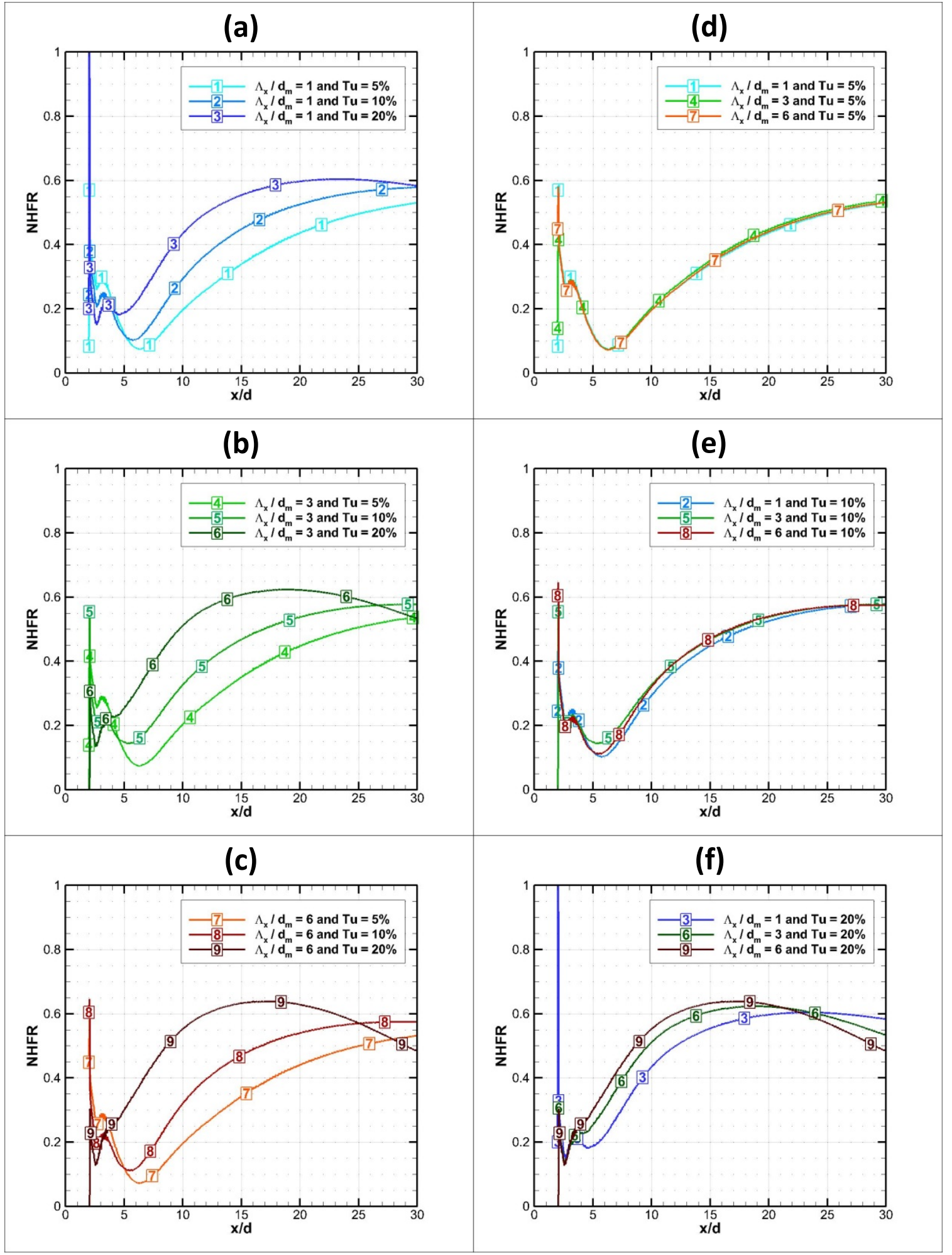

Figure 28: Span-Averaged NHFR at Constant Length Scale (A-C) ANd CONSTANT TURBULENCE INTENSITY (D-F) 


\subsubsection{AREA-AVERAGED HEAT TRANSFER}

The area-averaged values are beneficial in the fact that one can get a generalized idea of the impact of the elevated turbulence intensities and length scales. Depicted in Table 5, as the turbulence intensity is increased, the NHFR is noticeably increased as well. Conversely, increasing the turbulent length scale has shown a much smaller effect on the NHFR at the lower length scales but does in fact have a more discernable effect in increasing NHFR at larger length scales.

When examining the dimensionless heat transfer coefficient, an increase in turbulence results in only a minor increase of the nondimensional heat transfer coefficient. The length scale has limited effects on the nondimensional heat transfer coefficient, but once again has a more discernable effect in increasing the heat transfer coefficient at larger length scales. The area-averaged heat transfer coefficient and NHFR are broad measurements of a highly localized phenomenon. It must be understood that this localized phenomena cannot capture the detail through the area-averaging process. It is important to reiterate that the increase in heat transfer coefficient due to film cooling being present does not over shadow the adiabatic effectiveness. The net benefit provided by the cooling holes is best quantified by the NHFR.

Table 5: Area-Averaged Heat Transfer

\begin{tabular}{|l|c|c|c|c||}
\hline \multicolumn{1}{|c|}{ Case } & $h / h_{0}$ & $h / h_{0}$ & $N H F R$ & $N H F R$ \\
\hline $1 . \Lambda_{\mathrm{x}} / \mathrm{d}_{\mathrm{m}}=1$ and $\mathrm{Tu}=5 \%$ & 1.123 & 1.3672 & 0.3415 & 0.3790 \\
\hline $2 . \Lambda_{\mathrm{x}} / \mathrm{d}_{\mathrm{m}}=1$ and $\mathrm{Tu}=10 \%$ & 1.121 & 1.2542 & 0.4098 & 0.4889 \\
\hline $3 . \Lambda_{\mathrm{x}} / \mathrm{d}_{\mathrm{m}}=1$ and $\mathrm{Tu}=20 \%$ & 1.147 & & 0.4845 & \\
\hline $4 . \Lambda_{\mathrm{x}} / \mathrm{d}_{\mathrm{m}}=3$ and $\mathrm{Tu}=5 \%$ & 1.124 & & 0.3487 & \\
\hline $5 . \Lambda_{\mathrm{x}} / \mathrm{d}_{\mathrm{m}}=3$ and $\mathrm{Tu}=10 \%$ & 1.125 & 1.2603 & 0.4260 & 0.5032 \\
\hline $6 . \Lambda_{\mathrm{x}} / \mathrm{d}_{\mathrm{m}}=3$ and $\mathrm{Tu}=20 \%$ & 1.182 & & 0.5127 & \\
\hline $7 . \Lambda_{\mathrm{x}} / \mathrm{d}_{\mathrm{m}}=6$ and $\mathrm{Tu}=5 \%$ & 1.141 & & 0.3429 & \\
\hline $8 . \Lambda_{\mathrm{x}} / \mathrm{d}_{\mathrm{m}}=6$ and $\mathrm{Tu}=10 \%$ & 1.151 & & 0.4219 & \\
\hline $9 . \Lambda_{\mathrm{x}} / \mathrm{d}_{\mathrm{m}}=6$ and $\mathrm{Tu}=20 \%$ & 1.198 & & 0.5240 & \\
\hline
\end{tabular}




\subsection{COMPARISON OF URANS AND RANS}

For a cylinder in a cross flow a well known and characterized Von-Karman vortex street exists downstream of the cylinder. In a film cooling flow the coolant jet has sufficient momentum when ejecting into the main stream at high blowing ratios and can be considered to be loosely analogous to a cylinder in a cross flow. The interaction of the cylindrical coolant jet develops eddies downstream of the coolant hole similar to the Von-Karman vortex street. With this inherently unsteady interaction between the coolant jet and main stream an unsteady analysis is necessary to better understand the performance of film cooling. Since the AVH concept was designed to mitigate the CRV, unsteady vortical interaction in the main stream may be important to analyze as well.

Steady RANS coupled with an appropriate turbulence model is a valid first approximation to understanding the film cooling jet interaction with the main stream. Harrison and Bogard [35] studied the prediction of numerous turbulence models on straight film cooling holes and determined that the prediction of adiabatic wall temperatures can vary between models. Voigt et al. [47] was able to show a better matching to experimental data with a URANS approach than with a steady RANS approach. Thus, URANS is the logical next step in analyzing the film cooling performance. By using a URANS model, the highly unsteady, three-dimensional nature of film cooling flow can be better understood. URANS is limited by the fact that it only captures the fluctuations in the mean quantities of the flow and still requires a turbulence closure model. In section 3.1 and 3.2 it was noted that the effect of length scale on the film cooling performance was much smaller than that of the turbulence intensity. This allowed the unsteady cases for the AVH to be limited to three different turbulence 
intensities $(5,10$ and $20 \%)$ with a normalized length scale, $\Lambda_{x} / \mathrm{d}_{\mathrm{m}}$, of 1 . Computational time for a URANS simulation for these three unsteady cases was nearly 2 orders of magnitude longer than the nine steady RANS case. This can be attributed to the number of inner iterations needed per each time step and the overall number of time steps needed. The time to calculate each iteration is also longer due to the increased size of the domain and the increase in the number of cells. Reported figures and data for the URANS simulations began after at least 1000 time steps to make sure there is little influence of the steady starting point of the unsteady simulation.

\subsubsection{SECONDARY VELOCITY VECTORS DOWNSTREAM OF COOLING HOLES}

Downstream of the hole it is helpful to look at the velocity tangential to the freestream flow to visualize the vorticity generated by interaction of the coolant with the freestream. The amount of upwash created by the side holes is easily visible when looking at the secondary velocity vectors at discrete streamwise locations. Figure 29 and Figure 30 show the velocity vectors colored by the magnitude of the vectors at four discrete streamwise locations for the $20 \%$ turbulence case with $\Lambda_{\mathrm{x}} / \mathrm{d}_{\mathrm{m}}$ of 1 . The $20 \%$ turbulence case is highlighted because it shows the most drastic changes farther away from the hole. On the left hand side of the symmetry line is the results of the steady RANS simulation, which served as the initial conditions for the unsteady RANS simulation. The right hand side is the quasi-instantaneous results from the unsteady RANS after more than 3000 time steps.

It is readily apparent that moving further away from the cooling holes the vorticity is decreased greatly in the URANS simulation. 


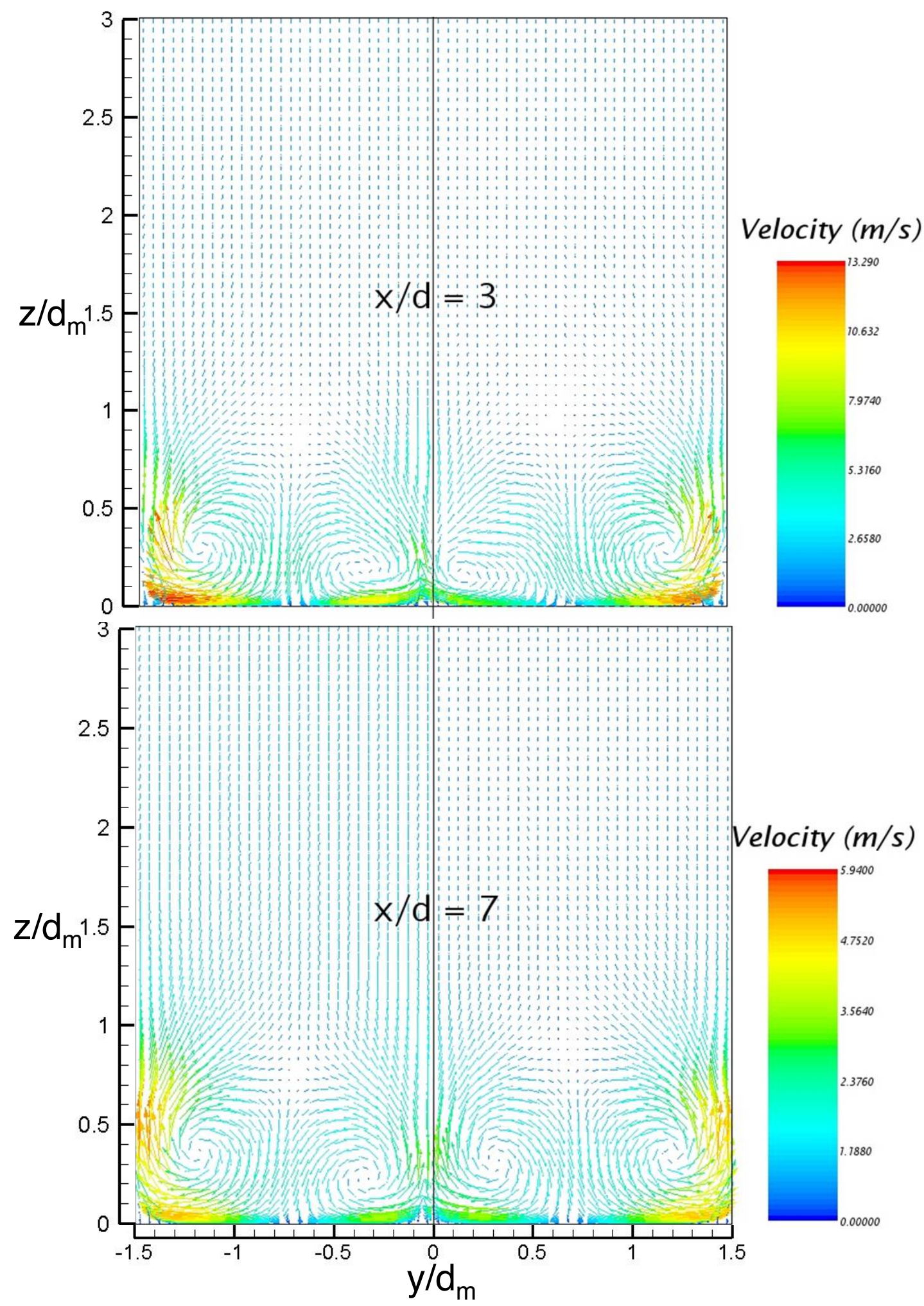

Figure 29: Velocity Vectors TANGential to the FreEstream AT $\mathrm{X} / \mathrm{d}_{\mathrm{m}}=3$ AND $X / d_{m}=7$ (RANS - RIGHT, URANS - LEFT, TU $=20 \%$, AND $\left.\Lambda_{\mathrm{X}} / \mathrm{d}_{\mathrm{m}}=1\right)$ 


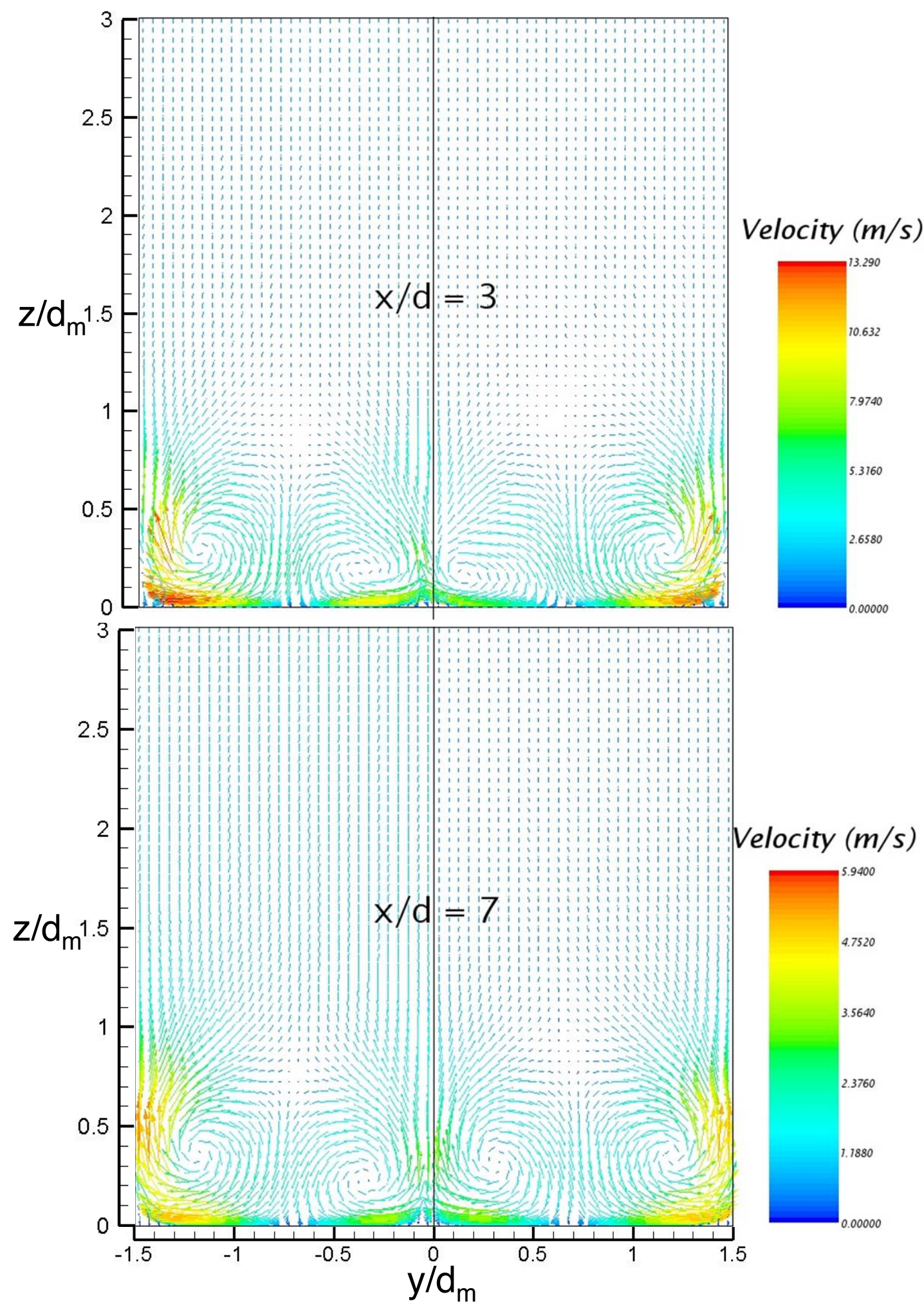

Figure 30: Velocity Vectors Tangential to the Freestream AT $\mathrm{X} / \mathrm{d}_{\mathrm{m}}=\mathbf{1 5}$ AND $X / d_{m}=25\left(\right.$ RANS - RIGHT, URANS - LEFT, TU $\left.=20 \%, A N D \Lambda_{X} / d_{m}=1\right)$ 
Near to the hole the upwash produced by the side holes appears to be stronger than that of the main cooling hole. This strong upwash is balanced by creating a downwash on the main cooling hole and promoting a better attached cooling layer. Three normalized hole diameters downstream of the main cooling hole for the $20 \%$ turbulence case is a location of interest because of the increased heat transfer to the wall between the holes. This location was found to have a nearly zero NHFR with local cells being slightly less than zero. This region has been highlighted in Figure 31.

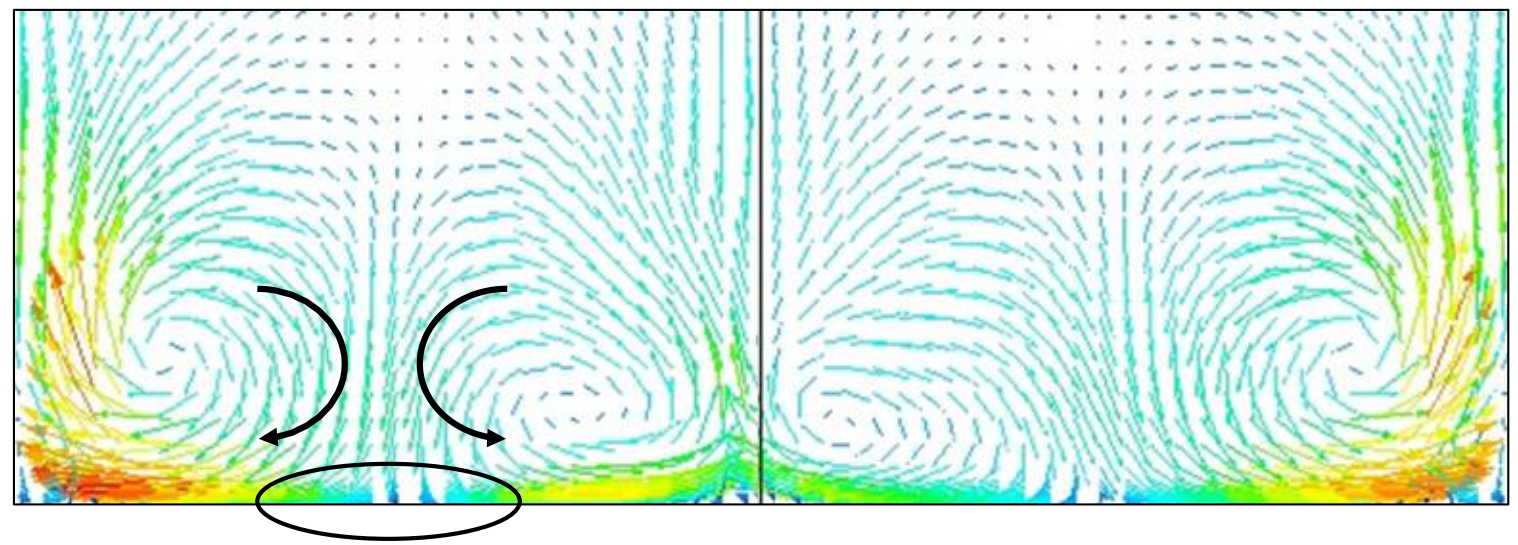

FiguRE 31: VELOCITY VECTOR AT X/D =3 SHOWING REGION OF INCREASED HEAT Transfer BetWeen Holes (URANS ONLY)

It can be seen from Figure 29 and Figure 30 that there is little difference between the RANS and URANS velocity fields. The primary difference in the velocity fields was found to be the pulsating nature of the coolant jet that was found in the unsteady cases. This led to the changes in the temperature at the wall and along the hole centerline as discussed in section 3.3.3.

\subsubsection{UNSTEADINESS IN THE WAKE OF THE MAIN COOLING JET}

The adiabatic film cooling effectiveness contours behind the main film cooling jet for the steady RANS and URANS are shown in Figure 32. The steady case shows 3 distinct streamlined cooling trails downstream of the anti-vortex holes that begin to spread 
laterally around 10 hole diameters downstream for the low turbulence case. It can be seen in the unsteady case that eddy-like structures develop from the interaction of the main stream with the main cooling jet downstream of the cooling holes. These structures develop and convect downstream at a constant frequency in the simulation. This unsteadiness in the main cooling hole is a primary reason behind the differences between the RANS and URANS temperature predictions at the wall. This unsteadiness is minor, but lower surface temperatures predicted by the URANS could be important. Experimental validation and high fidelity LES may be important to determine whether the RANS or URANS surface temperature prediction at the wall is more accurate, and whether a URANS simulations is important in order to obtain the best accuracy.

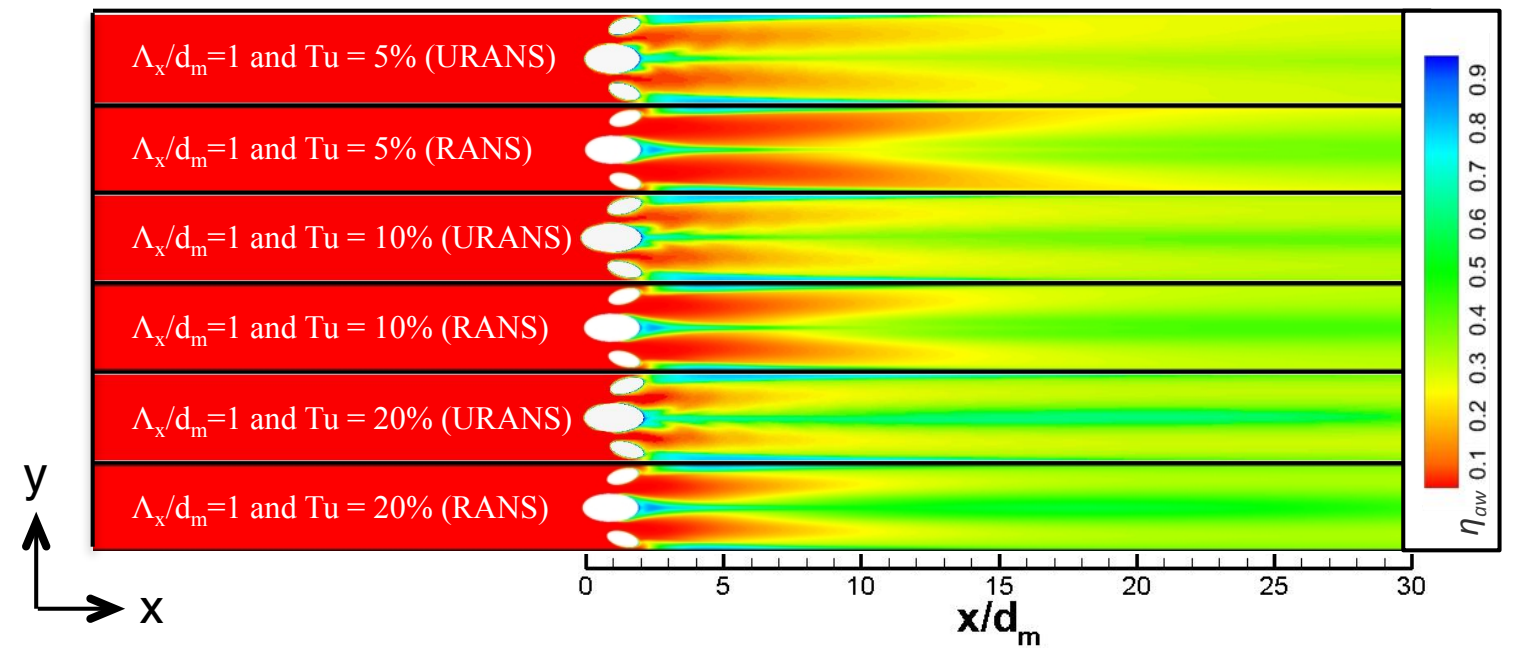

Figure 32 Comparison of RANS ANd URANS PREdictions of Adiabatic FILM COOLING EFFECTIVENESS

The analysis of the unsteady cases compared to steady cases tends to reinforce the trend of increasing cooling effectiveness with the increasing turbulence intensity. There is some slight difference in the magnitude and shape of the cooling trails as can be seen in Figure 32. This may lie in the difference in grid spacing off the wall and in the near wall region. Qualitative analysis of the difference between the URANS instantaneous and 
averaged values is shown in Figure 33. The averaged URANS values were calculated using an ensemble average across timesteps 1000 to 3000 . The absolute difference was taken between the quasi-instantaneous URANS and the averaged URANS. The scale was truncated at \pm .01 and filled in an attempt to wash out any interpolation errors from interpolating from the mesh to the grid that was used to post process the values. A vortex shedding exists in the wake of the cooling holes with alternating cool and hot spots being apparent in the surface temperatures. The alternating cool and hot spots convect downstream at the same frequency as the pulses from the main coolant jet. The vortex shedding is washed out as the flow convects downstream and is almost completely gone 15 diameters away from the cooling holes.

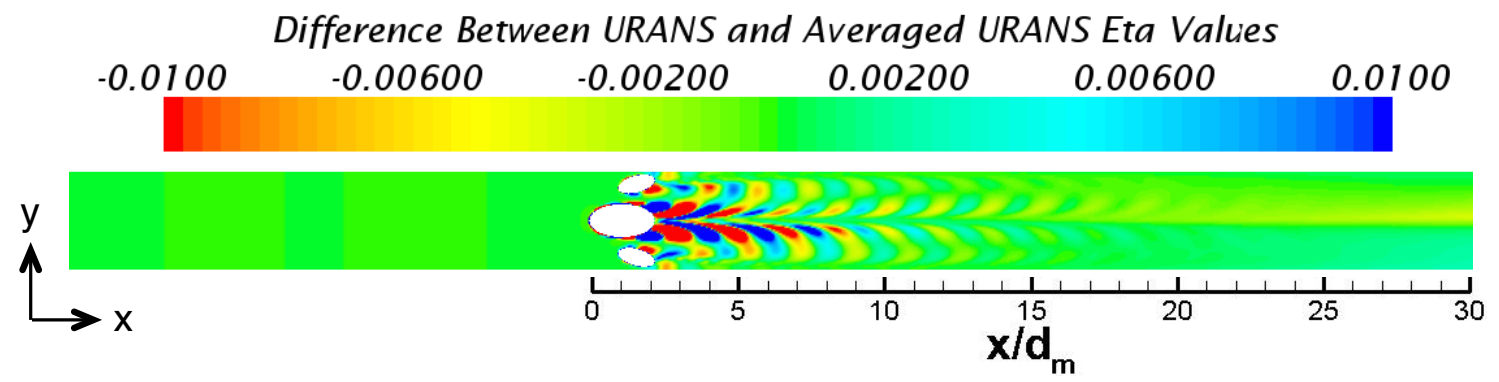

\section{Figure 33: Absolute Difference Between Quasi-Instantaneous URANS} ANd Time AVERAged URANS Film EfFectiveness $\left(T \mathrm{~T}=10 \%, \Lambda_{\mathrm{x}} / \mathrm{d}_{\mathrm{m}}=1\right)$

The source of the unsteadiness in the main cooling jet appears to come from the recirculation region that exists in the main cooling hole. This recirculation and low momentum region has been studied by numerous research groups including Leylek et al. [48]. This low momentum region is shown in Figure 34. Vortices created in the region are shed off and convect downstream causing the rippling in the coolant jet. Figure 35 shows four line integral convolution of the velocity vectors at a plane along the main hole centerline at different times, where the black box highlights a single eddies evolution as it convects downstream. The center of a single eddy is shown as a white circle. In this 
figure the flow is displayed with a contour colored by the velocity magnitude tangential to the plane. The recirculation region can be influenced by the plenum conditions in a real engine. In a real engine there are cross flows that can affect the size and shape of the low momentum region as well as the location of the separation. This may affect the unsteadiness in the main film cooling jet and may be something to consider in more detail in future work.

To better study the effect of these eddies on the coolant flow an isosurface was created with a dimensionless temperature $(\Theta)$ of 0.2 in all of the cooling flows. The dimensionless temperature is defined in equation 3.5.

$$
\Theta=\frac{T_{\infty}-T}{T_{\infty}-T_{C}}
$$

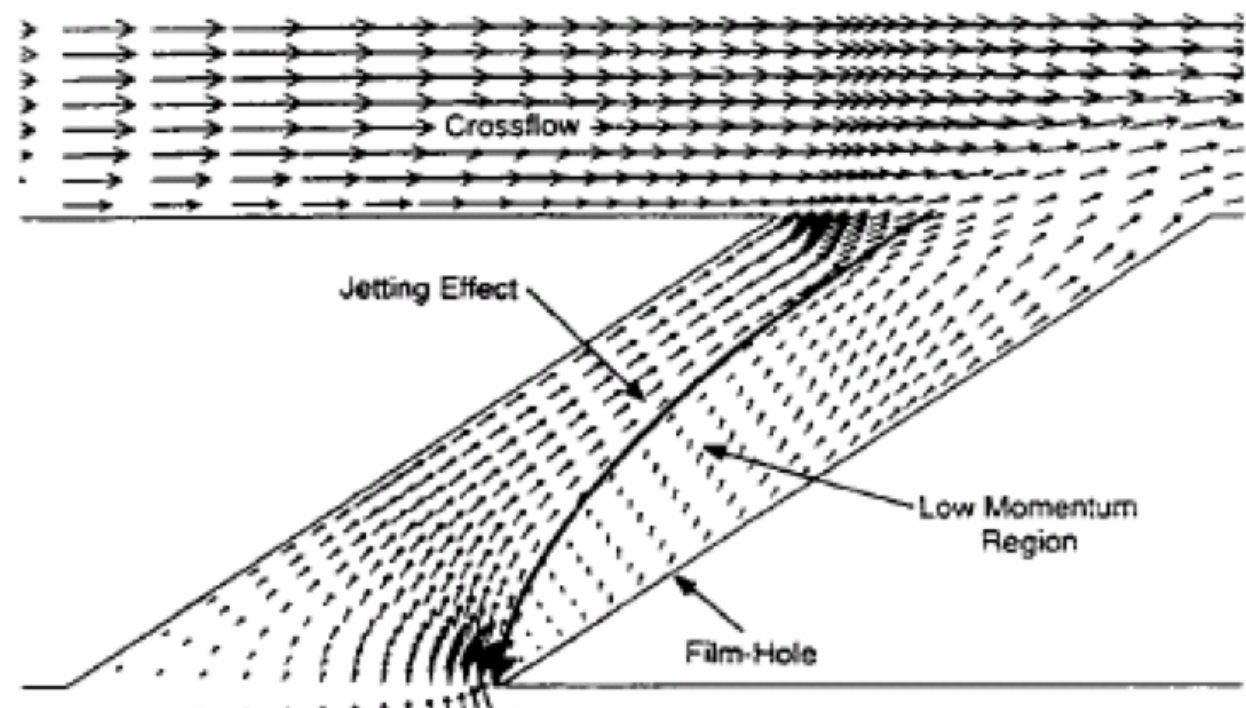

Figure 34: Velocity Vectors Inside of a Film Cooling Hole With Overlaid Schematic Showing Area of Recirculation Leylek et al. [48] 


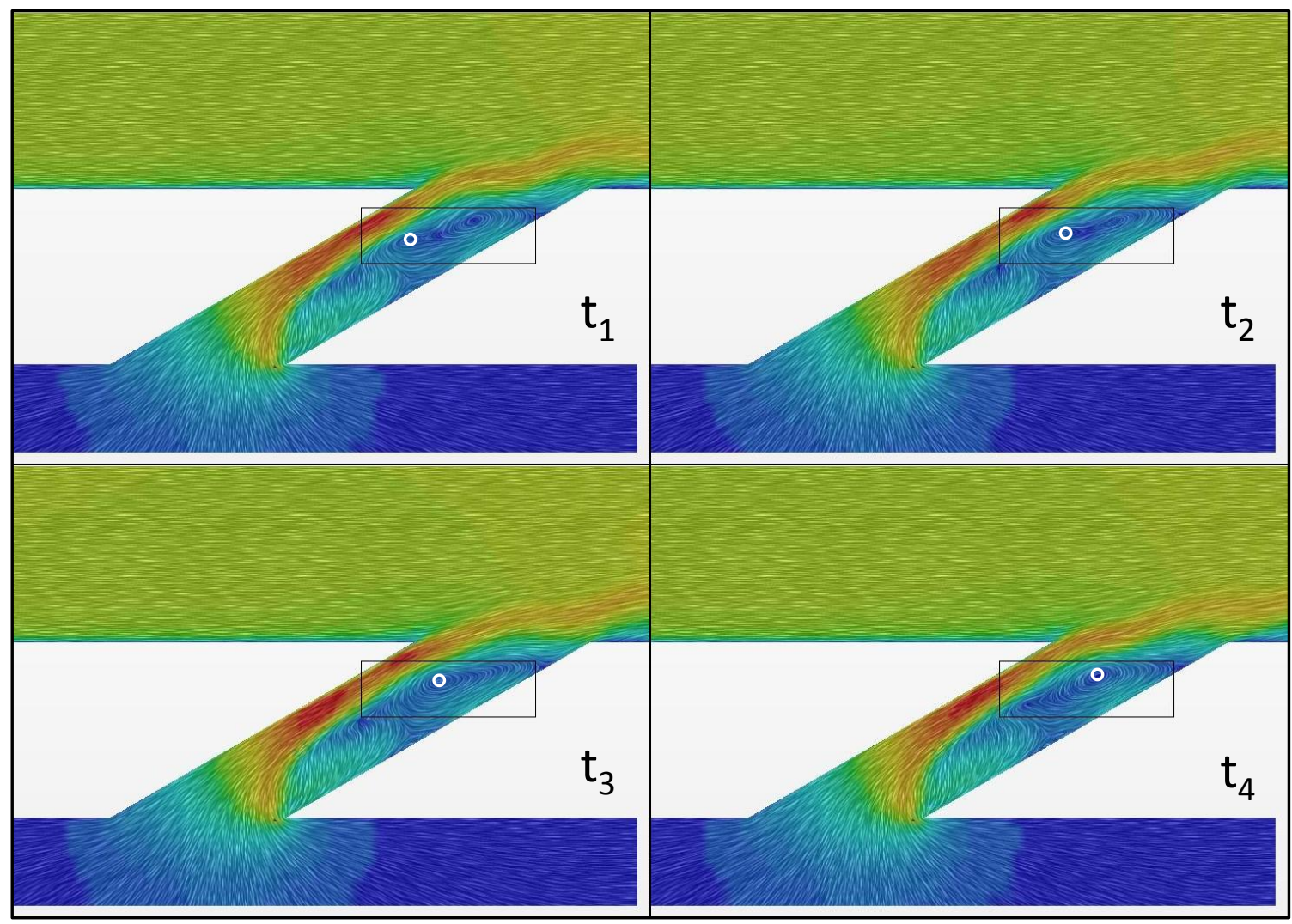

\section{Figure 35: Line Integral Convolution of Main Hole Centerline at Four Time Levels Showing Pulsating Flow $\left(\mathrm{Tu}=\mathbf{2 0} \%, \Lambda_{\mathrm{X}} / \mathbf{d}_{\mathrm{m}}=1\right)$}

Figure 36 shows four frames of this isosurface contour for the middle turbulence level $\left(\mathrm{Tu}=10 \%, \Lambda_{\mathrm{x}} / \mathrm{d}_{\mathrm{m}}=1\right)$. From this it can be seen how the three coolant jets are mixing downstream and the effect from the eddies caused in the main coolant hole. A small black box is shown to highlight a region that is geometrically fixed in space in the frames initially enclosing a region of mixing of the jets. The black box is used as a frame of reference in an attempt to show the convection of the coolant jet downstream in four discrete images. This is best seen in time-lapse animation of hundreds of images. 


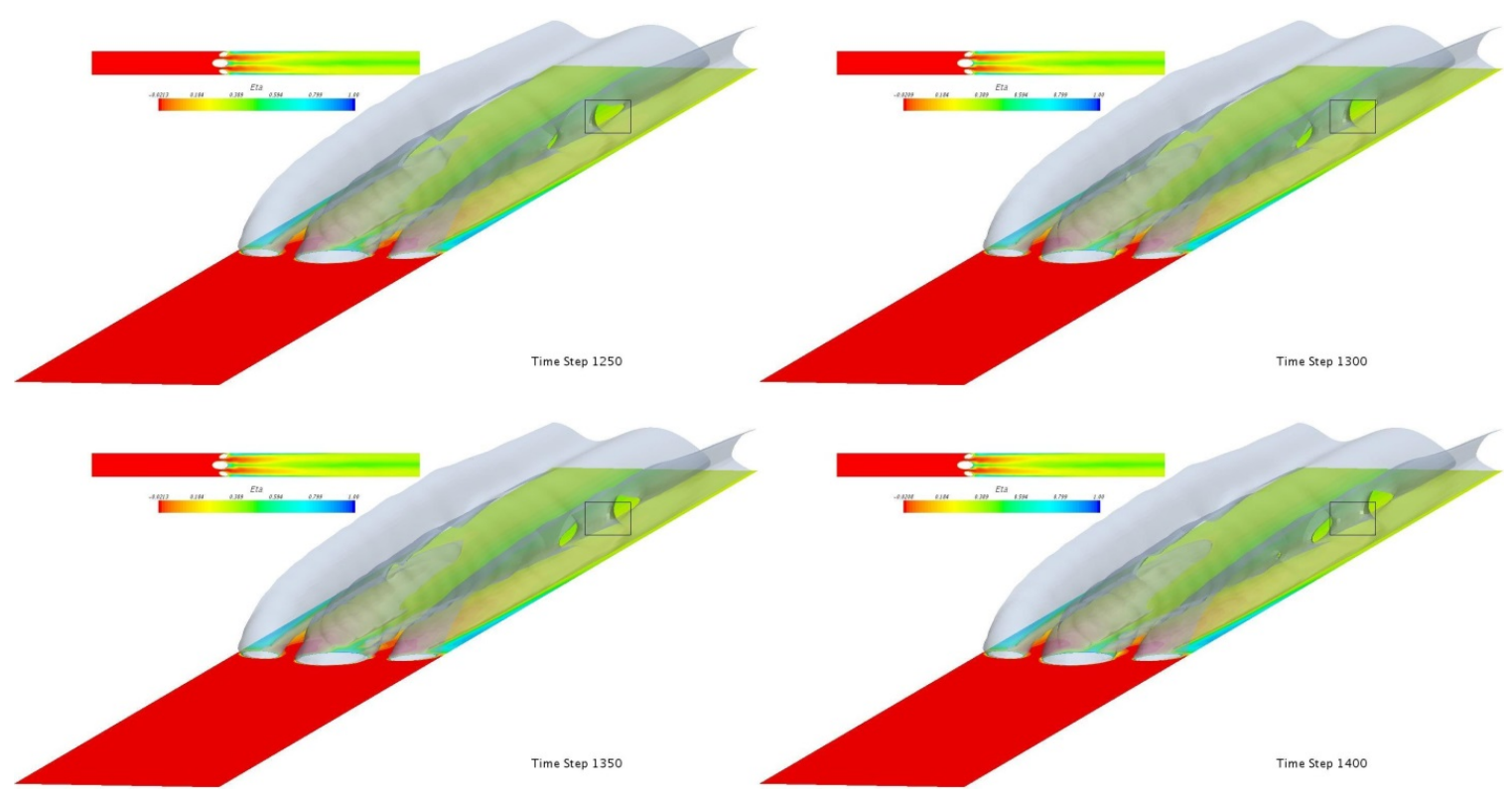

Figure 36: Isosurface of Temperature Showing Mixing of Coolant Jets For A TURbulence Intensity OF 10\% at 50 Time STEP INTERVALS

\subsubsection{COMPARISON OF URANS AND RANS TEMPERATURE FIELD PREDICTION}

In the unsteady analysis it was found that there was a slightly greater prediction of lift off for the URANS simulations along the main coolant jet compared to the steady case. Along the main hole centerline the nondimensionalized temperature field plotted in a vertical plane is shown in Figure 37. There is a slight but noticeable increase in the jet lift off for the URANS when comparing the RANS on the top to the averaged URANS in the middle. The coolant jet appears to follow a more pronounced parabolic trajectory in the URANS case than in the RANS case. This is highlighted in the region enclosed by identically sized and positioned black ovals in Figure 37. In the URANS case the coolant is able to penetrate further into the main stream than in the case for the steady analysis. An example of the instantaneous URANS is shown at the bottom of the figure for a qualitative view of the instantaneous behavior of the main coolant jet as a comparison to the averaged URANS and RANS dimensionless temperature field. 


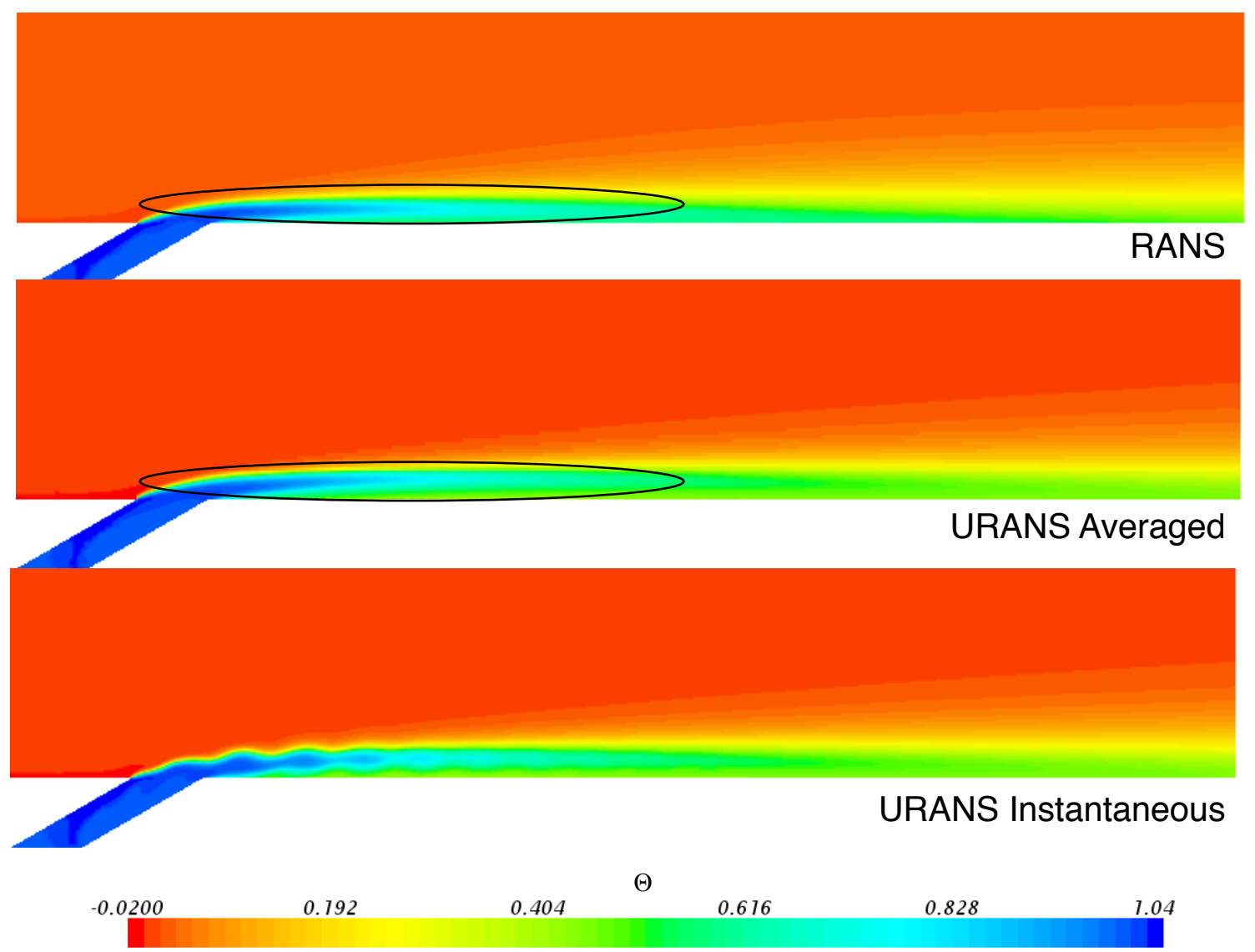

\section{Figure 37: Dimensionless Temperature Along Main Hole Centerline $\left(\mathrm{TU}=10 \%, \Lambda_{\mathrm{X}} / \mathrm{d}_{\mathrm{m}}=1\right)$}

In the mesh for the URANS cases, there was a less refined mesh near to the wall than in the RANS cases. The wall $\mathrm{y}+$ values for the URANS cases were near 1.0 while the RANS cases wall $y+$ values were an order of magnitude lower. This may result in some of the differences in magnitude of the prediction of the temperatures at the surface. The trends remain the same as well as the shape and structure of the coolant jets. As a way to quantify the decrease in jet lift off as predicted by URANS, Figure 38 is presented. The span-averaged film cooling effectiveness for the middle unsteady turbulence case shown in Figure $38\left(\mathrm{Tu}=10 \%, \Lambda_{\mathrm{x}} / \mathrm{d}_{\mathrm{m}}=1\right)$ is presented for the RANS starting point, the time average of the URANS simulation, and one arbitrary time step of the URANS simulation. 


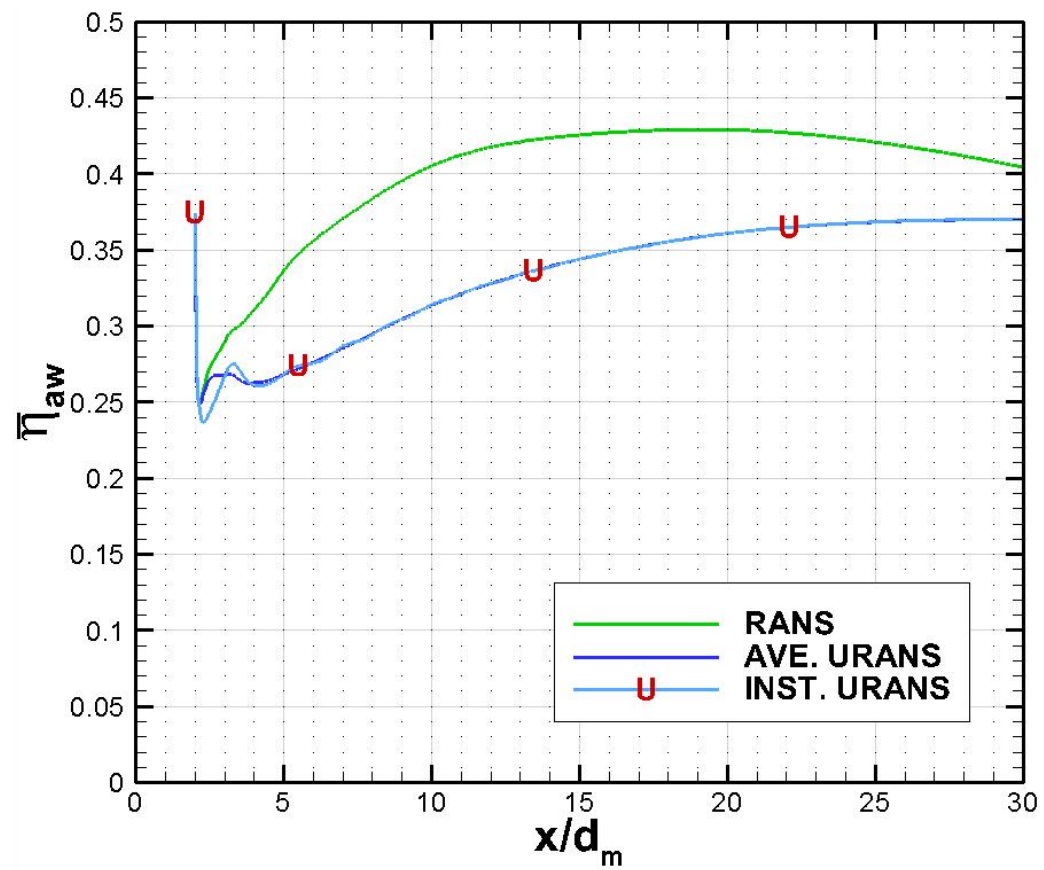

Figure 38: CoMparison OF SPAN-AVERAGED FILM EFFECTIVENESS FOR RANS AND URANS $\left(T U=10 \%, \Lambda_{\mathrm{X}} / \mathrm{d}_{\mathrm{m}}=1\right)$

It is clear that there is an increase in the prediction of the temperature at the wall for the RANS case resulting in the decrease of the adiabatic effectiveness. This also corroborates the observation that there is an increased prediction of lift off compared to the RANS case.

Only one case is presented in this manner due to problems in post processing the temperature histories output by Star-CCM+. The number of time steps and size of the mesh led to a rapidly growing file size that reached 800 gigabytes or more leading to the high and low turbulence cases to become corrupted and the temperature time histories became irretrievable. The middle turbulence case was able to be salvaged since a different output method was used after the realization that the other two histories were corrupted. 


\subsubsection{MIXING OF JETS, JET LIFT OFF, AND LATERAL SPREADING}

The mixing of the coolant streams can be visualized by way of the same isosurfaces described in section 3.3.2. The quasi-instantaneous dimensionless temperature isosurfaces of the three unsteady cases are shown in Figure 39. It is clearly be seen that the film layer becomes more attached to the wall as the turbulence intensity is increased. In the lowest turbulence case the main and secondary jets are able to penetrate further into the mainstream flow than the higher turbulence cases. The lowest turbulence cases also have the worst lateral spreading of the coolant, whereas the highest turbulence intensity has the best lateral spreading of the coolant. The pulses stemming from the recirculation region in the main cooling hole are apparent when looking at the quasiinstantaneous isosurfaces. These pulses are more distinct in the lowest turbulence case and they propagate further downstream before being damped out. At the highest turbulence intensity the coolant from the main or side holes does not penetrate as well into the freestream as compared to the low turbulence case. The highest turbulence case shows the best attachment of the coolant to the surface and the best overall coverage downstream of the cooling holes. This qualitatively confirms the results from sections 3.1 and 3.2. 

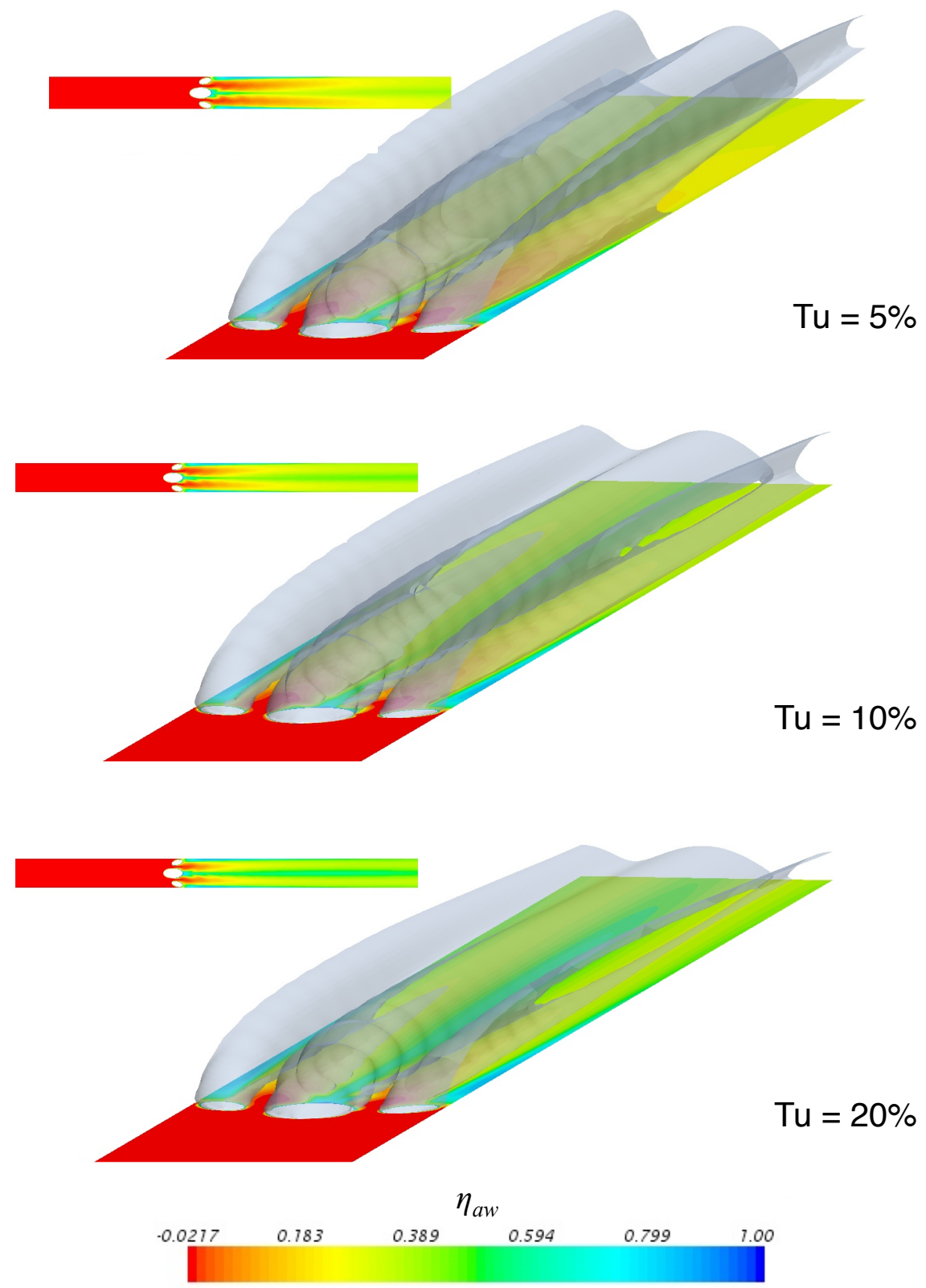

Figure 39: IsOSURFACE OF DIMENSIONLESS TEMPERATURE FOR URANS Simulations at TurbulenCe InTENSITIES OF $5 \%, 10 \%$, AND $20 \%\left(\Lambda_{\mathrm{X}} / \mathrm{d}_{\mathrm{m}}=1\right)$ 


\section{SUMMARY AND CONCLUSIONS}

\subsection{SUMMARY}

A detailed analysis examining the effect of elevated turbulence and increased turbulent length scale on the performance of a single geometry of the anti-vortex hole film cooling concept has been reported. It was found that, at high blowing ratios, and high density ratios, higher freestream turbulence levels increase the film cooling effectiveness of the AVH geometry. Although there are disparities in the data compared to the previous study, the trends and overall magnitudes remain the same at the same blowing ratio and density ratio. High freestream turbulence does not appear to have any negative effects on the film cooling effectiveness of the AVH geometry at high blowing ratio and density ratio. Unsteady results were used as a qualitative comparison tool and were able to reinforce these findings. The AVH geometry has been shown to have a substantial advantage when compared to the baseline cylindrical case from the previous study and work by Heidmann et al. [12, 13], Hunley et al. [24] and Dhungel et al.[11].

The elevated level of freestream turbulence was found to increase the span-averaged, centerline, and area-averaged film cooling effectiveness for the AVH geometry. The increase in effectiveness coverage extends approximately 30 cooling hole diameters 
downstream of the beginning of the main film cooling hole in the majority of the cases. It is interesting to note that at large length scales and high turbulence intensities there is sufficient mixing far downstream that the effectiveness falls below that of the lower turbulence intensities. Although the heat transfer coefficients are increased with increasing turbulence intensity, it is not to an extent that would decrease the benefit of using the AVH geometry. As a general trend, the NHFR is increased with turbulence intensity in all cases. Two main conclusions can be drawn from the current study:

- It has been shown that the turbulence in the hot gases exiting the combustor can aid in the film cooling for the AVH geometry at realistic blowing ratios. Heat transfer coefficients were considered but not shown to have enough of an increase to outweigh the benefit of using the AVH.

- Length scale was shown to have little to no effect at the low turbulence level and a small, but a noticeable effect at high turbulence intensity. This effect may become more pronounced with non-dimensional length scales $\left(\Lambda_{\mathrm{x}} / \mathrm{d}_{\mathrm{m}}\right)$ closer to engine representative values.

As discussed, future work can investigate further details of the flow physics through further numerical and experimental analysis, with the inclusion of more test parameters including approaching boundary layer profiles to the holes and additional blowing ratios and density ratios. 


\subsection{Application to Gas Turbine Design}

Implementation of the $\mathrm{AVH}$ concept to real gas turbine engines could lead to performance enhancements over an engine using other cooling designs. The AVH concept could also be more easily implemented into a current gas turbine design than a more complex machining design such as shaped holes.

- Less coolant being extracted from the compressor would translate to a lessor specific work needed by the turbine.

- It is hypothesized that the decrease in cooling flow would lead to reduced aerodynamic losses in the turbine thus increasing the turbine's isentropic efficiency.

- Durability concerns on the blades could be lessened by the increased structural stability of the blades, since they would operate at lower temperatures. The AVH could require less material to be taken from the blades to achieve the same coolant coverage.

- Conversely, the engine could instead be operated at higher turbine inlet temperatures thus increasing the cycle efficiency and power output of the engine. (Important for military engines to have the highest power output and thrust to maintain air superiority.)

The blowing ratio is not a parameter that is selectable. The blowing ratio of a film cooling hole at a given location on a blade or vane is dependent on the pressure drops through the passages leading from the compressor to the hole itself. An AVH geometry would be most useful in regions where the blowing ratio is high. Additional research would be needed before implementing an AVH concept. Confirmation of the decrease in 
the aerodynamic losses in the turbine would be necessary. Design considerations would also be necessary to determine the amount that the turbine inlet temperature could be increased or the extent that the durability could increases. Either would provide an enhancement to the engine. 


\subsection{FutURE AND CONCURRENT WORK}

An experimental wind tunnel has been designed to non-dimensionally simulate the aerothermal environment experienced by the components in the first stage of a gas turbine engine. The initial focus of the research planned for this new laboratory is to evaluate and compare the performance of the AVH to validate the results from the present CFD study. The effect of increasing turbulence levels as well as various other parameters such as cooling blowing ratio, freestream Reynolds number, cooling hole spacing and approaching boundary layer height. These parameters cover the broad range of engine geometric and aerodynamic conditions and will all be capable of being simulated. The facility will be suitable for the testing of cooling on flat-plates, leading edge models (for showerhead cooling) and combustor liner and contoured endwall cooling. The facility will be used to evaluate novel cooling geometries and to validate the research in the current study that has been performed using CFD models. The primary data acquisition system will employ a transient infrared (IR) thermography technique as described in [42] and summarized in section 2.3. Particle image velocimetry (PIV) may also be employed to verify the flow field and jet lift-off. The cooling holes for flat-plate testing will be scaled to approximately ten times that of actual gas turbine dimensions to help clearly show how the cooling is effected by each cooling hole design.

Future CFD will also include LES and additional unsteady RANS cases. Experimental work in the wind tunnel may employ 2-D PIV to compare cross sectional slices and near hole interaction to cross sectional slices that can be easily extracted from the CFD computational domain. A flow visualization technique that was developed by Sarginson et al. [49] may be of interest for qualitative comparison of the experimental 
coolant jets to the numerical results. This technique uses a low aerodynamic drag mesh coated in thermochromic liquid crystals that is put into the flow downstream of the film cooling holes. Modification to this technique for use coupled with an IR camera could provide useful insight into the coolant jets interaction for relatively low cost. This technique may also provide some experimental insight into the jet lift-off and AVH interaction that could easily be compared to CFD data from this study and future studies.

The author would also suggest further optimization of the side hole locations and size requirements in future studies. The spacing of the holes should also be reevaluated to obtain the most efficient use of the cooling flow. Future work in the hole spacing and sizing could be accomplished through simple RANS models and completed in a much shorter period of time than would have been possible 10 years ago with modern computing technology. A single RANS case could be completed in less than 24 hours with an adequate mesh. Studies such as this would be valuable in further optimizing the anti-vortex hole concept. Experimental work in the newly built wind tunnel could be used to verify the results from any CFD studies. 


\title{
APPENDICES
}

\section{ApPendix A: Summer Heat Transfer Conference Paper (2013)}

\begin{abstract}
A PARAMETRIC NUMERICAL STUDY OF THE EFFECTS OF FREESTREAM TURBULENCE INTENSITY AND LENGTH SCALE ON ANTI-VORTEX FILM COOLING DESIGN AT HIGH BLOWING RATIO
\end{abstract}

Timothy W. Repko and Andrew C. Nix

Department of Mechanical and Aerospace Engineering

West Virginia University

Morgantown, WV, 26505

andrew.nix@mail.wvu.edu

\author{
James D. Heidmann \\ Turbomachinery and Heat Transfer Branch \\ NASA Glenn Research Center \\ Cleveland, Ohio 44135 \\ heidmann@nasa.gov
}

\section{ABSTRACT}

An advanced, high-effectiveness film-cooling design, the anti-vortex hole (AVH) has been investigated by several research groups and shown to mitigate or counter the vorticity generated by conventional holes and increase film effectiveness at high blowing ratios and low freestream turbulence levels. $[1,2]$ The effects of increased turbulence on the AVH geometry were previously investigated and presented by researchers at West Virginia University (WVU), in collaboration with NASA, in a preliminary CFD study [3] on the film effectiveness and net heat flux reduction (NHFR) at high blowing ratio and elevated freestream turbulence levels for the adjacent $\mathrm{AVH}$ The current paper presents the results of an extended numerical parametric study, which attempts to separate the effects of turbulence intensity and length-scale on film cooling effectiveness of the AVH. In the extended study, higher freestream turbulence intensity and larger scale cases were investigated with turbulence intensities of 5, 10 and $20 \%$ and length scales based on cooling hole diameter of $\Lambda_{\mathrm{x}} / \mathrm{d}_{\mathrm{m}}=1,3$ and 6 . Increasing turbulence intensity was shown to increase the centerline, span-averaged and area-averaged adiabatic film cooling effectiveness. Larger turbulent length scales were shown to have little to no effect on the centerline, spanaveraged and area-averaged adiabatic film-cooling effectiveness at lower turbulence levels, but slightly increased effect at the highest turbulence levels investigated.

\section{INTRODUCTION}

Both the thermal efficiency and power output of a gas turbine increase as the turbine inlet temperatures increases. This increase in temperature creates a multitude of durability issues for the components in the hot section of the turbine. In the range of temperatures gas turbines experience, increasing the temperature of hot section components by $10-20 \mathrm{~K}$ can effectively halve the operational life of a component [4]. Increasing the thermal efficiency or increasing the durability is a major tradeoff in gas turbine design.
Film-cooling has typically been employed as a fundamental technique in reducing the heat load on components in the hot section of turbine engines. In the past, film-cooling has consisted of angled cylindrical holes injecting the coolant into the hot mainstream flow allowing a cooler layer of gas close to the walls to protect the components and decrease their surface temperature. The blowing ratio or mass flux ratio, $\mathrm{M}$, is a significant parameter when looking at the performance of film-cooling and is defined as the ratio of the coolant mass flux to the freestream mass flux, $(\rho \mathrm{V})_{\mathrm{d}} /(\rho \mathrm{V})_{\text {in. }}$. Blowing ratios greater than approximately 1.5 are considered high and are often seen in practice with real engines. These high blowing ratios may cause the coolant jet to lift-off away from the wall and create a counter rotating vortex (CRV) pair. The coolant jet lift-off generally occurs at blowing ratios greater than 0.5 [1]. The CRV pulls hot gases from the freestream and entrains them near to the wall, reducing the effectiveness of the cooling film. Haven et al. [5] produced a diagram depicting the CRV pair and hot gas entrainment shown in Figure 1. The vorticity generated stems from shear interaction between the hot mainstream flow and the coolant jet as well as a secondary interaction with the solid wall.

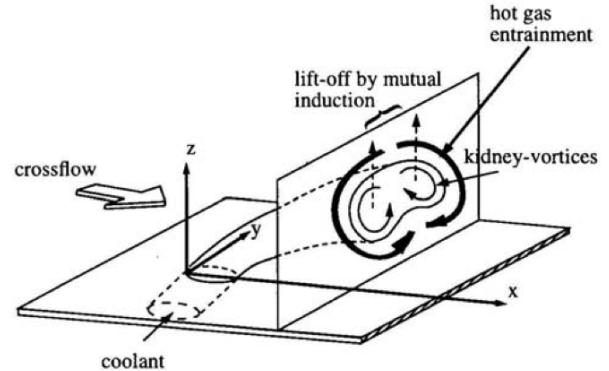

Figure 1. Illustration Of Counter Rotating Vortex [5] 
Near to the injection hole the coolant has enough momentum to keep its shape and act as a solid cylinder with the hot mainstream gases flowing around it as is the case for a cylinder in a cross flow.

The coolant that is supplied for film-cooling is extracted from high-pressure stages of the compressor. This bleed air comes at a penalty to the overall cycle performance as it decreases the mass flow through the combustor and turbine inlet, and requires a larger specific work to be done by the turbine to power the compressor. Thus, the use of the extracted cooling flow should be as efficient as possible. Numerous studies in the open literature were aimed at efficiently using the cooling flow and many novel film-cooling shapes have been proposed. Many of these novel film-cooling shapes have promising performance but are not practical in application with modern machining and casting techniques.

Under real operating conditions of the engine, the air bled off from the compressor is at a sufficiently high pressure that jet lift-off may be present and the CRV may develop. In addition to adversely affecting the cooling effectiveness, the CRV may bring particulate from the mainstream close to the surface where they can be deposited. The particulate in the mainstream flow is a product from modern integrated gasification, combined cycle (IGCC) turbines as a product of the high-hydrogen content coal synthesis gas or in modern military aircraft engines where fine siliceous debris (sand) is ingested with the intake air in the core and mainstream flows in desert operations. Deposition of this particulate material (coal ash in IGCC turbines and sand, also known as calciummagnesium-alumino-silicate or CMAS in aero engines) can cause degradation of TBCs and increase the surface roughness of turbine components leading to thermal failure of the components and further degrading the cooling performance.

Many studies have been performed in an attempt to combat the jet lift-off behavior at high blowing ratios. One of the most commonly used techniques that has had success at combating the jet lift-off is the use of shaped film-cooling holes. Bunker [6] provides a review of shaped film-cooling technology from its inception to 2005 concluding that the target for shaped filmcooling holes is to "expand the exit area in the plane of the surface of the injection jet by a factor of 2-3 times that of the round jet without separation." Expanding the area of the cooling hole decreases the momentum of the cooling jet, thus promoting an attached film.

Rigby and Heidmann [7] proposed placing a vortex generator that protrudes into the flow, downstream of a filmcooling hole to counter the CRV. While this vortex generator showed viable results in improving effectiveness, it is limited by the practicality in machining. Introducing more surfaces, which need to be cooled, could also prove problematic.

Ely et al. [8] presented the concept of using "sister holes" that are separate from and adjacent to the main cooling hole, a similar geometry to that of the adjacent AVH. The concept behind "sister holes" is to place smaller diameter holes adjacent to the main hole that are optimized to counter the CRV produced from the main hole. The exits of these sister holes are in a similar location to the exits of the anti-vortex holes in relation to the main hole. The difference between the two geometries is that the sister holes are fed directly from the inner blade plenum, whereas the AVH geometry has one main hole connected to the plenum feeding coolant to both of the side anti-vortex holes as well as the main hole. Ely et al. [8] showed that the sister holes offered a cooling advantage over the cylindrical holes across their domain which extended 30 main cooling hole diameters $\left(\mathrm{x} / \mathrm{d}_{\mathrm{m}}\right)$ downstream.

Heidmann et al. [1,2] developed the anti-vortex concept, which could possibly, through optimization, reduce or cancel the vorticity of the CRV pair, and not just lessen its effect as in shaped holes. Differing from the sister holes concept, the AVH has its secondary holes intersect the main hole allowing one inlet to feed the coolant to all of the holes as discussed earlier. A beneficial effect of this design is to slightly diffuse the coolant flow and allow it to stay attached for higher plenum pressures (blowing ratios). It is intended that the side holes interact with one another when in a row to produce a strong upwash, which must be balanced by a net downwash in the main hole jet centerline plane. The current study is concerned with investigation of the effectiveness of the AVH in the presence of elevated levels of turbulence.

Due to the fact that the mechanism for the effectiveness of the AVH is through interaction of vortical structures from the main film-cooling hole and the side cooling holes and the potential CRV, the effect that high-intensity, large-scale combustor exit freestream turbulence will have on the capability of the AVH to effectively cool airfoil surfaces is of concern. The hot gas flow leaving the combustor is not well characterized but is known to be highly turbulent. This highly turbulent flow increases the heat transfer to hot section components, especially in the first stage of a gas turbine, and can lead to thermal failure of the components. Previous studies by Van Fossen and Bunker [9] have shown that turbulence intensities can be as high as $20 \%-30 \%$ in a simulated engine environment which measured the intensity and scale of turbulence downstream of a GE90 combustor segment with cold, pressurized flow. Studies by Wang et al. [10] and Barringer et al. [11] have modeled the combustor exit turbulence in the same intensity range with length scale to blade chord length $\left(\Lambda_{\mathrm{x}} / \mathrm{c}\right)$ in the range of 0.11-0.43. Van Fossen and Bunker [5] and Nix et al. [12] showed that a realistic length scale to blade chord parameter $\left(\Lambda_{x} / \mathrm{c}\right)$ is on the order of 0.3 . This length scale normalized by the film cooling hole diameter $\left(\Lambda_{\mathrm{x}} / \mathrm{d}_{\mathrm{m}}\right)$ would be approximately $10-15$ in modern aircraft engines. The current study focuses on length scales based on the film-cooling hole diameter $\left(\Lambda_{\mathrm{x}} / \mathrm{d}_{\mathrm{m}}\right)$ between 1 and 6 , due to limitations in the maximum length scale of turbulence to be generated in the experimental validation facility currently being fabricated.

The characteristics of the turbulent flow exiting the combustor are not easily characterized and can vary widely 
depending on combustor geometry and operational conditions. Past studies, more numerous than can be covered here, have been performed to investigate the effect that elevated levels of freestream turbulence has on film-cooling hole geometries. Bons et al. [13] found that high freestream turbulence can decrease film-cooling effectiveness for baseline cylindrical cooling holes along the hole centerline but increase the effectiveness along the midline between holes. Saumweber et al. [14] showed that the effect of turbulence on shaped film cooling holes is detrimental at all blowing ratios, yet cylindrical cooling holes experience slight gains in effectiveness at high blowing ratios. Saumweber et al. [15] reiterated the findings from [14] for the cylindrical case and found that fan-shaped cooling holes also have their performance degraded by increasing the freestream turbulence.

The current study will focus on understanding the effect that the turbulent structures will have on the AVH interaction with the CRV and the resultant cooling of the wall. This understanding will be limited by using the Reynolds-Averaged Navier-Stokes RANS model and the unsteady turbulent effect will be better understood in future studies with the use of large eddy simulations, unsteady RANS, and experimental validation, including adiabatic effectiveness and Laser Doppler Velocimetry (LDV) measurements. Hunley et al. [3] conducted a preliminary study on the effects of turbulence on the filmcooling effectiveness for the optimized AVH design. Baseline cases at low turbulence levels of 5\% intensity and length scale based on cooling hole diameter of $\Lambda_{\mathrm{x}} / \mathrm{d}_{\mathrm{m}}=1$ with a nominal blowing ratio of 2 and density ratios of 1 and 2 were compared to previous results at low turbulence levels in studies by Heidmann et al. [1,2]. In the preliminary study, three turbulence conditions were studied; $5 \%$ turbulence with a $\Lambda_{\mathrm{x}} / \mathrm{d}_{\mathrm{m}}$ of $1,10 \%$ turbulence with $\Lambda_{\mathrm{x}} / \mathrm{d}_{\mathrm{m}}$ of 1 , and $10 \%$ turbulence with a $\Lambda_{\mathrm{x}} / \mathrm{d}_{\mathrm{m}}$ of 3. The current study looks to expand on the previous work to nine total cases with turbulence intensities of $5 \%, 10 \%$, and $20 \%$ as well as length scales of $\Lambda_{\mathrm{x}} / \mathrm{d}_{\mathrm{m}}$ of 1,3 , and 6 . The desired results from the current study are to investigate elevated turbulence and to attempt to separate the effects of intensity and length scale on AVH film-cooling effectiveness.

\section{TEST GEOMETRY AND COMPUTATIONAL SETUP AVH Geometry}

The AVH has been studied by Heidmann and other research collaborators in $[1,2,3,16]$ in both experimental and numerical formats. Heidmann and Ekkad [1] developed the concept of the AVH with some preliminary designs and numerical simulations. Dhungel et al. [16] looked at 6 different geometrical configurations of the AVH to determine an optimal location of the side holes in reference to the main hole using a low speed wind tunnel with IR imaging. Heidmann [2] continued the work with a numerical simulation of two of the optimized configurations determined in [16]. It was found that the location of the exits of the side cooling holes should be slightly downstream of the exit of the main film-cooling hole and should intersect the main cooling hole near the plenum.
Figure 2 shows the geometrical parameters in the optimization study of Dhungel [16] and the values describing the geometry studied herein are given in Table 1 .

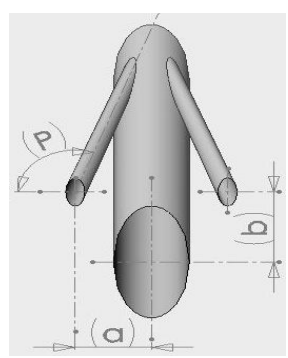

Top View

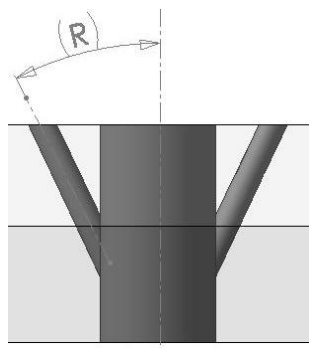

Front View

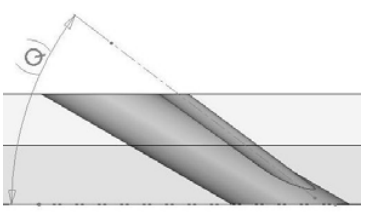

Side View

Figure 2. Generic Orthographic Projections Of The AVH [16]

Table 1. Geometric Parameters For Optimized AVH Case [1]

\begin{tabular}{|l|l|}
\hline$d_{s} / d_{m}$ & 0.5 \\
\hline$a / d_{m}$ & 1.12 \\
\hline$b / d_{m}$ & -0.44 \\
\hline$P$ & 105.67 \\
\hline$Q$ & 26.41 \\
\hline$R$ & 27.91 \\
\hline
\end{tabular}

The optimized AVH geometry modifies the basic cylindrical film-cooling hole by adding two additional holes stemming from the main cooling hole. Unlike other filmcooling hole designs such as shaped holes, a key characteristic of the AVH geometry is that the holes are simple angled cylinders to allow for simplistic manufacturing techniques. Six variations of the AVH were considered in the parametric study by Dhungel et al. [16]. The geometry that was shown to be the most structurally feasible and have the best performance at the widest range of blowing ratios, momentum ratios, and density ratios is the adjacent AVH described in Figure 2 and Table 1. Details of the computational setup of this geometry, with a description of the modeled engine conditions are presented in the following section.

\section{Computational Setup}

A multi-block structured computational grid was produced for the AVH geometry using commercially available software, GridPro $^{\mathrm{TM}}$. This computational grid contained 2.5 million structured hexahedral cells. The density of the grid was increased in regions near to solid walls where viscous effects 
would dominate using a $\mathrm{y}^{+}$value of less than 1.0 at all near wall locations. Normal to the walls, a stretching ratio of 1.2 was used. In comparison to the previous work by Hunley et al. [3], this new grid contains an order of magnitude more cells and is more refined in the viscous regions of the flow, which has a strong influence on the computational accuracy compared to the previous work that presented preliminary results.

The current work is setup in the same fashion to the preliminary study with an increase in the resolution to approximately double the resolution in each coordinate direction resulting in a mesh with 8 times as many cells, but with an emphasis in the viscous clustering region. While the total grid resolution was increased one order of magnitude, the first grid point off of the wall was refined two orders of magnitude as compared to the previous study by Hunley et al. [3]. Figure 3 shows a view of the multi-block grid produced in GridPro $^{\mathrm{TM}}$.

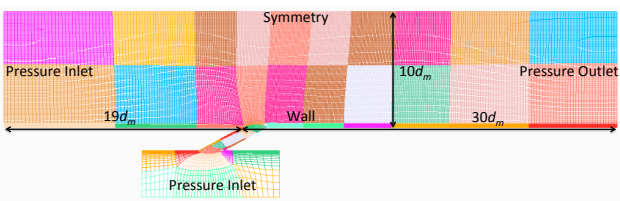

Figure 3. Grid And Setup Of Computational Domain (Modified From [2])

Figure 4 highlights the quality of the grid near the hole intersection with the cooled surface of interest. For this computational setup only half of the domain was modeled for the AVH geometry and a symmetry plane was used through the hole centerline. This can be done in this study since the computation method is using a steady RANS solver and no flow can cross through the symmetry plane. Heidmann [1] pointed out that any unsteady RANS or LES (Large Eddy Simulations) in the future would need to model the full domain and apply periodic boundary conditions. The freestream inlet is modeled $19 d_{m}$ upstream and the freestream outlet is modeled for $30 \mathrm{~d}_{\mathrm{m}}$ downstream. The top symmetry boundary condition was modeled $10 \mathrm{~d}_{\mathrm{m}}$ above the flat plate. The full RANS equations were solved using a commercial CFD code, STAR$\mathrm{CCM}+$. Consistent with previous research by $[1,2,3]$ the $\mathrm{k}-\omega$ turbulence model was used. Research by Harrison and Bogard [17] showed that the standard k- $\omega$ was the best predictor of span-averaged film effectiveness when compared to other RANS turbulence models under the conditions of their study.

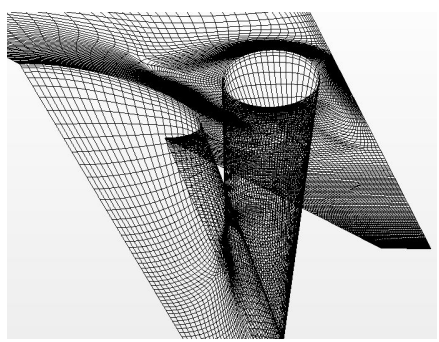

Figure 4. Mesh Resolution Near To AVH

Air was used as the fluid in the free stream and in the plenum. The viscosity of air was found through a 0.7 power law [18]. Air was considered to be an ideal gas and specific heat $\left(c_{p}\right)$ was taken to be a constant. Thermal conductivity $(k)$ was found using Sutherland's law with a reference value of 0.02414 $\mathrm{W} / \mathrm{m}-\mathrm{K}$. At the inlet to the control volume a stagnation temperature and pressure boundary condition were prescribed along with a turbulence intensity and length scale based on the case being examined. Each case in this study required different turbulence intensity and length scale in order to separate the effect of intensity and length scale on the adiabatic film-cooling effectiveness for the AVH. Turbulence intensities of 5\%,10\% and $20 \%$ were examined with length scales of 1,3 , and 6 times the main cooling hole diameter $\left(\mathrm{d}_{\mathrm{m}}\right)$, totaling nine cases. Downstream, the exit static pressure was set to 0.97 times the inlet total pressure to produce a nominal Mach number of 0.2 in the free stream. The plenum total pressure was $1-2 \%$ above the freestream value to produce a nominal blowing ratio (based on mass flux) of 2 . The plenum total temperature to freestream inlet total temperature ratio was set to be 0.5 , which is representative of modern engine conditions (range of 0.4-0.5) and is the same condition modeled by Heidmann et al. [1] and Hunley et al. [3]. This yields a density ratio of approximately 2 for all cases. The average freestream inlet conditions along with the plenum conditions were used to calculate the Reynolds number and blowing ratio. Keeping with previous research by Heidmann et al. [1,2] and Dhungel et al. [16] the Reynolds number based on the main film-cooling hole diameter and freestream fluid property and velocity conditions was 11,300.

The numerical simulations were performed on a computer benchmarked at 250 GigaFLOPS taking approximately 200 hours to complete all cases. Convergence was determined using the same criteria as [1]. Convergence was achieved for each case when all of the residuals were reduced by 3 orders of magnitude and there was no observable change in the surface temperature prediction downstream of the holes for 1000 iterations. This was accomplished using area-averaged monitors of the surface temperature as well as discrete monitors downstream of the cooling holes at the surface as well as $3 \mathrm{~d}_{\mathrm{m}}$ off of the surface. 
A summary of each of the 9 test cases for the current study is shown in Table 2 . This extends the preliminary study from 3 cases to 9 cases including a larger length scale based on main film cooling hole diameter of 6 and a higher turbulence intensity of $20 \%$ than previously tested.

Table 2. Test Matrix

\begin{tabular}{|c|c|c|}
\hline Case Number & Turbulence Intensity & Length Scale $\left(\Lambda_{\mathrm{x}} / \mathrm{d}_{\mathrm{m}}\right)$ \\
\hline 1 & 5 & 1 \\
\hline 2 & 10 & 1 \\
\hline 3 & 20 & 1 \\
\hline 4 & 5 & 3 \\
\hline 5 & 10 & 3 \\
\hline 6 & 20 & 3 \\
\hline 7 & 5 & 6 \\
\hline 8 & 10 & 6 \\
\hline 9 & 20 & 6 \\
\hline
\end{tabular}

RESULTS

Film-cooling (adiabatic) effectiveness $\left(\eta_{a w}\right)$ for a low Mach number flow is well known as:

$$
\eta_{a w}=\frac{T_{i n}-T_{a w}}{T_{i n}-T_{c}}
$$

The temperature at the pressure inlet of the computational domain as shown in Figure 3 is given as $T_{i n}$ and the coolant temperature is the plenum condition as given by $T_{c}$. Results presented herein include contours of adiabatic effectiveness, the effectiveness along the main hole centerline and the spanwise average effectiveness and their variation downstream, and the area averaged effectiveness. Also included are cross sectional temperature contours of discrete planes downstream of the cooling hole to try and better understand the physics of the mainstream interaction with the coolant.

These results extend upon a preliminary study by Hunley et al. [3]. In the preliminary study limited computational power may have affected the reported results. Yavuzkurt et al. [19] reported that there could be fairly radical difference $(>20 \%)$ in the results for a film-cooling geometry depending on the mesh resolution and type of mesh. In this case the viscous clustering near the walls may have led to an over prediction of the filmcooling effectiveness in the previous study although trends relating to turbulence level and length scale remained the same, as discussed herein. Another consideration in the difference in the results between the current and preliminary study may be the variation in implementation of the turbulence models by the different CFD codes by FLUENT and STAR-CCM+. All nine cases in the current study were run in Fluent, with slight variation, but not as significant as compared to the original study. The results of this study were compared to the preliminary study [3], experimental work by Dhungel et al. [15], and a further computational study by Heidmann et al. $[1,2]$. Current results match all of the previous work in magnitude but have variations in the flow physics. There is a longer reattachment period for the main cooling jet at low turbulence levels that was not predicted in the previous work.
This can be seen in Figure 5 in the following section and is most readily apparent for the lowest turbulence intensity and smallest length scale.

\section{Contour Plots of Effectiveness}

Contour plots of the adiabatic effectiveness allow the centerline and area-averaged effectivenes values to be put into a perspective so that one might be able to qualitatively understand the phyics of the flow for a given set of conditions and geometry. Figure 5 shows contour plots of the adiabatic effectiveness for the entire test surface of interest for various turbulence intensities and length scales. For the low turbulence cases, the lateral spreading of the coolant is not as pronounced as is the case for the higher levels of turbulence. With increasing turbulence it can clearly be seen that there is a distinct increase in lateral spreading with the most effective case being the case with the largest length scale as well as the highest turbulence intensity. For higher turbulence intensities the lateral spreading of the coolant occurs much further upstream (closer to the cooling holes), providing better coverage and improved effectiveness (span and area averaged, as dicussed later). This is in agreement with the trends in numerous past studies available in open literature involving freestream turbulence and cylindrical film-cooling for high blowing ratios $[12,13]$. While the side holes would cover more area than a conventional straight (center hole), there is still a region of low effectiveness between the main and side holes which decreases with higher Tu. This hot streak is shortened with increasing turbulence intensity, as shown in Figure 5. It is not trivial to discern the effect of length scale $\left(\Lambda_{\mathrm{x}} / \mathrm{d}_{\mathrm{m}}\right)$ by looking at the contour plots alone. The effect of length scale will be examined more closely with the centerline and spanaveraged effectiveness.

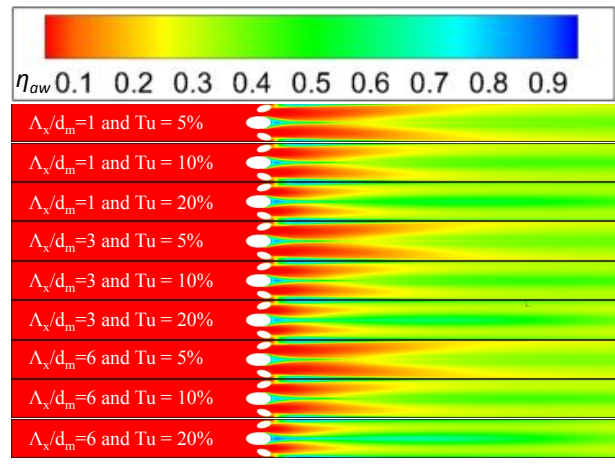

Figure 5. Contours Of Adiabatic Film-Cooling Effectiveness 


\section{Centerline Effectiveness}

Centerline effectiveness results are useful in illustrating potential cooling jet lift-off. At any streamwise point, the maximum cooling effectiveness is generally along the hole centerline and can be determined by comparing centerline effectiveness plots. Centerline effectiveness plots are shown in Figure 6 for the variation in effectiveness at fixed length scale $\left(\Lambda_{\mathrm{x}} / \mathrm{d}_{\mathrm{m}}\right)$ and varying turbulence intensity and Figure 7 for the variation in effectiveness at fixed turbulence intensity and varying length scale for each of the 9 cases. An attempt was made to present the data in the clearest manner, allowing low turbulence intensities, $5 \%$, to be the lightest color, moderate intensities, $10 \%$, to be a intermediate color, and high turbulence intensities, $20 \%$, to be the darkest color. Length scales based on main cooling hole diameter of 1,3 , and 6 are colored as blue, green, and red, respectively. It can be seen for all cases that there is no significant prediction of jet lift-off, or at least whatever lift-off there is lasts for a very short streamwise distance before re-attaching.

The centerline data starts immediately downstream of the exit of the main film-cooling hole at streamwise location, $x / \mathrm{d}_{\mathrm{m}}$, of 2.0. At this point the centerline film-cooling effectiveness is at its peak. From the maximum it steadily declines to a local minima near an $\mathrm{x} / \mathrm{d}_{\mathrm{m}}$ of 10 (with the exception of a slight increase at $\mathrm{x} / \mathrm{d}_{\mathrm{m}}$ of approximately 3 ). This indicates that the main film-cooling jet is slightly detaching from the wall but is not considered complete jet lift-off. For the higher turbulence levels the gradient is less steep and the local minima is higher indicating that the main coolant jet remains closer to the wall and better attached. Further downstream the higher turbulence levels tend to continually decrease until the end of the domain is reached whereas the lower turbulence levels tend to rise until the end of the domain. The preliminary study found that increasing the length scale from 1 to 3 at a turbulence intensity of $10 \%$ almost uniformly decreases the centerline effectiveness at all streamwise locations. The current results found that increasing the length scale in the same manner increases the centerline effectiveness almost uniformly across the domain; but is only a diminutive increase. At large length scales and high turbulence intensities there is sufficient mixing far downstream that the effectiveness falls near or below that of the lower turbulence intensities, as evidenced in Figure 6 (a-c)

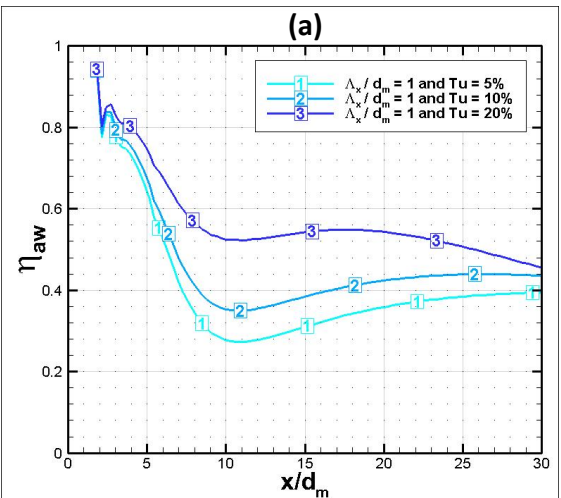

(b)

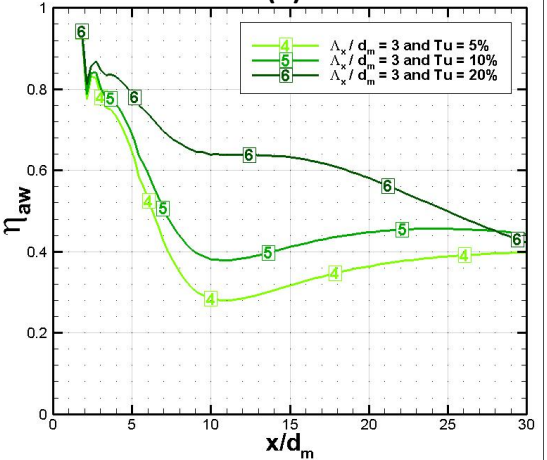

(c)

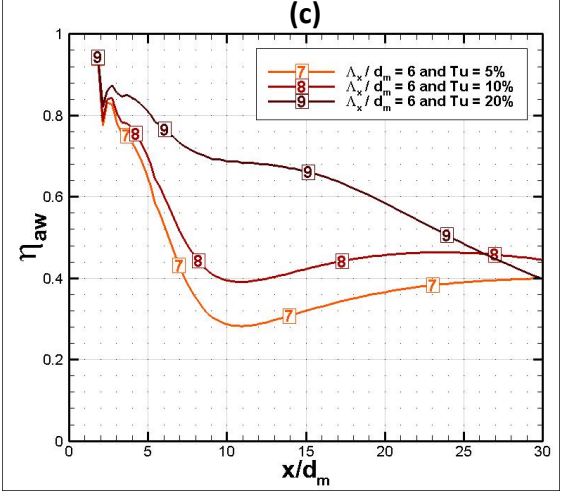

Figure 6. Centerline Effectiveness With Constant Length Scales 


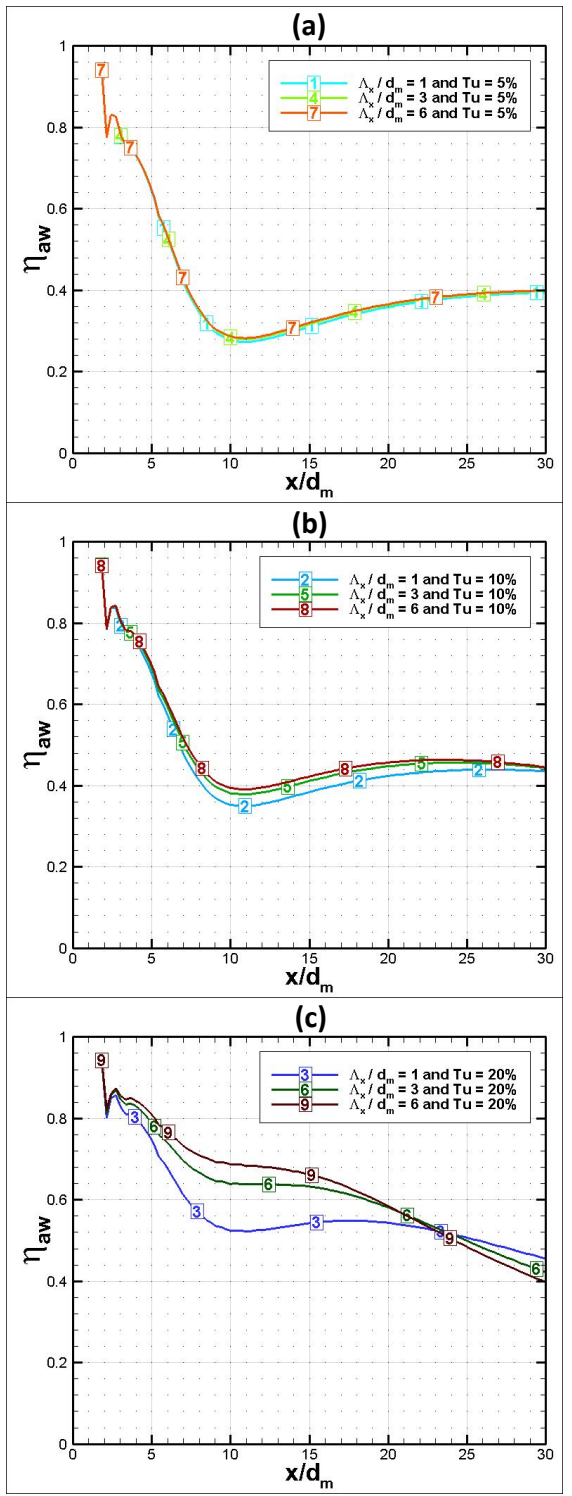

Figure 7. Centerline Effectiveness With Constant Turbulence Intensities

\section{Span Average Effectiveness}

The span-averaged film effectiveness plots were generated by averaging the effectiveness laterally over the computational domain. It may be relevant to note that the computational domain is half of what is pictured in the adiabatic effectiveness contour plots. The data was mirrored across the symmetry plane to allow for a more complete picture of the physics of the full flow while saving time by using less computational power than simulating the full span. The span-averaged film effectiveness will be analyze in an attempt to show quantitatively the effect of the increasing turbulence and length scale.

Span-averaged film effectiveness gives the best quantitative look into how well a given cooling geometry functions and, along with the qualitative results of contour plots, is the most common tool when analyzing film-cooling performance. In Figure 8 the span-averaged film-cooling effectiveness is given at constant length scales $\left(\Lambda_{\mathrm{x}} / \mathrm{d}_{\mathrm{m}}\right)$ of 1,3 and 6 in subplots (a), (b), and (c) respectively. As the turbulence intensity is increased, the span-averaged effectiveness is increased at nearly all locations in the domain, regardless of the length scale. In the case of $\Lambda_{x} / d_{m}$ of 1 , the small scale eddies do not effectively laterally mix the coolant jet as the large scale eddies do with a $\Lambda_{\mathrm{x}} / \mathrm{d}_{\mathrm{m}}$ of 6 . This can be best seen in comparing Figure 9 (a) and (c). In the current study the streamwise location of maximum cooling moves upstream with increasing turbulence intensity. At the highest turbulence intensity (Figure 9c) the effects of turbulent length scale is apparent as it moves the point of maximum effectiveness upstream. This effect is not evident at the lower turbulence levels. For case 9, with the highest turbulence intensity and length scale, the point of maximum span-averaged cooling is near an $\mathrm{x} / \mathrm{d}_{\mathrm{m}}$ of 15 . For the lowest turbulence and length scale (case 1) it appears that the point of maximum cooling is downstream of the domain $\left(x / d_{m}>30\right)$.

\section{Area Averaged Effectiveness}

The area-averaging of the effectiveness paints a broad picture of the film-cooling effectiveness at all locations of the domain downstream of the cooling hole geometry. A limitation of the area-averaged effectiveness is that hot streaks and hot spots may not have a large effect on the reported value of the area-averaged effectiveness but may be sufficiently large enough to cause localized thermal failure of components. Table 3 shows that there is a significant increase in the area averaged effectiveness with an increase in turbulence intensity. It also shows that there is a slight but noticeable increase in the areaaveraged effectiveness with increasing length scale. As a comparison to the preliminary data, there is an over prediction of effectiveness which may be a result of the difference in CFD code, implementation of the turbulence model, differences in grid spacing, or a combination of the three. 


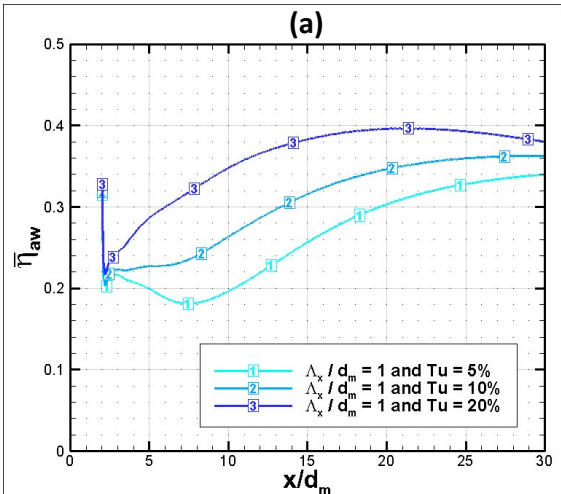

(b)

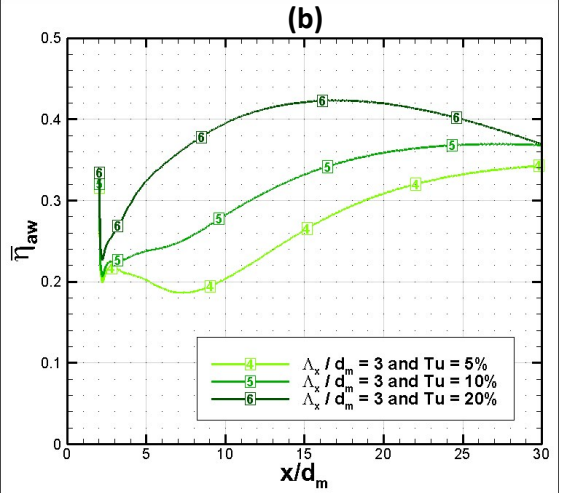

(c)

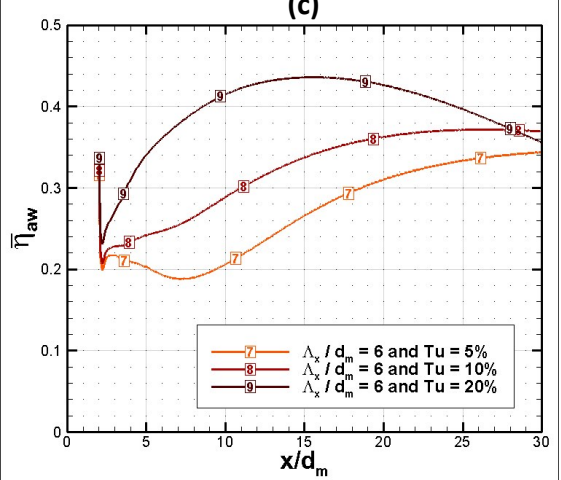

Figure 8. Span-Averaged Film Effectiveness With Constant Length Scales

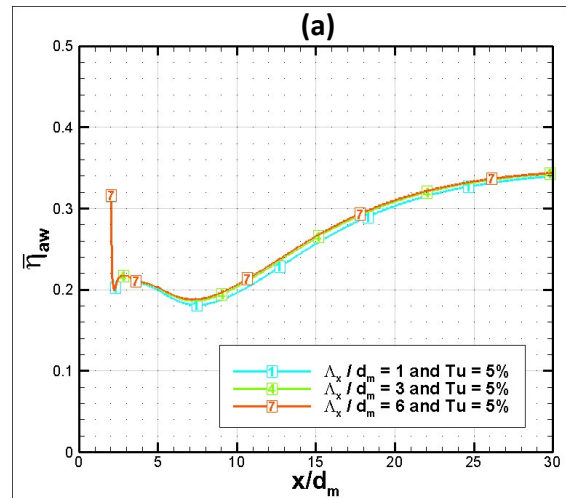

(b)

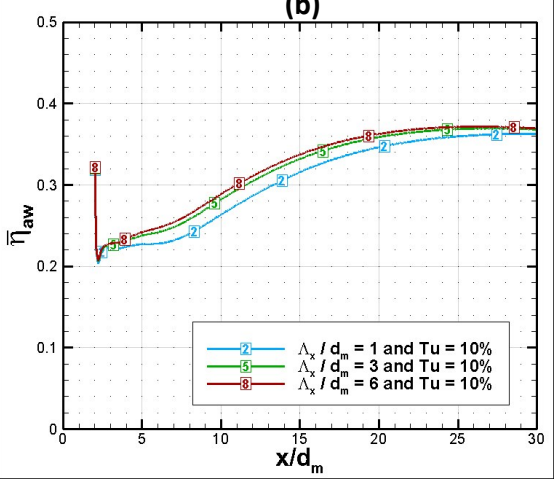

(c)

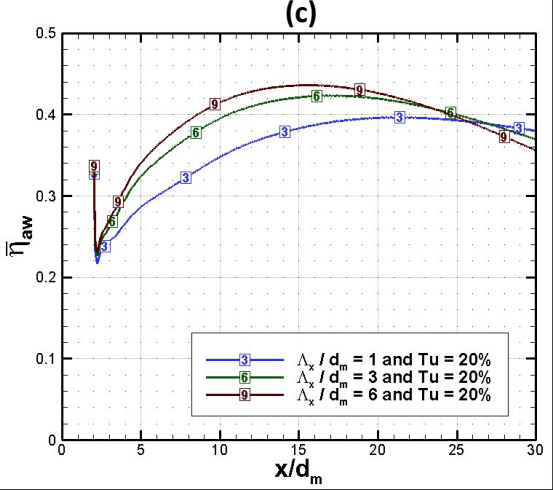

Figure 9. Span-Averaged Film Effectiveness With Constant Turbulence Levels 
Table 3. Summary Of Area-Averaged Effectiveness

\begin{tabular}{|l|l|c|}
\hline \multicolumn{1}{|c|}{ Case } & \multicolumn{2}{|c|}{ Area-Averaged Effectiveness } \\
\cline { 2 - 3 } & \multicolumn{1}{|c|}{ Current } & Previous \\
\hline $1 . \Lambda_{\mathrm{x}} / \mathrm{d}_{\mathrm{m}}=1$ and $\mathrm{Tu}=5 \%$ & 0.2657 & $0.3411[2]$ \\
\hline $2 . \Lambda_{\mathrm{x}} / \mathrm{d}_{\mathrm{m}}=1$ and $\mathrm{Tu}=10 \%$ & 0.3079 & $0.3703[2]$ \\
\hline $3 . \Lambda_{\mathrm{x}} / \mathrm{d}_{\mathrm{m}}=1$ and $\mathrm{Tu}=20 \%$ & 0.3602 & \\
\hline $4 . \Lambda_{\mathrm{x}} / \mathrm{d}_{\mathrm{m}}=3$ and $\mathrm{Tu}=5 \%$ & 0.2706 & \\
\hline $5 . \Lambda_{\mathrm{x}} / \mathrm{d}_{\mathrm{m}}=3$ and $\mathrm{Tu}=10 \%$ & 0.3200 & $0.3786[2]$ \\
\hline $6 . \Lambda_{\mathrm{x}} / \mathrm{d}_{\mathrm{m}}=3$ and $\mathrm{Tu}=20 \%$ & 0.3848 & \\
\hline $7 . \Lambda_{\mathrm{x}} / \mathrm{d}_{\mathrm{m}}=6$ and $\mathrm{Tu}=5 \%$ & 0.2722 & \\
\hline $8 . \Lambda_{\mathrm{x}} / \mathrm{d}_{\mathrm{m}}=6$ and $\mathrm{Tu}=10 \%$ & 0.3241 & \\
\hline $9 . \Lambda_{\mathrm{x}} / \mathrm{d}_{\mathrm{m}}=6$ and $\mathrm{Tu}=20 \%$ & 0.3930 \\
\hline
\end{tabular}

\section{Streamwise Total Temperature Contours}

To further understand the physics of the coolant jet interaction with the mainstream flow it is helpful to look at discrete cross sectional planes downstream of the cooling hole. Figure 10 shows dimensionless temperature contours at 4 different locations downstream of the AVH for case 9 with the largest length scale and highest turbulence intensity. This case has the highest area-averaged effectiveness and the highest peak in the span-averaged effectiveness. A dimensionless temperature $(\theta)$ is employed and is defined in a similar manner to the film-cooling effectiveness:

$$
\theta=\frac{T_{i n}-T_{f i l m}}{T_{i n}-T_{c}}
$$

In the definition of the dimensionless temperature, $T_{\text {film }}$, is a local temperature in the flow affected by the film cooling and not a bulk temperature. In the film effectiveness contours in Figure 5 and the dimensionless temperature contour in Figure 10 it can be seen that just downstream of the AVH there is a slight detachment zone where there is little mixing of the coolant from the side holes and the main hole. As the flow progresses downstream the coolant from the side holes mixes with the coolant from the main hole and begins to flatten out and cool the wall across the entire domain. The CRV seems to be reduced as the centerline cooling flow does not fully detach.

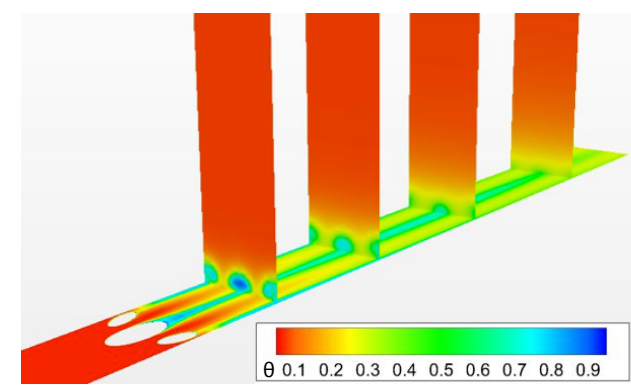

Figure 10. Dimensionless Temperature Contours At Discrete Planes Downstream Of AVH For Case 9

\section{FUTURE WORK AND EXPERIMENTAL VALIDATION}

Currently, an experimental wind tunnel is being designed to non-dimensionally simulate the aerothermal environment experienced by the components in the first stage of a gas turbine engine. The initial focus of the research of this new laboratory will be to evaluate and compare the performance of the AVH to validate the results from this study. The effect of increasing turbulence levels as well as various other parameters such as cooling blowing ratio, freestream Reynolds number, and cooling hole spacing, which cover the broad range of engine geometric and aerodynamic conditions, will be capable of being investigated. The facility will be capable of testing cooling on flat-plates, leading edge models (for showerhead cooling) and combustor liner and contoured endwall cooling. The lab will be used to evaluate novel cooling geometries and to validate the research in the current study being performed using CFD models. The primary data acquisition system will employ infrared (IR) thermography, although thermochromic liquid crystals (TLC) and pressure sensitive paint (PSP) may be used in the future if needed. LDV will also be employed to verify the flow field and jet lift-off. The cooling holes for flatplate testing will be scaled to approximately ten times that of actual gas turbine dimensions to help clearly show how the cooling is effected by each cooling hole design.

Future work with CFD will also include determination of heat transfer coefficients, such that the effectiveness data can be combined with the heat transfer coefficients, which will increase with increasing turbulence intensity, to determine the net heat flux reduction. Future CFD will also include LES and unsteady RANS. Experimental work in the wind tunnel may employ 2-D LDV to compare cross sectional slices and near hole interaction to cross sectional slices that can be easily "taken" from the computational domain. Sarginson et al. [19] developed a flow visualization technique using a low aerodynamic drag mesh coated in thermochromic liquid crystals that is put into the flow downstream of the film-cooling holes. This technique may provide some experimental insight into the jet lift-off and AVH interaction that could easily be compared to CFD data from this study and future studies. All of these numerical and experimental techniques will be employed to provide a more detailed, experimental-numerical validation data set, which will provide details of the flow physics, and flow structure interactions.

\section{CONCLUSIONS}

In the preliminary study it was found that, at high blowing ratios, and density ratios, higher freestream turbulence levels increase the film cooling effectiveness of the AVH geometry. Although there are disparities in the data compared to the previous study, the trends and magnitudes remain the same at the same blowing ratio and density ratio. High freestream turbulence does not appear to have any negative effects on the film cooling effectiveness of the AVH geometry at high blowing ratio and density ratio. The AVH geometry has been 
shown to have a substantial advantage when compared to the baseline cylindrical case from the previous study and work by Heidmann et al [1,2], Hunley et al. [3] and Dhungel et al. [15].

The elevated level of freestream turbulence increases the span-averaged, centerline, and area-averaged film cooling effectiveness for the AVH geometry. The increase in effectiveness coverage extends $30 \mathrm{~d}_{\mathrm{m}}$ downstream of the beginning of the main film-cooling hole in the majority of the cases. It is interesting to note that at large length scales and high turbulence intensities there is sufficient mixing far downstream that the effectiveness falls below that of the lower turbulence intensities. Two main conclusions can be drawn from the current study:

- It has been shown that the turbulence in the hot gases exiting the combustor can aid in the film cooling for the AVH geometry at realistic blowing ratios. However, as noted earlier, the effect of the increased convective heat transfer coefficients must be considered.

- Length scale was shown to have little to no effect at the low turbulence level and a small, but noticeable effect at high turbulence intensity. This effect may become more pronounced with non-dimensional length scales $\left(\Lambda_{\mathrm{x}} / \mathrm{d}_{\mathrm{m}}\right)$ closer to engine representative values.

As discussed, future work will investigate the NHFR and more details of the flow physics through numerical and experimental analysis.

\section{ACKNOWLEDGMENTS}

The authors would like to acknowledge the support of the Department of Energy, Office of Science, Experimental Program to Stimulate Competitive Research (EPSCoR) under grant/contract number DE-FG02-09ER46615, monitored by Dr. Tim Fitzsimmons of the DOE Office of Science/EPSCoR.

\section{NOMENCLATURE}

blade chord length

$\mathrm{d} \quad$ film cooling hole diameter

DR density ratio $\rho_{\mathrm{c}} / \rho_{\text {in }}$

$\mathrm{k} \quad$ turbulent kinetic energy

M blowing ratio or mass flux ratio $(\rho \mathrm{V})_{\mathrm{c}} /(\rho \mathrm{V})_{\text {in }}$

Re Reynolds number

T temperature

V velocity

$\mathrm{x} \quad$ streamwise direction from the hole leading edge

y normal direction from the flat plate

$\mathrm{y}^{+} \quad$ dimensionless wall unit

$\mathrm{z} \quad$ spanwise direction from the hole centerline

\section{Greek}

$\eta \quad$ adiabatic effectiveness

$\theta \quad$ dimensionless temperature

$\Lambda_{\mathrm{x}} \quad$ streamwise integral length scale

$\begin{array}{ll}\rho & \begin{array}{l}\text { density } \\ \text { specific dissipation } \varepsilon / \kappa\end{array} \\ \begin{array}{l}\text { Subscripts } \\ \text { aw }\end{array} & \begin{array}{l}\text { adiabatic wall conditions } \\ \text { coolant conditions } \\ \text { freestream inlet conditions } \\ \text { in }\end{array} \\ \text { film } & \begin{array}{l}\text { focal temperatures in freestream affected by } \\ \text { main hole }\end{array} \\ \mathrm{m} & \text { side hole or AVH } \\ \text { s } & \\ \text { Abbreviations } & \\ \text { AVH } & \text { anti-vortex hole } \\ \text { CFD } & \text { computational fluid dynamics } \\ \text { CRV } & \text { counter rotating vortex } \\ \text { CMAS } & \text { calcium-magnesium-alumino-silicate } \\ \text { FLOPS } & \text { floating operations per second } \\ \text { IGCC } & \text { integrated gasification combined cycle } \\ \text { IR } & \text { infrared } \\ \text { LES } & \text { large eddy simulations } \\ \text { LDV } & \text { laser Doppler velocimetry } \\ \text { NHFR } & \text { net heat flux reduction } \\ \text { PSP } & \text { pressure sensitive paint } \\ \text { RANS } & \text { Reynolds averaged Navier-Stokes } \\ \text { TBC } & \text { thermal barrier coating } \\ \text { TLC } & \text { thermochromic liquid crystals }\end{array}$

REFERENCES

[1] Heidmann, J.D., and Ekkad, S., 2007, "A Novel AntiVortex Turbine Film-Cooling Hole Concept," ASME Paper GT2007-27528

[2] Heidmann, J.D., 2008, "A Numerical Study of AntiVortex Film Cooling Designs at High Blowing Ratio," ASME Paper GT2008-50845.

[3] Hunley, B.K., Nix, A.C., Heidmann, J.D., 2010, “A Preliminary Numerical Study on the Effects of High Freestream Turbulence on Anti-Vortex Film Cooling Design at High Blowing Ratio," ASME Paper GT201022077.

[4] Cumpsty, N., 2003, Jet Propulsion, p.56, Cambridge University Press, NY, NY.

[5] Haven, B.A., Yamagata, D.K., Kurosaka, M., Yamawaki, S., and Maya, T., 1997, "Anti-Kidney Pair of Vortices in Shaped Holes and their Influence on Film Cooling Effectiveness," ASME Paper 97-GT-45.

[6] Bunker, R.S, 2005, "A Review of Shaped Hole Turbine Film-Cooling Technology," Journal of Heat Transfer, Vol. 127, pp. 441-453

[7] Rigby, D.L., and Heidmann, J.D., 2008, "Improved Film Cooling Effectiveness By Placing a Vortex Generator Downstream of Each Hole," ASME Paper GT200851361 
[8] Ely, M.J. and Jubran, B. A., 2008, "A Numerical Study on Increasing Film Cooling Effectiveness Through the Use of Sister Holes," ASME Paper GT2008-50366.

[9] Van Fossen, G.J., and Bunker, R.S., 2002, “Augmentation of Stagnation Region Heat Transfer Due to Turbulence from an Advanced Dual-Annular Combustor," $A S M E$ Paper GT-2002-30184.

[10] Wang, H.P., Goldstein, J., and Olson, R.J., 1998, "Effect of High Freestream Turbulence with Large Scale on Blade Heat/Mass Transfer", ASME 98-GT-107.

[11] Barringer, M.D., Richard, O.T., Walter, J.P., Stitzel, S.M., and Thole, K.A., 2001, "Flowfield Simulations of a Gas Turbine Combustor," ASME 2001-GT-0170.

[12] Nix, A.C., Smith, A.C., Diller, T.E., Ng, W.F., and Thole, K.A., 2002, "High Intensity, Large Length-Scale Freestream Turbulence Generation in a Transonic Turbine Cascade," ASME Paper GT-2002-30523.

[13] Bons, J.P., MacArthur, C.D., Rivir, R.B., 1996, "The Effect of High Freestream Turbulence on Film Cooling Effectiveness," Journal of Turbomachinery, Vol. 118, pp. 814-825.

[14] Saumweber, C., Schulz, A., and Wittig, S., 2002, "FreeStream Turbulence Effects on Film Cooling with Shaped Holes," ASME Paper GT2002-30170.

[15] Saumweber, C., and Schultz, A., 2012, " Free-Stream Effects on the Cooling Performance of Cylindrical and Fan-Shaped Cooling Holes," ASME Journal of Turbomachinery, Vol. 134, No. 6, November 2012, 061007.

[16] Dhungel, S., Phillips, A., Ekkad, S.V., and Heidmann, J.D., 2007, "Experimental Investigation of a Novel AntiVortex Film Cooling Hole Design," ASME Paper GT2007-27419.

[17] Harrison, K.L., and Bogard, D.G, 2008, "Comparison of RANS Turbulence Models for Prediction of Film Cooling Performance," ASME Paper GT2008-51423.

[18] Schlicting, H., 1979, Boundary Layer Theory, $7^{\text {th }}$ edition, p. 312-313, McGraw-Hill, NY, NY.

[19] Yavuzkurt, and S., Habte, M., 2008, "Effect of Computational Grid on Performance of Two-Equation Models of Turbulence for Film Cooling Applications," ASME Paper GT2008-50153.

[20] Sargison, J.E., Guo, S.M., Oldfield, M.L.G., Lock, G.D., and Rawlinson, A.J., 2002, "Flow Visualization of a Converging Slot-Hole Film-Cooling Geometry," ASME Paper GT-2002-30177. 


\section{ApPEndix B: Draft of ASME Journal Of TuRbomachinery Publication}

\section{A PARAMETRIC NUMERICAL STUDY OF THE EFFECTS OF FREESTREAM TURBULENCE INTENSITY AND LENGTH SCALE ON ANTI-VORTEX FILM COOLING DESIGN AT HIGH BLOWING RATIO}

Timothy W. Repko and Andrew C. Nix

Department of Mechanical and Aerospace Engineering

West Virginia University

Morgantown, WV, 26505

andrew.nix@mail.wvu.edu

\section{Abstract}

An advanced, high-effectiveness film cooling design, the anti-vortex hole (AVH) has been investigated by several research groups and shown to mitigate or counter the vorticity generated by conventional holes and increase film effectiveness at high blowing ratios and low freestream turbulence levels. [1-3] The effects of increased turbulence on an AVH geometry were previously investigated in a preliminary steady CFD study by Hunley et al. [4] on the film effectiveness and net heat flux reduction (NHFR) at high blowing ratio. The current paper presents the results of an extended numerical parametric study, which attempts to separate the effects of turbulence intensity and length-scale on film cooling performance of the AVH concept. In the extended study, higher freestream turbulence intensity and larger scale cases were investigated with turbulence intensities of 5, 10 and $20 \%$ and length scales based on cooling hole diameter of $\Lambda_{\mathrm{x}} / \mathrm{d}_{\mathrm{m}}=1,3$ and 6 . Increasing turbulence intensity was shown to increase the centerline, span-averaged and areaaveraged adiabatic film cooling effectiveness and NHFR. Larger turbulent length scales were shown to have little to no effect on the centerline, span-averaged and area-averaged adiabatic film cooling effectiveness and NHFR at lower turbulence levels, but moderate effect at the highest turbulence levels investigated. Heat transfer results were able to reiterate the findings from adiabatic cases from previous work [5] Turbulent length scales were shown to have little effect, thus three Unsteady RANS simulations were carried out at the three turbulence intensities at constant length scale, $\Lambda_{\mathrm{x}} / \mathrm{d}_{\mathrm{m}}=1$. Averaged URANS data shows a decreased prediction in film cooling effectiveness when compared to the RANS data

\section{Introduction}

Both the thermal efficiency and power output of a gas turbine increase as the turbine inlet temperature increases. This increase in temperature creates a multitude of durability issues for the components in the hot section of the turbine. In the range of temperatures gas turbines experience, increasing the temperature of hot section components by $10-20 \mathrm{~K}$ can effectively halve the operational life of a component [6]. Increasing the thermal efficiency or increasing the durability is a major tradeoff in gas turbine design.
James D. Heidmann

Turbomachinery and Heat Transfer Branch

NASA Glenn Research Center

Cleveland, Ohio 44135

heidmann@nasa.gov

Film cooling has typically been employed as a fundamental technique in reducing the heat load on components in the hot section of turbine engines. Film cooling consists of angled cylindrical holes injecting the coolant into the hot mainstream flow allowing a cooler layer of gas close to the walls to protect the components and decrease their surface temperature. The blowing ratio or mass flux ratio, $\mathrm{M}$, is a significant parameter when looking at the performance of film cooling and is defined as the ratio of the coolant mass flux to the freestream mass flux, $(\rho \mathrm{V})_{\mathrm{c}} /(\rho \mathrm{V})_{\text {in }}$. Blowing ratios greater than approximately 1.5 are considered high and are often seen in practice with real engines. These high blowing ratios may cause the coolant jet to lift-off away from the wall and create a counter rotating vortex (CRV) pair. The coolant jet lift-off generally occurs at blowing ratios greater than 0.5 . [3] The CRV pulls hot gases from the freestream and entrains them near to the wall, reducing the effectiveness of the cooling film. Haven et al. [7] produced a diagram depicting the CRV pair and hot gas entrainment shown in Figure 1. The vorticity generated stems from shear interaction between the hot mainstream flow and the coolant jet as well as a secondary interaction with the solid wall. Near to the injection hole the coolant has enough momentum to keep its shape and act similar to a solid cylinder with the hot mainstream gases flowing around it as is the case for a cylinder in a cross flow.

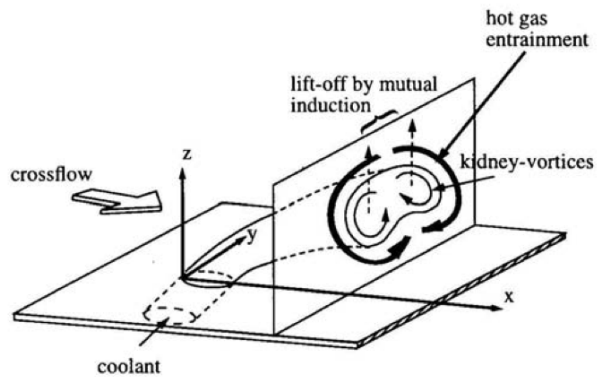

Figure 1. Illustration of Counter Rotating Vortex (CRV) [7]

The coolant that is supplied for film cooling is extracted from high-pressure stages of the compressor. This bleed air comes at a penalty to the overall cycle performance as it decreases the mass flow through the combustor and turbine 
inlet, and requires a larger specific work to be done by the turbine to power the compressor. Thus, the use of the extracted cooling flow should be as efficient as possible. Numerous studies in the open literature were aimed at efficiently using the cooling flow and many novel film cooling shapes have been proposed. Many of these novel film cooling shapes have promising performance but are not practical in application with modern machining and casting techniques.

Under real operating conditions of the engine, the air bled off from the compressor is at a sufficiently high pressure that jet lift-off may be present and the CRV may develop. In addition to adversely affecting the cooling effectiveness, the CRV may bring particulate from the mainstream close to the surface where they can be deposited. The particulate in the mainstream flow is a product from modern integrated gasification, combined cycle (IGCC) turbines as a product of the high-hydrogen content coal synthesis gas or in modern military aircraft engines where fine siliceous debris (sand) is ingested with the intake air in the core and mainstream flows in desert operations. Deposition of this particulate material (coal ash in IGCC turbines and sand, also known as calciummagnesium-alumino-silicate or CMAS in aero engines) can cause degradation of thermal barrier coatings and increase the surface roughness of turbine components leading to thermal failure of the components and further degrading the cooling performance.

Many studies have been performed in an attempt to combat the jet lift-off behavior at high blowing ratios. One of the most commonly used techniques that has had success at combating the jet lift-off is the use of shaped film cooling holes. Bunker [8] provides a review of shaped film cooling technology from its inception to 2005 concluding that the target for shaped film cooling holes is to "expand the exit area in the plane of the surface of the injection jet by a factor of 2-3 times that of the round jet without separation." Expanding the area of the cooling hole decreases the momentum of the cooling jet, thus promoting an attached film.

Rigby and Heidmann [9] proposed placing a vortex generator that protrudes into the flow, downstream of a film cooling hole to counter the CRV. While this vortex generator showed viable results in improving effectiveness, it is limited by the practicality in machining. Introducing more surfaces, which need to be cooled, could also prove problematic.

Ely et al. [10] presented the concept of using "sister holes" that are separate from and adjacent to the main cooling hole, a similar geometry to that of the adjacent AVH. The concept behind "sister holes" is to place smaller diameter holes adjacent to the main hole that are optimized to counter the CRV produced from the main hole. The exits of these sister holes are in a similar location to the exits of the antivortex holes in relation to the main hole. The difference between the two geometries is that the sister holes are fed directly from the inner blade plenum, whereas the AVH geometry has one main hole connected to the plenum feeding coolant to both of the side anti-vortex holes as well as the main hole. Ely et al. [10] showed that the sister holes offered a cooling advantage over the cylindrical holes across their domain which extended 30 main cooling hole diameters $\left(\mathrm{x} / \mathrm{d}_{\mathrm{m}}\right)$ downstream.

Heidmann et al. [1-3] developed the anti-vortex concept, which could possibly, through optimization, reduce or cancel the vorticity of the CRV pair, and not just lessen its effect as in shaped holes. Differing from the sister holes concept, the AVH has its secondary holes intersect the main hole allowing one inlet to feed the coolant to all of the holes as discussed earlier. A beneficial effect of this design is to slightly diffuse the coolant flow and allow it to stay attached for higher plenum pressures (blowing ratios). It is intended that the side holes interact with one another when in a row to produce a strong upwash, which must be balanced by a net downwash in the main hole jet centerline plane.

Concurrent to this research, LeBlanc et al. [11] continued to develop the AVH concept and changed the geometry so that the side holes were of the same diameter of the main hole. Also included in the study was a trenched AVH design that showed improvements in the trench but reduced effectiveness further downstream. This AVH design used $50 \%$ less coolant than cylindrical holes with a $30 \%-40 \%$ increase in overall averaged effectiveness.

Due to the fact that the mechanism for the effectiveness of the AVH is through interaction of vortical structures from the main film cooling hole and the side cooling holes and the potential CRV, the effect that high-intensity, large-scale combustor exit freestream turbulence will have on the capability of the AVH to effectively cool airfoil surfaces is of concern. The hot gas flow leaving the combustor is not well characterized but is known to be highly turbulent. This highly turbulent flow increases the heat transfer to hot section components, especially in the first stage of a gas turbine, and can lead to thermal failure of the components. Previous studies by Van Fossen and Bunker [12] have shown that turbulence intensities can be as high as $20 \%-30 \%$ in a simulated engine environment. This study measured the intensity and scale of turbulence downstream of a GE90 combustor segment with cold, pressurized flow. Studies by Wang et al. [13] and Barringer et al. [14] and Nix [15] showed that a realistic length scale to blade chord parameter $\left(\Lambda_{\mathrm{x}} / \mathrm{c}\right)$ is on the order of 0.3 . This length scale normalized by the film cooling hole diameter $\left(\Lambda_{\mathrm{x}} / \mathrm{d}_{\mathrm{m}}\right)$ would be approximately $10-15$ in modern aircraft engines. The current study focuses on length scales based on the film cooling hole diameter $\left(\Lambda_{\mathrm{x}} / \mathrm{d}_{\mathrm{m}}\right)$ between 1 and 6 , due to limitations in the maximum length scale of turbulence to be generated in the experimental validation facility currently being fabricated. The characteristics of the turbulent flow exiting the combustor are not easily characterized and can vary widely depending on combustor geometry and operational conditions. Past studies, more numerous than can be covered here, have been performed to investigate the effect that elevated levels of freestream turbulence has on film cooling hole geometries. Bons et al. [16] found that high freestream turbulence can decrease film 
cooling effectiveness for baseline cylindrical cooling holes along the hole centerline but increase the effectiveness along the midline between holes. Saumweber et al. [17] showed that the effect of turbulence on shaped film cooling holes is detrimental at all blowing ratios, yet cylindrical cooling holes experience slight gains in effectiveness at high blowing ratios. The findings from this study were reiterated in [18] for the cylindrical case and it was found that fan-shaped cooling holes also have their performance degraded by increasing the freestream turbulence.

Hunley et al. [4] conducted a preliminary study on the effects of turbulence on film cooling effectiveness for the most feasible AVH design found by Dhungel et al. [1]. Baseline cases at low turbulence levels of 5\% intensity and length scale based on cooling hole diameter of $\Lambda_{\mathrm{x}} / \mathrm{d}_{\mathrm{m}}=1$, with a nominal blowing ratio of 2 and density ratios of 1 and 2 , were compared to previous results at low turbulence levels in studies by Heidmann et al. [2, 3]. In the preliminary study, three turbulence conditions were studied; $5 \%$ turbulence with $\Lambda_{\mathrm{x}} / \mathrm{d}_{\mathrm{m}}$ of $1,10 \%$ turbulence with $\Lambda_{\mathrm{x}} / \mathrm{d}_{\mathrm{m}}$ of 1 , and $10 \%$ turbulence with $\Lambda_{\mathrm{x}} / \mathrm{d}_{\mathrm{m}}$ of 3 . Nine steady adiabatic cases, nine steady heat transfer cases, and three unsteady cases are considered in the present work. This study is limited to RANS models of the film cooling flow, both steady and unsteady. Length scales of $\Lambda_{\mathrm{x}} / \mathrm{d}_{\mathrm{m}}$ of 1,3 , and 6 are considered in the steady RANS cases. The effect of length scale was found to be minimal in these cases. Three unsteady RANS simulations at constant length scale were conducted to further explore the effect of increasing turbulence intensity on the film cooling effectiveness of the AVH. The desired results from the current study are to investigate elevated turbulence and to attempt to separate the effects of intensity and length scale on AVH film cooling effectiveness and better understand the cooling jets interaction with one another as well as the interaction with the mainstream flow.

Test Geometry and Computational Setup

AVH Geometry

The AVH concept has been researched [1-5] in both experimental and numerical studies. Heidmann and Ekkad [3] developed the concept of the AVH with some preliminary designs and numerical simulations. Dhungel et al. [1] looked at six different geometrical configurations of the AVH to determine an optimal location of the side holes in reference to the main hole using a low speed wind tunnel with IR imaging. Heidmann [2] continued the work with a numerical simulation of two of the optimized configurations determined in [1]. It was found that the location of the exits of the side cooling holes should be slightly downstream of the exit of the main film cooling hole and should intersect the main cooling hole near the plenum. Unlike other film cooling hole designs such as shaped holes, a key characteristic of the AVH geometry is that the holes are simple angled cylinders to allow for simplistic manufacturing techniques. Figure 2 shows the geometrical parameters in the optimization study of Dhungel et al. [1] and the values describing the specific AVH geometry studied herein are given in Table 1. The main film cooling hole is angled at $30^{\circ}$.

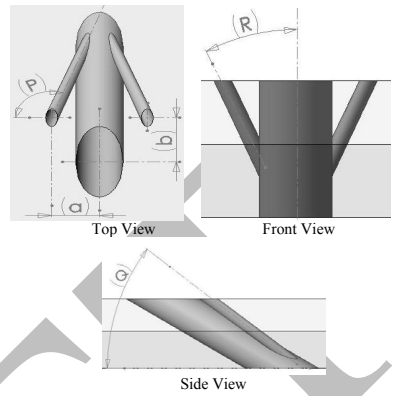

Figure 2. Generic Orthographic Projections Of The AVH [1]

Table 1. Geometric Parameters For AVH Case [2]

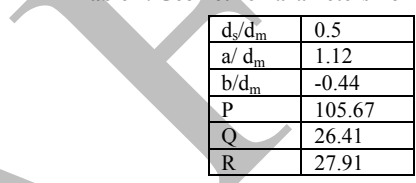

Computational Setup

A multi-block structured computational grid was produced for the AVH geometry using commercially available software, GridPro ${ }^{\mathrm{TM}}$. This computational grid contained 2.5 million structured hexahedral cells. The density of the grid was increased in regions near to solid walls where viscous effects would dominate with a $\mathrm{y}^{+}$value of much less than 1.0 at all near wall locations. Normal to the walls, a stretching ratio of 1.2 was used. In comparison to the previous work by Hunley et al. [4], this new grid contains an order of magnitude more cells and is more refined in the viscous regions of the flow, which has a strong influence on the computational accuracy compared to the previous work that presented preliminary results. The current work is conducted in the same fashion to the preliminary study with an increase in the resolution to approximately double the resolution in each coordinate direction resulting in a mesh with 8 times as many cells, but with an emphasis in the viscous clustering region. While the total grid resolution was increased one order of magnitude, the first grid point off of the wall was refined two orders of magnitude as compared to the previous study. Figure 3 shows a view of the multi-block grid produced in GridPro ${ }^{\mathrm{TM}}$.

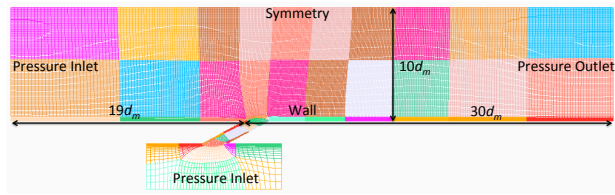

Figure 3. Grid And Setup Of Computational Domain (Modified From [2]) 
Figure 4 highlights the quality of the grid near the hole intersection with the cooled surface of interest. For this computational setup only half of the domain was modeled for the AVH geometry and a symmetry plane was used through the hole centerline. This is a valid assumption in the steady cases since a steady RANS solver is being utilized and no flow can cross through the symmetry plane. Heidmann [2] pointed out that any unsteady RANS or LES (Large Eddy Simulations) would need to model the full domain and apply periodic boundary conditions. Thus, a new grid was created for the full domain in the unsteady cases to allow for perturbations to cross the main hole centerline. A trimmed hexahedral grid containing 4.4 million cells was created in Star-CCM+.

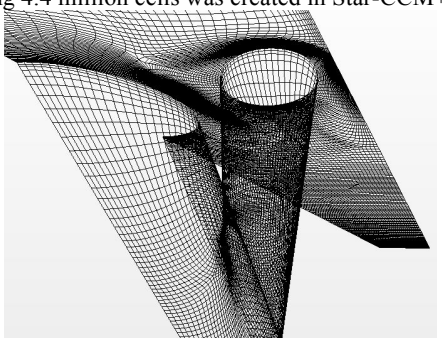

Figure 4. Mesh Resolution Near AVH Geometry (Structured)

A trimmed hexahedral mesh is predominantly a hexahedral mesh with minimal cell skewness. The bulk of the mesh is created as a hexahedral mesh and then trimmed using the input surfaces. This produces a hexahedral mesh that is nearly inline with the freestream flow direction and can produce highly accurate results in these areas. Near to the surface the cells that are trimmed are polyhedral cells. Viscous clustering was employed at the near wall locations with a $y+$ near unity. An additional level of refinement was done to increase the resolution near to the coolant jet interaction with the mainstream. This was done by enclosing a box shaped volume around the coolant jet starting as shown in Figure 5. The resulting enclosed cells were 60 percent of the base mesh size in this area.

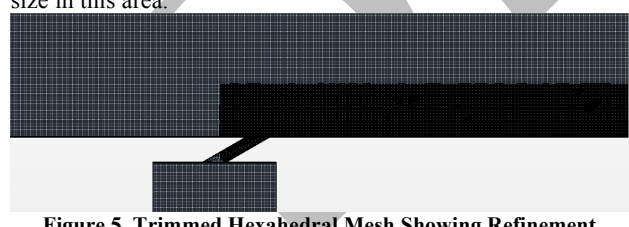

Figure 5. Trimmed Hexahedral Mesh Showing Refinement

The freestream inlet is modeled $19 \mathrm{~d}_{\mathrm{m}}$ upstream and the freestream outlet is modeled for $30 \mathrm{~d}_{\mathrm{m}}$ downstream. The top symmetry boundary condition was modeled $10 \mathrm{~d}_{\mathrm{m}}$ above the flat plate. The full RANS equations were solved using a commercial CFD code, STAR-CCM+. Consistent with previous research by [2-4] the k- $\omega$ (SST) turbulence model was used with the compressibility correction. Research by Harrison and Bogard [19] showed that the standard k- $\omega$ was the best predictor of span-averaged film effectiveness when compared to other RANS turbulence models under the conditions of their study.

Air was used as the fluid in the free stream and in the plenum. The viscosity of air was found through a 0.7 power law [20]. Air was considered to be an ideal gas and specific heat $\left(c_{p}\right)$ was taken to be a constant. Thermal conductivity $(k)$ was found using Sutherland's law with a reference value of $0.02414 \mathrm{~W} / \mathrm{m}-\mathrm{K}$. At the inlet to the control volume a stagnation temperature and pressure boundary condition were prescribed along with a turbulence intensity and length scale based on the case being examined. Each steady, adiabatic case and each heat transfer case in this study required different turbulence intensity and length scale in order to separate the effect of intensity and length scale on the adiabatic film cooling effectiveness for the AVH. Turbulence intensities of $5 \%, 10 \%$ and $20 \%$ were examined with length scales of 1,3 , and 6 times the main cooling hole diameter $\left(\mathrm{d}_{\mathrm{m}}\right)$, totaling nine adiabatic cases and nine heat transfer cases. Downstream, the exit static pressure was set to 0.97 times the inlet total pressure to produce a nominal Mach number of 0.2 in the free stream. The plenum total pressure was $1-2 \%$ above the freestream value to produce a nominal blowing ratio (based on mass flux) of 2. The plenum total temperature to freestream inlet total temperature ratio was set to be 0.5 , which is representative of modern engine conditions (range of 0.4-0.5) and is the same condition modeled by Heidmann et al. [2] and Hunley et al. [4]. This yields a density ratio of approximately 2 for all cases. The average freestream inlet conditions along with the plenum conditions were used to calculate the Reynolds number and blowing ratio. Keeping with previous research [2-4], the Reynolds number based on the main film cooling hole diameter and freestream fluid property and velocity conditions was 11,300. The nine steady adiabatic cases and three unsteady cases prescribed an adiabatic condition at the walls. A heat transfer coefficient is needed to determine the NHFR hence a specified heat flux is prescribed at the wall for the nine heat transfer cases.

The steady numerical simulations were performed on a six-core desktop computer taking 500-1000 CPU hours to complete the steady adiabatic cases. Prescribing a specified heat flux caused convergence to take up to $25-50 \%$ longer depending on the turbulence intensity and length scale. Convergence was achieved for each case when all of the residuals were reduced by 3 orders of magnitude and there was no observable change in the surface temperature prediction downstream of the holes for 1000 iterations. This was accomplished using area-averaged monitors of the surface temperature as well as discrete monitors downstream of the cooling holes at the surface as well as $3 \mathrm{~d}_{\mathrm{m}}$ off of the surface.

A summary of each of the nine test conditions for the current study is shown in Table 2. This extends the preliminary study from three cases to nine cases including a larger length scale based on main film cooling hole diameter of 6 and a higher turbulence intensity of $20 \%$ than previously tested. Heat transfer analysis is also added for all nine cases. 
Additional unsteady analysis is included for three of the nine test conditions for further investigation of the effect of turbulence intensity above the steady RANS model.

Table 2. Test Matrix

\begin{tabular}{|c|c|c|c|}
\hline $\begin{array}{c}\text { Case } \\
\text { Number }\end{array}$ & $\begin{array}{c}\text { Turbulence } \\
\text { Intensity }\end{array}$ & $\begin{array}{c}\text { Length Scale } \\
\left(\Lambda_{\mathrm{x}} / \mathrm{d}_{\mathrm{m}}\right)\end{array}$ & Analysis Method \\
\hline 1 & 5 & 1 & RANS, URANS \\
\hline 2 & 10 & 1 & RANS, URANS \\
\hline 3 & 20 & 1 & RANS, URANS \\
\hline 4 & 5 & 3 & RANS \\
\hline 5 & 10 & 3 & RANS \\
\hline 6 & 20 & 3 & RANS \\
\hline 7 & 5 & 6 & RANS \\
\hline 8 & 10 & 6 & RANS \\
\hline 9 & 20 & 6 & RANS \\
\hline
\end{tabular}

\section{Results}

Film cooling (adiabatic) effectiveness $\left(\eta_{a w}\right)$ for a low Mach number flow is well known as:

$$
\eta_{a w}=\frac{T_{i n}-T_{a w}}{T_{i n}-T_{c}}
$$

The temperature at the pressure inlet of the computational domain as shown in Figure 3 is given as $T_{\text {in }}$ and the coolant temperature is the plenum condition is given by $T_{c}$. The temperature at the wall for the adiabatic cases is $T_{a w}$ and varies spatial across the domain

Heat transfer to a film cooled blade may be defined as shown in Equation 2 [21]. Sen et al. [22] introduced the NHFR as a parameter to determine the net benefit of film cooling compared to the no film cooling case. The goal behind film cooling is to increase the NHFR by reducing the dimensionless heat transfer coefficient, $\mathrm{h}_{\mathrm{f}} / \mathrm{h}_{0}$, and increasing the adiabatic film cooling effectiveness, $\eta_{\text {aw }}$.

$$
\begin{aligned}
q_{w} & =h_{f}\left(T_{w}-T_{a w}\right) \\
N H F R & =1-\frac{h_{f}}{h_{0}}\left(1-\eta_{a w} \theta\right)
\end{aligned}
$$

A dimensionless temperature, $\theta$, is defined in Equation 4 and a value of 1.6 [3] is considered representative of engine conditions and assumes that that $h_{\mathrm{f}}$ is not a function of temperature.

$$
\theta=\frac{T_{i n}-T_{c}}{T_{i n}-T_{w}}
$$

In order to comprehensively examine the performance of the AVH, the results presented herein include analysis for the adiabatic and heat transfer cases. For the adiabatic cases the contours of adiabatic effectiveness, the effectiveness along the main hole centerline, the spanwise averaged effectiveness, and the area averaged effectiveness are examined for the steady cases. The span averaged and area averaged vales of the dimensionless heat transfer coefficient and NHFR are included for the heat transfer cases. Figures to show the development of the coolant jets and the interaction between the main coolant jet and the side coolant jets are examined for the unsteady adiabatic cases. This is accomplished through temperature isosurfaces and can help better understand the physics of the mainstream interaction with the coolant.

In the preliminary study limited computational power may have affected the reported results. Yavuzkurt et al. [23] reported that there could be fairly radical difference $(>20 \%)$ in the results for a film cooling geometry depending on the mesh resolution and type of mesh. In this case the reduced viscous clustering near the walls may have led to an over prediction of the film cooling effectiveness in the previous study, although trends relating to effects of turbulence level and length scale remained the same, as discussed herein. The results of this study were compared to the preliminary study [3], experimental work by Dhungel et al. [1], and a further computational study by Heidmann et al. [2]. Current results match all of the previous work in magnitude but have variations in the flow physics. There is a longer reattachment period for the main cooling jet at low turbulence levels that was not predicted in the previous work. This can be seen in the following section and is most readily apparent for the lowest turbulence intensity and smallest length scale.

\section{Contour Plots of Effectiveness}

Contour plots of the adiabatic effectiveness allow the centerline and area-averaged effectiveness values to be put into a perspective so that one might be able to qualitatively understand the physics of the flow for a given set of conditions and geometry. Figure 6 shows contour plots of the adiabatic effectiveness for the entire test surface of interest for various turbulence intensities and length scales. For the low turbulence cases, the lateral spreading of the coolant in the near hole region is not as pronounced as is the case for the higher levels of turbulence. With increasing turbulence it can clearly be seen that there is a distinct increase in lateral spreading with the most effective case in terms of coolant coverage being the case with the largest length scale as well as the highest turbulence intensity. For higher turbulence intensities the start of the lateral spreading of the main coolant jet occurs much further upstream (closer to the cooling holes), providing better coverage and improved effectiveness (span and area-averaged, as discussed later). This is in agreement with the trends in numerous past studies available in open literature involving freestream turbulence and cylindrical film cooling for high blowing ratios $[15,16]$. While the side holes would cover more area than a conventional straight (center hole), there is still a region of low effectiveness between the main and side holes which decreases with higher intensity. This hot streak is shortened with increasing turbulence intensity, as shown in Figure 6. It is not trivial to discern the effect of length scale $\left(\Lambda_{\mathrm{x}} / \mathrm{d}_{\mathrm{m}}\right)$ by looking at the contour plots alone. The effect of length scale will be examined more closely with the centerline and span-averaged effectiveness. 


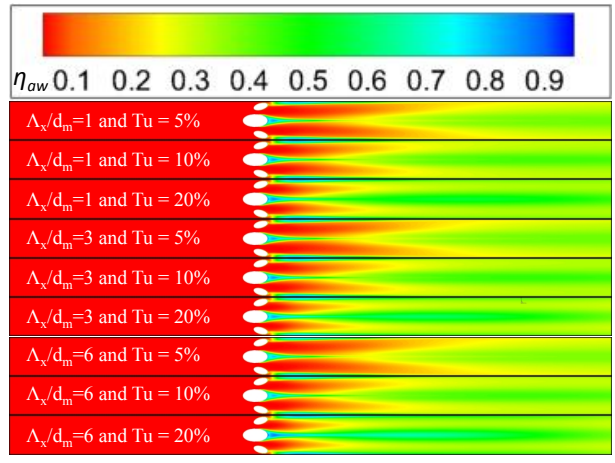

Figure 6. Contours of Adiabatic Film cooling Effectiveness

\section{Centerline Effectiveness}

Centerline effectiveness results are useful in illustrating potential cooling jet lift-off. At any streamwise point, the maximum cooling effectiveness is generally along the hole centerline and can be determined by comparing centerline effectiveness plots. Centerline effectiveness plots are shown in the (a) through (c) of Figure 7 for the variation in effectiveness at fixed length scale $\left(\Lambda_{\mathrm{x}} / \mathrm{d}_{\mathrm{m}}\right)$ and varying turbulence intensity in the (d) through (f) of Figure 7 for the variation in effectiveness at fixed turbulence intensity and varying length scale for each of the 9 cases. An attempt was made to present the data in the clearest manner, allowing low turbulence
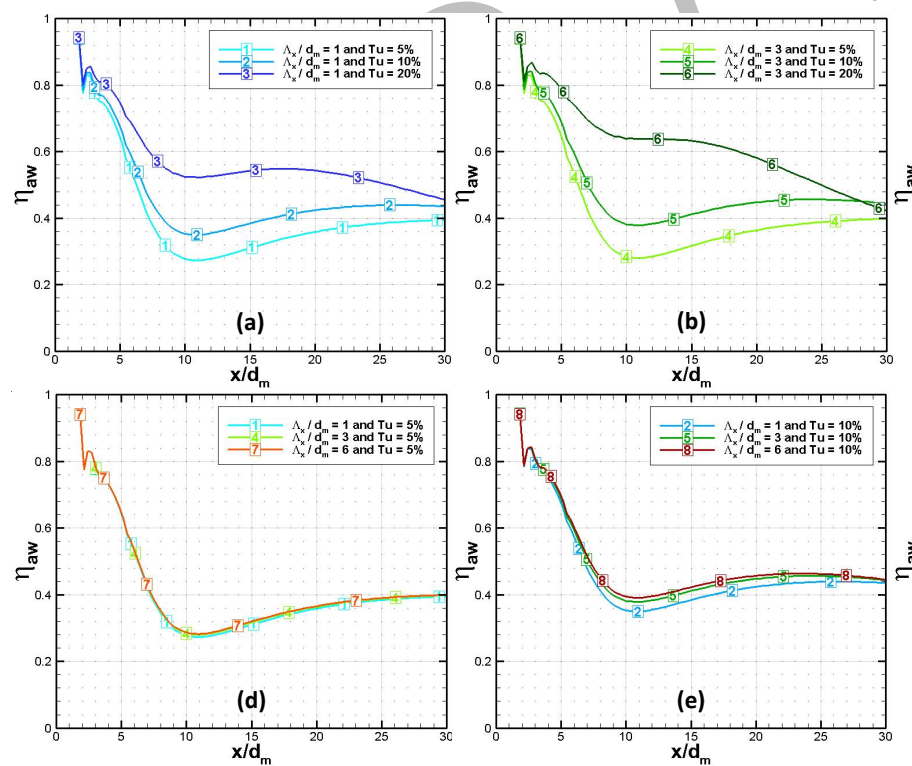

$\mathbf{x} / \mathbf{d}_{\mathbf{m}}$

Figure 7. Centerline Effectiveness At Constant Le intensities, $5 \%$, to be the lightest color, moderate intensities, $10 \%$, to be a intermediate color, and high turbulence intensities, $20 \%$, to be the darkest color. Length scales based on main cooling hole diameter of 1,3 , and 6 are colored as blue, green, and red, respectively. It can be seen for all cases that there is no significant prediction of jet lift-off, or at least whatever lift-off there is lasts for a very short streamwise distance before re-attaching. The origin for the coordinate system is at the hole leading edge.

The centerline data starts immediately downstream of the exit of the main film cooling hole at streamwise location, $\mathrm{x} / \mathrm{d}_{\mathrm{m}}$, of 2.0. At this point the centerline film cooling effectiveness is at its peak. From the maximum it steadily declines to a local minima near an $x / d_{m}$ of 10 (with the exception of a slight increase at $\mathrm{x} / \mathrm{d}_{\mathrm{m}}$ of approximately 3 ). This indicates that the main film cooling jet is slightly detaching from the wall but is not considered complete jet lift-off. For the higher turbulence levels the gradient is less steep and the local minima is higher indicating that the main coolant jet remains closer to the wall and better attached. Further downstream the higher turbulence levels tend to continually decrease until the end of the domain is reached whereas the lower turbulence levels tend to rise gradually before leveling off at the end of the domain. The preliminary study found that increasing the length scale from 1 to 3 at a turbulence intensity of $10 \%$ almost uniformly decreases the centerline effectiveness at all streamwise locations. The current results found that increasing the length scale in the same manner increases the centerline effectiveness almost uniformly across the domain; but is only a diminutive increase. At large length scales and high turbulence intensities
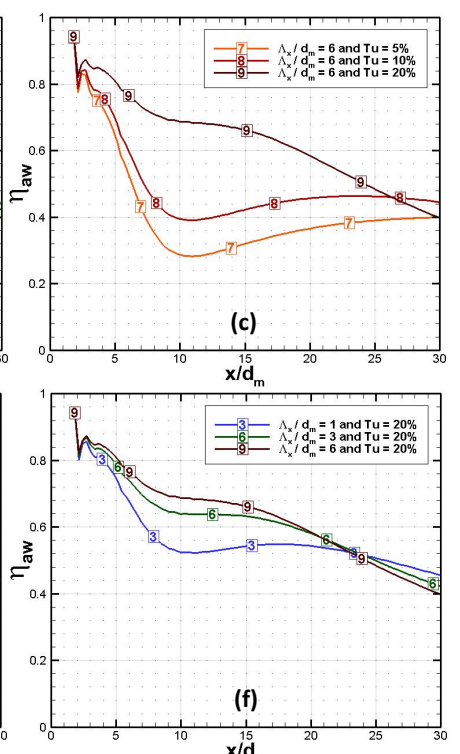
there is sufficient mixing far downstream that the effectiveness falls near or below that of the lower turbulence intensities, as evidenced in Figure 7 (a) through (c).

\section{Span-Averaged Effectiveness}

Span-averaged film effectiveness was investigated by averaging the effectiveness laterally over the computational domain and plotting vs streamwise distance, $\mathrm{x} / \mathrm{d}_{\mathrm{m}}$. It should be noted that the computational domain is half of what is pictured in the adiabatic effectiveness contour plots. The data was mirrored across the symmetry plane to allow for a more complete picture of the physics of the full flow while saving time by using less computational power than simulating the full span.

Span-averaged film effectiveness gives the best quantitative look into how well a given cooling geometry functions and, along with the qualitative results of contour plots, is the most useful tool when analyzing film cooling performance. In the subplots (a) through (c) of Figure 8 the span-averaged film cooling effectiveness is given at constant length scales $\left(\Lambda_{\mathrm{x}} / \mathrm{d}_{\mathrm{m}}\right)$ of 1,3 and 6 respectively. As the turbulence intensity is increased, the span-averaged effectiveness is increased at nearly all locations in the domain, regardless of the length scale. In the case of $\Lambda_{x} / d_{m}$ of 1 , the small scale eddies do not effectively laterally mix the coolant jet as the large scale eddies do with a $\Lambda_{\mathrm{x}} / \mathrm{d}_{\mathrm{m}}$ of 6 . This can be best seen in comparing Figure 8 (a) and (c). In the current study the streamwise location of maximum cooling moves upstream with increasing turbulence intensity at all length scales. At the highest turbulence intensity (Figure 8f) the effects of turbulent length scale is apparent as it moves the
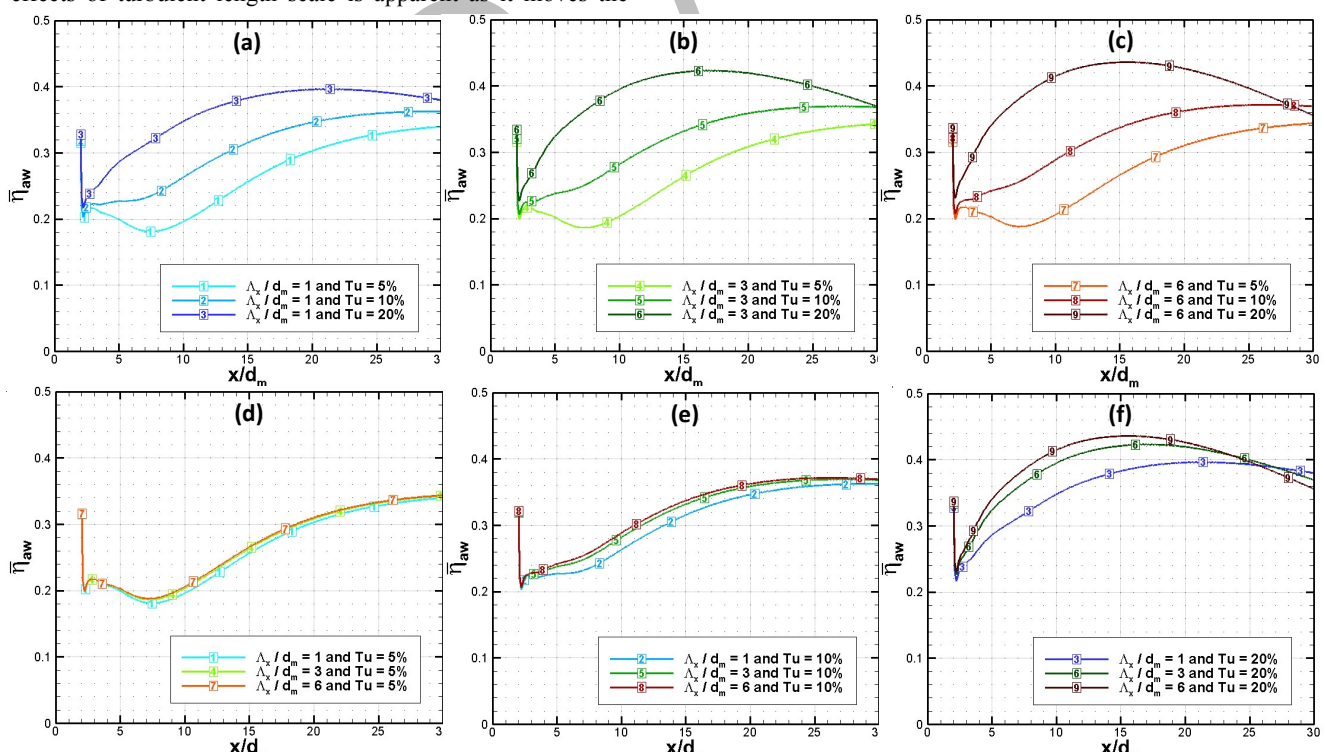

Figure 8. Span-Averaged Effectiveness At Constant Length Scale (a-c) and Turbulence Intensity (d-f)

Copyright (c) 2014 by ASME point of maximum effectiveness upstream. This effect is not evident at the lower turbulence levels. For case 9, with the maximum span-averaged cooling is near astreamwise location $\left(\mathrm{x} / \mathrm{d}_{\mathrm{m}}\right)$ of 15 . At the same turbulence intensity but at a lower of 20-22. For the lowest turbulence and length scale (case 1) it appears that the point of maximum cooling is

The area-averaging of the effectiveness paints a broad picture of the film cooling effectiveness at all locations of the domain downstream of the cooling hole geometry. A limitation of the area-averaged effectiveness is that hot streaks and hot spots may not have a large effect on the reported value of the area-averaged effectiveness but may be sufficiently large enough to cause localized thermal failure of components. Table 3 shows that there is a significant increase in the area intensity. It also shows that there is a slight but noticeable increase in the area-averaged effectiveness with increasing ength scale. As a comparison to the preliminary data, there is an over prediction of effectiveness which may be a result of the difference in CFD code, implementation of the turbulence model, differences in grid spacing, or a combination of the three. downstream of the domain $\left(\mathrm{x} / \mathrm{d}_{\mathrm{m}}>30\right)$. 
Table 3. Area-Averaged Effectiveness

\begin{tabular}{|l|c|c|}
\hline \multicolumn{1}{|c|}{ Case } & \multicolumn{2}{|c|}{ Area-Averaged Effectiveness } \\
\cline { 2 - 3 } & Current & Previous \\
\hline $1 . \Lambda_{\mathrm{x}} / \mathrm{d}_{\mathrm{m}}=1$ and $\mathrm{Tu}=5 \%$ & 0.2657 & $0.3411[4]$ \\
\hline $2 . \Lambda_{\mathrm{x}} / \mathrm{d}_{\mathrm{m}}=1$ and $\mathrm{Tu}=10 \%$ & 0.3079 & $0.3703[4]$ \\
\hline $3 . \Lambda_{\mathrm{x}} / \mathrm{d}_{\mathrm{m}}=1$ and $\mathrm{Tu}=20 \%$ & 0.3602 & \\
\hline $4 . \Lambda_{\mathrm{x}} / \mathrm{d}_{\mathrm{m}}=3$ and $\mathrm{Tu}=5 \%$ & 0.2706 & \\
\hline $5 . \Lambda_{\mathrm{x}} / \mathrm{d}_{\mathrm{m}}=3$ and $\mathrm{Tu}=10 \%$ & 0.3200 & $0.3786[4]$ \\
\hline $6 . \Lambda_{\mathrm{x}} / \mathrm{d}_{\mathrm{m}}=3$ and $\mathrm{Tu}=20 \%$ & 0.3848 & \\
\hline $7 . \Lambda_{\mathrm{x}} / \mathrm{d}_{\mathrm{m}}=6$ and $\mathrm{Tu}=5 \%$ & 0.2722 & \\
\hline $8 . \Lambda_{\mathrm{x}} / \mathrm{d}_{\mathrm{m}}=6$ and $\mathrm{Tu}=10 \%$ & 0.3241 & \\
\hline 9. $\Lambda_{\mathrm{x}} / \mathrm{d}_{\mathrm{m}}=6$ and $\mathrm{Tu}=20 \%$ & 0.3930 & \\
\hline
\end{tabular}

\section{Span-Averaged Heat Transfer}

The span averaging for the dimensionless heat transfer coefficient and NHFR was calculated in the same manner as the effectiveness. The dimensionless heat transfer was shown to reach a maximum immediately downstream of the AVH in all cases. High turbulence intensity tends to increase the peak of the maximum heat transfer coefficient downstream of the hole, but the location remains the same. This peak in heat transfer coefficient is visible in the dimensionless heat transfer coefficient plots shown in Figure 9. There is a secondary peak in the heat transfer around 4-7 hole diameters downstream that is of interest in the lower turbulence cases. This secondary peak disappears at high turbulence and large length scale cases (Case 6 and 9). The secondary peak in heat transfer occurs at or near the minimum of the NHFR in all cases as shown in Figure 10 . The location of the secondary peak was taken into
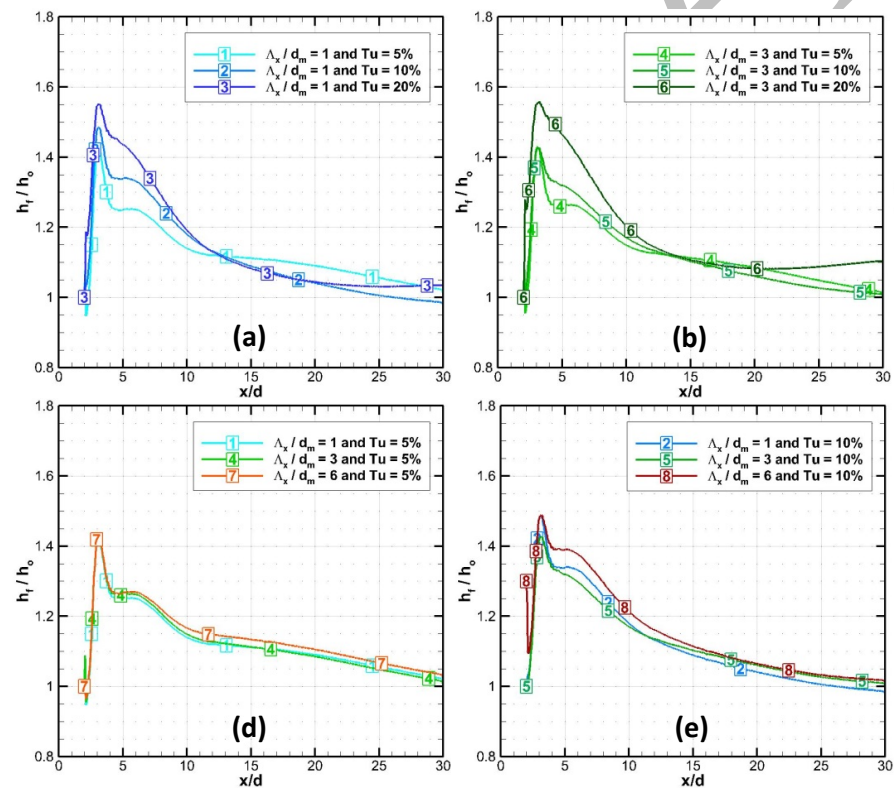

Figure 9. Span-Averaged Dimensionless Heat Transfer Coefficient account when analyzing the contours of the NHFR and it was noticed that the area between the holes could be an issue in the cooling. Trenched designs of the AVH as studied by LeBlanc et al. [11] could address this potential issue. High turbulence cases reaching a peak in the NHFR at a streamwise location of approximately $\mathrm{x} / \mathrm{d}_{\mathrm{m}}=15$ and then declines downstream of the peak. The lower turbulence cases increase throughout the domain after the local minimum a near $\mathrm{x} / \mathrm{d}_{\mathrm{m}}=4-7$. It appears that the maximum in NHFR for these lower turbulence cases is downstream of the domain as in the span-averaged effectiveness.

Overall, the increases in the heat transfer coefficient do not degrade the performance of the AVH enough to overshadow the adiabatic effectiveness. The highest turbulence intensity and length scale case has the highest NHFR with a maximum value around 15 hole diameters downstream. As in the case of the adiabatic effectiveness, turbulence intensity and length scale tends to increase the NHFR and thus the cooling performance of the AVH. Length scale is shown in the heat transfer cases to have little effect at low turbulence levels and an increasing effect as turbulence intensity is increased

\section{Area-Averaged Heat Transfer}

The area-averaged values are beneficial in the fact that one can get a generalized idea on the impact of altering the various parameters. Depicted in Table 4 , as the turbulence intensity is increased, the NHFR is noticeably increased as well. Conversely, increasing the turbulent length scale has a
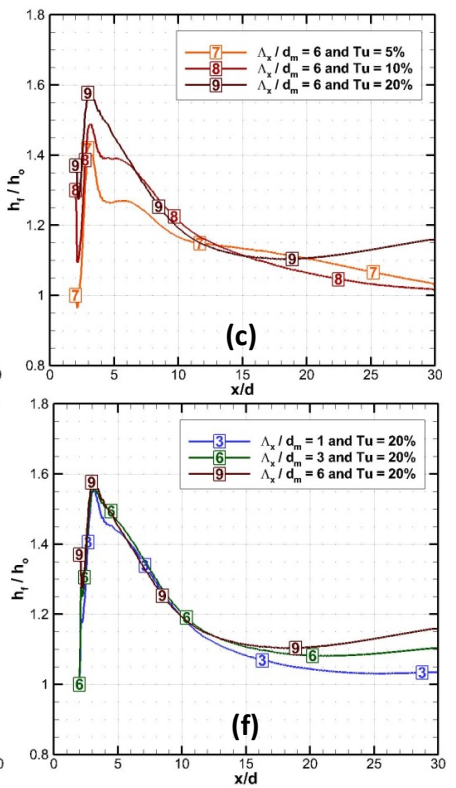

nce Intensity (d-f)

Copyright (C) 2014 by ASME 

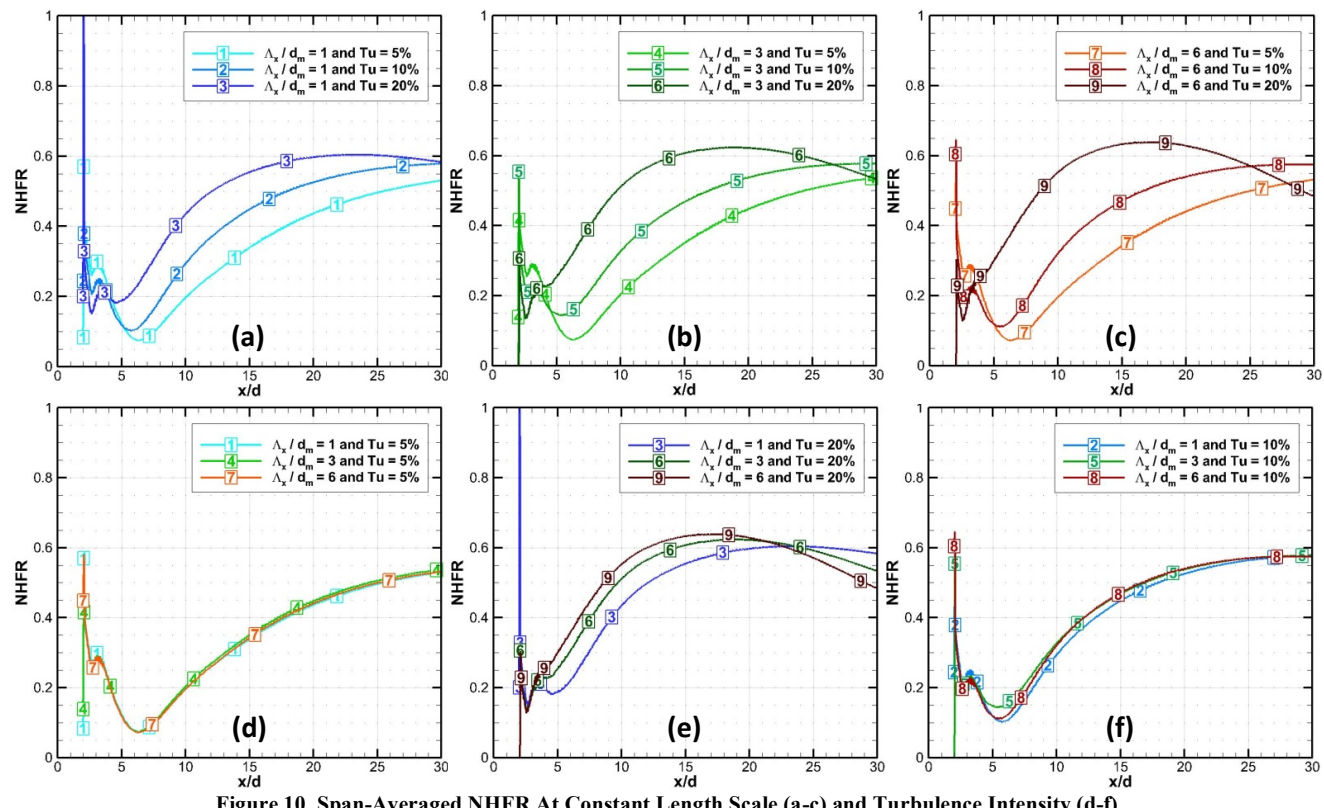

Figure 10. Span-Averaged NHFR At Constant Length Scale (a-c) and Turbulence Intensity (d-f)

much smaller effect on the NHFR at the lower length scales but does in fact have a more discernable effect in increasing NHFR at larger length scales.

When examining the dimensionless heat transfer coefficient, an increase in turbulence results in only a minor increase of the aver-averaged HTC. The length scale has limited effects on the HTC, but once again has a more discernable effect in increasing the HTC at larger length scales. It is important to reiterate that the increase in heat transfer coefficient due to film cooling being present does not over power the adiabatic effectiveness. The net benefit of the film cooling is quantified by the NHFR.

\begin{tabular}{|l|c|c|}
\multicolumn{1}{|c|}{ Case } & \multicolumn{2}{c|}{ Area-Averaged Values } \\
\cline { 2 - 3 } & $h / h_{0}$ & NHFR \\
\hline $1 . \Lambda_{\mathrm{x}} / \mathrm{d}_{\mathrm{m}}=1$ and $\mathrm{Tu}=5 \%$ & 1.123 & 0.3415 \\
\hline $2 . \Lambda_{\mathrm{x}} / \mathrm{d}_{\mathrm{m}}=1$ and $\mathrm{Tu}=10 \%$ & 1.121 & 0.4098 \\
\hline $3 . \Lambda_{\mathrm{x}} / \mathrm{d}_{\mathrm{m}}=1$ and $\mathrm{Tu}=20 \%$ & 1.147 & 0.4845 \\
\hline $4 . \Lambda_{\mathrm{x}} / \mathrm{d}_{\mathrm{m}}=3$ and $\mathrm{Tu}=5 \%$ & 1.124 & 0.3487 \\
\hline $5 . \Lambda_{\mathrm{x}} / \mathrm{d}_{\mathrm{m}}=3$ and $\mathrm{Tu}=10 \%$ & 1.125 & 0.4260 \\
\hline $6 . \Lambda_{\mathrm{x}} / \mathrm{d}_{\mathrm{m}}=3$ and $\mathrm{Tu}=20 \%$ & 1.182 & 0.5127 \\
\hline $7 . \Lambda_{\mathrm{x}} / \mathrm{d}_{\mathrm{m}}=6$ and $\mathrm{Tu}=5 \%$ & 1.141 & 0.3429 \\
\hline $8 . \Lambda_{\mathrm{x}} / \mathrm{d}_{\mathrm{m}}=6$ and $\mathrm{Tu}=10 \%$ & 1.151 & 0.4219 \\
\hline $9 . \Lambda_{\mathrm{x}} / \mathrm{d}_{\mathrm{m}}=6$ and $\mathrm{Tu}=20 \%$ & 1.198 & 0.5240 \\
\hline
\end{tabular}

\section{Comparison of RANS and URANS Simulations}

Figure 12 shows a contour plot comparing the results of unsteady effects with that of steady effects for a dimensionless length scale of one with the three aforementioned turbulence intensities. This figure shows the unsteadiness of the coolant jet in its interaction with the freestream flow. The unsteady analysis reinforces the trends of the steady work showing that increases in the turbulence intensity will lead to increases the film cooling effectiveness. When averaged over time, it should be noted that URANS predicts a higher effectiveness than the steady RANS analysis. It was shown by Voigt et al. [24] that URANS has a better prediction of the film cooling jet interaction when compared to experimental results.

Analysis of the difference between the URANS instantaneous and averaged values, as shown in Figure 11, shows a qualitative view of the unsteadiness that exists in the coolant jet. The case shown below is case $2, \Lambda_{\mathrm{x}} / \mathrm{d}_{\mathrm{m}}=1$ and $\mathrm{Tu}$ $=10 \%$. A vortex shedding exists in the wake of the cooling holes with alternating cool and hot spots being apparent in the surface temperatures. The vortex shedding is washed out as the flow convects downstream and is almost completely gone by $\mathrm{x} / \mathrm{d}=15$.

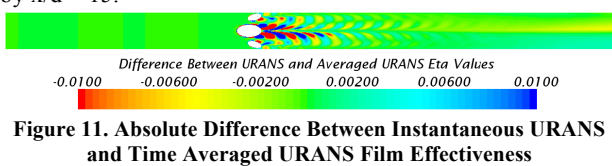
and Time Averaged URANS Film Effectiveness 


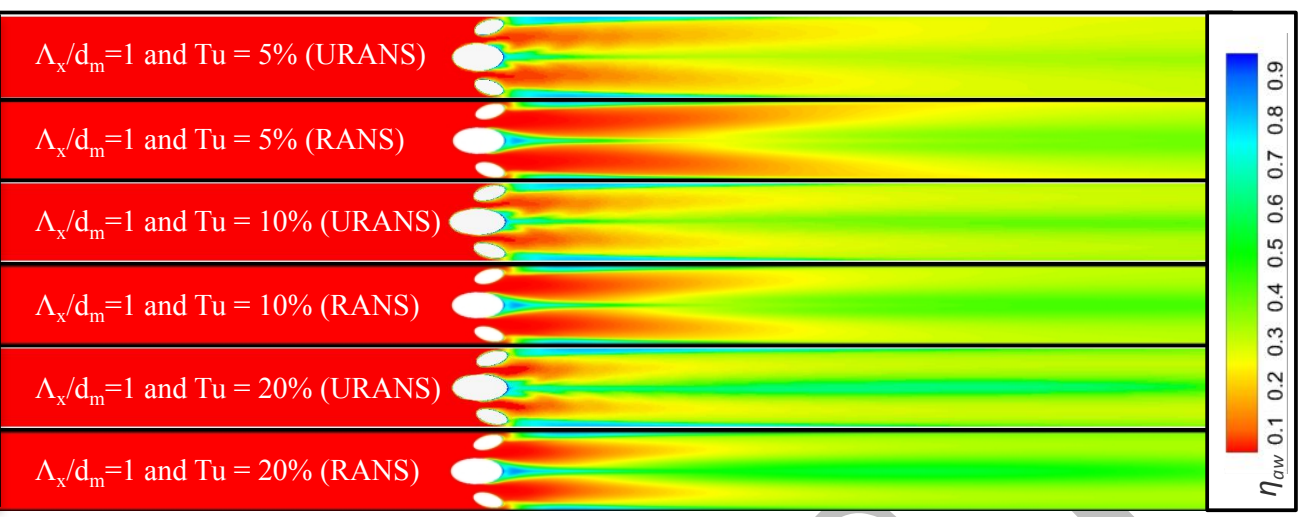

Figure 12. Contour Plots of RANS and URANS Predictions of Adiabatic Effectiveness

It is difficult to define the coolant jet and be able to visualize the interaction with the mainstream. In an attempt to qualitatively understand the mainstream coolant interaction an isosurface was created with a constant value of temperature across all three cases. This isosurface, shown in grey, represents all the points of a constant temperature in the flow and the authors feel that it gives an adequate depiction of the coolant jets.

The three URANS cases are shown in Figure 13 with the lowest turbulence cases at the top. In the lowest turbulence cases, $\mathrm{Tu}=5 \%$, the main and secondary coolants jets are able to penetrate further into the mainstream flow than the higher turbulence cases. The "ripples" from the "vortex shedding" are apparent in the temperature isosurface are also more distinct and propagate further downstream before being "washed out". At the highest turbulence intensity, $\mathrm{Tu}=20 \%$, the coolant shows the best attachment to the surface and the best overall coverage of the area downstream of the AVH.

In the film effectiveness contours in Figure 12 and the isosurface temperature plots in Figure 13 it can be seen that just downstream of the AVH there is a slight detachment zone where there is little mixing of the coolant from the side holes and the main hole. As the flow progresses downstream the coolant from the side holes mixes with the coolant from the main hole and begins to flatten out and cool the wall across the entire domain. The CRV seems to be reduced as the centerline cooling flow does not fully detach.

\section{Future Work and Experimental Validation}

An experimental wind tunnel has been designed to nondimensionally simulate the aerothermal environment experienced by the components in the first stage of a gas turbine engine. The initial focus of the research of this new laboratory will be to evaluate and compare the performance of the AVH to validate the results from this study. The effect of increasing turbulence levels as well as various other parameters such as cooling blowing ratio, freestream Reynolds number, cooling hole spacing and the approaching boundary layer thickness and profile. These parameters cover the broad range of engine geometric and aerodynamic conditions and are capable of being simulated. The primary data acquisition system will employ a transient infrared (IR) thermography technique as described in [25]. Particle image velocimetry (PIV) may also be employed to verify the flow field and jet lift-off. Future CFD will also include LES, DES, and additional unsteady RANS cases to compare to experimental results.
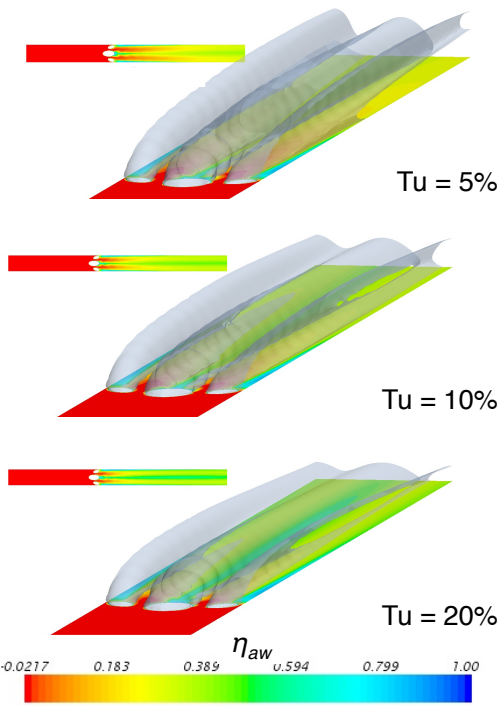

Figure 13. Isosurface of Dimensionless Temperature for Turbulence Intensities of 5, 10 and $20 \%$ 


\section{Conclusions}

In the preliminary study [4] it was found that, at high blowing and density ratios, high freestream turbulence levels increase the film cooling effectiveness of the AVH geometry. Although there are disparities in the data compared to the previous study, the trends and magnitudes remain the same at the same blowing ratio and density ratio. High freestream turbulence does not appear to have any negative effects on the film cooling effectiveness of the AVH geometry at high blowing ratio and density ratio. The AVH geometry has been shown to have a substantial advantage when compared to the baseline cylindrical case from the previous study and work by Heidmann et al [2,3], Hunley et al. [4] and Dhungel et al.[1].

The elevated level of freestream turbulence increases the span-averaged, centerline, and area-averaged film cooling effectiveness for the AVH geometry. The increase in effectiveness coverage extends $30 \mathrm{~d}_{\mathrm{m}}$ downstream of the beginning of the main film cooling hole in the majority of the cases. It is interesting to note that at large length scales and high turbulence intensities there is sufficient mixing far downstream that the effectiveness falls below that of the lower turbulence intensities. Although the heat transfer coefficients are increased with increasing turbulence intensity, it is not to an extent that would decrease the benefit of using the AVH geometry. The NHFR is increased with turbulence intensity in all cases. Two main conclusions can be drawn from the current study:

- It has been shown that the turbulence in the hot gases exiting the combustor can aid in the film cooling for the $\mathrm{AVH}$ geometry at realistic blowing ratios. Heat transfer coefficients were considered but not shown to have enough of an increase to outweigh the benefit of using the AVH.

- Length scale was shown to have little to no effect at the low turbulence level and a small, but noticeable effect at high turbulence intensity. This effect may become more pronounced with non-dimensional length scales $\left(\Lambda_{\mathrm{x}} / \mathrm{d}_{\mathrm{m}}\right)$ closer to engine representative values.

As discussed, future work will investigate more details of the flow physics through further numerical and experimental analysis with the inclusion of more test parameters including approaching boundary layer profiles to the holes and additional blowing ratios and density ratios.

\section{Acknowledgements}

The authors would like to acknowledge the support of the Department of Energy, Office of Science, Experimental Program to Stimulate Competitive Research (EPSCoR) under grant/contract number DE-FG02-09ER46615, monitored by Dr. Tim Fitzsimmons of the DOE Office of Science/EPSCoR.

\section{Nomenclature}

$\mathrm{c}$

blade chord length

$c_{p} \quad$ specific heat at constant pressure

d film cooling hole diameter
DR density ratio $\rho_{\mathrm{c}} / \rho_{\mathrm{i}}$

h heat transfer coefficient

$\mathrm{k} \quad$ turbulent kinetic energy

M blowing ratio or mass flux ratio $(\rho \mathrm{V})_{\mathrm{c}} /(\rho \mathrm{V})_{\text {in }}$

Re Reynolds number

$\mathrm{T} \quad$ temperature

V velocity

$\mathrm{x} \quad$ streamwise direction from the hole leading

edge

y normal direction from the flat plate

$\mathrm{y}^{+} \quad$ dimensionless wall unit

$\mathrm{Z} \quad$ spanwise direction from the hole centerline

Greek adiabatic effectiveness

$\begin{array}{ll}\eta & \text { adiabatic effectiveness } \\ \theta & \text { dimensionless temperature }\end{array}$

$\Lambda_{\mathrm{x}} \quad$ streamwise integral length scale

$\rho$ density

$\omega \quad$ specific dissipation $\varepsilon / \kappa$

Subscripts

aw adiabatic wall conditions

c coolant conditions

in freestream inlet conditions

film local temperatures in freestream affected by

film cooling

$\begin{array}{ll}\mathrm{m} & \text { main hole } \\ \mathrm{s} & \text { side hole or AVH }\end{array}$

wall condition

no film cooling condition

Abbreviations

AVH

CFD

CFD

CMAS

FLOPS

IGCC

anti-vortex hole computational fluid dynamics

counter rotating vortex

calcium-magnesium-alumino-silicate

floating operations per second

IGCC integrated gasification combined cycle

IR infrared

LES large eddy simulations

NHFR net heat flux reduction

PIV particle image velocimetry

RANS Reynolds averaged Navier-Stokes

\section{References}

[1] Dhungel, A., Lu, Y., Phillips, W., Ekkard, S.V., and Heidmann, J.D.,2007, "Film Cooling from a Row of Holes Supplemented with Anti Vortex Holes," ASME Paper GT2007-27419

[2] Heidmann, J.D.,2008, "A Numerical Study of AntiVortex Film Cooling Designs at High Blowing Ratio," ASME Paper GT2008-50845

[3] Heidmann, J.D., and Ekkard, S.V.,2007, "A Novel Anti-Vortex Turbine Film Cooling Hole Concept," ASME Paper GT2007-27528 
[4] Hunley, B.K., Nix, A.C., and Heidmann, J.D.,2010, "A Preliminary Numerical Study on the Effect of High Freestream Turbulence on Anti-Vortex Film Cooling Design at High Blowing Ratio," ASME Paper GT201022077

[5] Repko, T.W., Nix, A.C., and Heidmann, J.D., 2013, "A Parametric Numerical Study of the Effects of Freestream Turbulence Intensity and Length Scale on Anti-Vortex Film Cooling Design at High Blowing Ratio," ASME Paper HT2013-17255

[6] Cumpsty, N., 2003, Jet Propulsion, Cambridge University Press, New York, NY, USA.

[7] Haven, B.A., Yamagata, D.K., Kurosaka, M., Yamawaki, S., and Maya, T., 1997, "Anti-Kidney Pair of Vortices in Shaped Holes and Their Influence on Film Cooling Effectiveness," ASME Paper 97-GT-45

[8] Bunker, R.S., 2005, "A Review of Shaped Hole Turbine Film-Cooling Technology," ASME Journal of Heat Transfer, Vol. 127, pp. 441-453

[9] Rigby, D.L., and Heidmann, J.D., 2008, "Improved Film Cooling Effectiveness by Placing a Vortex Generator Downstream of Each Hole," ASME Paper GT2008-51361

[10] Ely, M.J., and Jubran, B.A.,2008, "A Numerical Study on Increasing Film Cooling Effectiveness through the Use of Sister Holes," ASME Paper GT2008-50366

[11] Leblanc, C., Narzary, D.P., and Ekkad, S., 2013, "FilmCooling Performance of Antivortex Hole on a Flat Plate," Journal of Turbomachinery, Vol. 135, pp. 061009.

[12] Van Fossen, G.J., and Bunker, R.S.,2002, "Augmentation of Stagnation Region Heat Transfer Due to Turbulence from an Advanced Dual-Annular Combustor,"

[13] Wang, H.P., Goldstein, R.J., and Olson, R.L., 1999 , "Effect of High Free-Stream Turbulence with Large Length Scale on Blade Heat/Mass Transfer," Journal of Turbomachinery, Vol. 121, pp. 217-224.

[14] Barringer, M.D., Richard, O.T., Walter, J.P., Stitzel, S.M., and Thole, K.A.,2001, "Flow Field Simulatins of a Gas Turbine Combustor,"

[15] Nix, A.C., Smith, A.C., Diller, T.E., Ng, W.F., and Thole, K.A.,2002, "High Intensity, Large Length-Scale Freestream Turbulence Generation in a Transonic Turbine Cascade,"

[16] Bons, J.P., Macarthur, C.D., and Rivir, R.,1994, "The Effect of High Freestream Turbulence on Film Cooling Effectiveness,"

[17] Saumweber, C., Schultz, A., and Wittig, S.,2002, "FreeStream Turbulence Effects on Film Cooling with Shaped Holes," ASME Paper GT2002-30170

[18] Saumweber, C., and Schultz, A., 2012, "Free-Stream Effects on the Cooling Performance of Cylindrical and Fan-Shaped Cooling Holes," ASME Journal of Turbomachinery, Vol. 127, pp. 061007.
19] Harrison, K.L., and Bogard, D.G.,2008, "Comparison of Rans Turbulence Models for Prediction of Film Cooling Performance," ASME GT2008-51423

[20] Schlicting, H., 1979, Boundary Layer Theory, McGraw-Hill, New York, NY, USA

[21] Goldstein, R.J., 1971, "Film Cooling," Advances in Heat Transfer, Vol. 7, pp. 321-379.

[22] Sen, B., Schmidt, D.L., and Bogard, D.G., 1996, "Film Cooling with Compound Angle Holes: Heat Transfer," ASME Journal of Turbomachinery, Vol. 118, pp. 800806.

[23] Yavuzkurt, S., and Habte, M.,2008, "Effect of Computational Grid Performance of Two-Equation Models of Turbulence for Film Cooling Applications," ASME Paper GT2008-50153

[24] Voigt, S., Noll, B., and Aigner, M.,2010, "Aerodynamic Comparison and Validation of Rans, Urans, and Sas Simulations of Flat Plate Film-Cooling," ASME Paper GT2010-22475

[25] Ekkad, S., 2004, "A Transient Infrared Thermography Method for Simultaneous Film Cooling Effectiveness and Heat Transfer Coefficient Measurements from a Single Test," ASME Journal of Turbomachinery, Vol. 126, pp. 597-603 


\section{APPENDix C: MATLAB CODE FOR EXPERIMENTAL WORK}

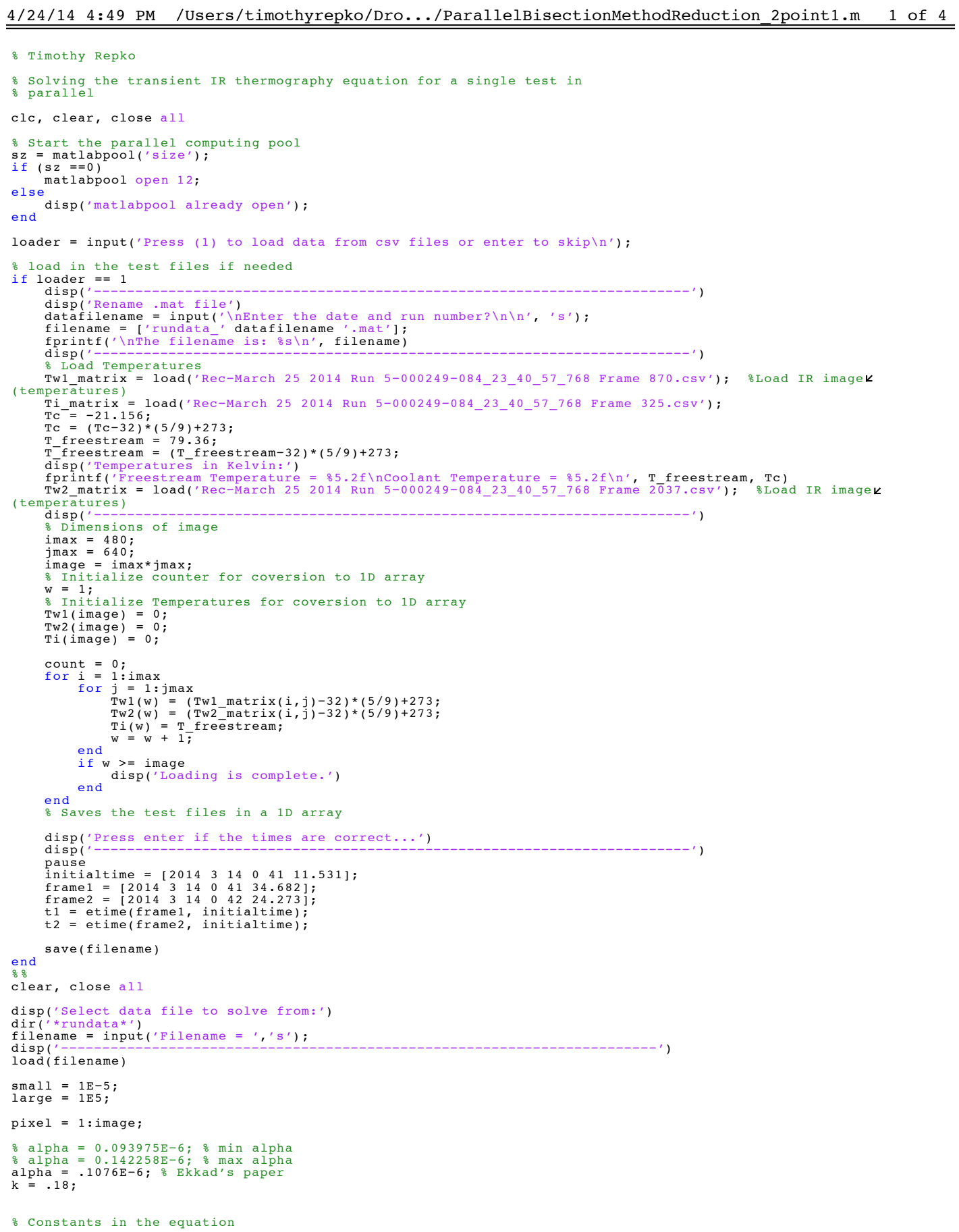


4/24/14 4:49 PM /Users/timothyrepko/Dro.../ParallelBisectionMethodReduction_2point1.m 2 of 4

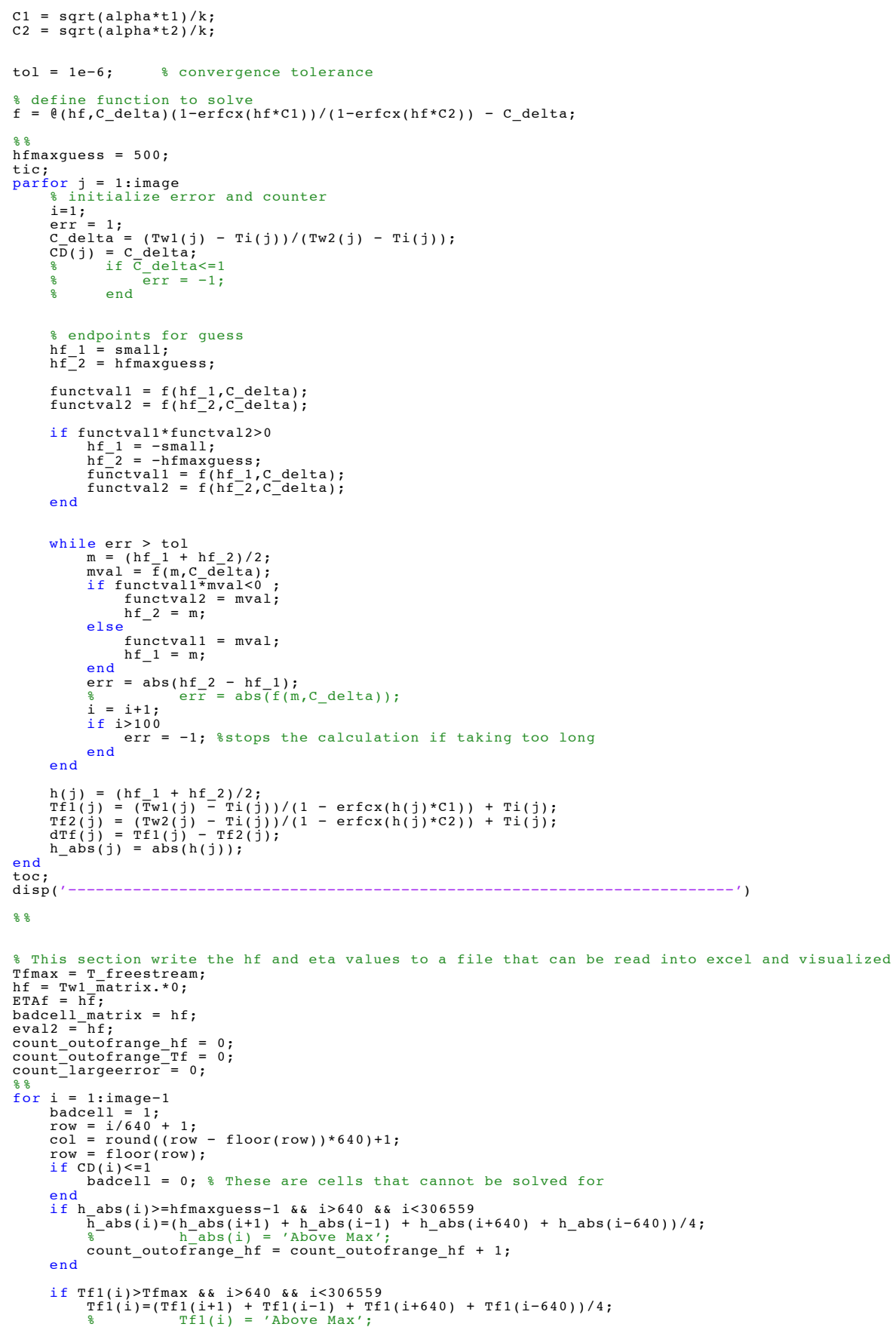


4/24/14 4:49 PM /Users/timothyrepko/Dro.../ParallelBisectionMethodReduction_2point1.m 3 of 4

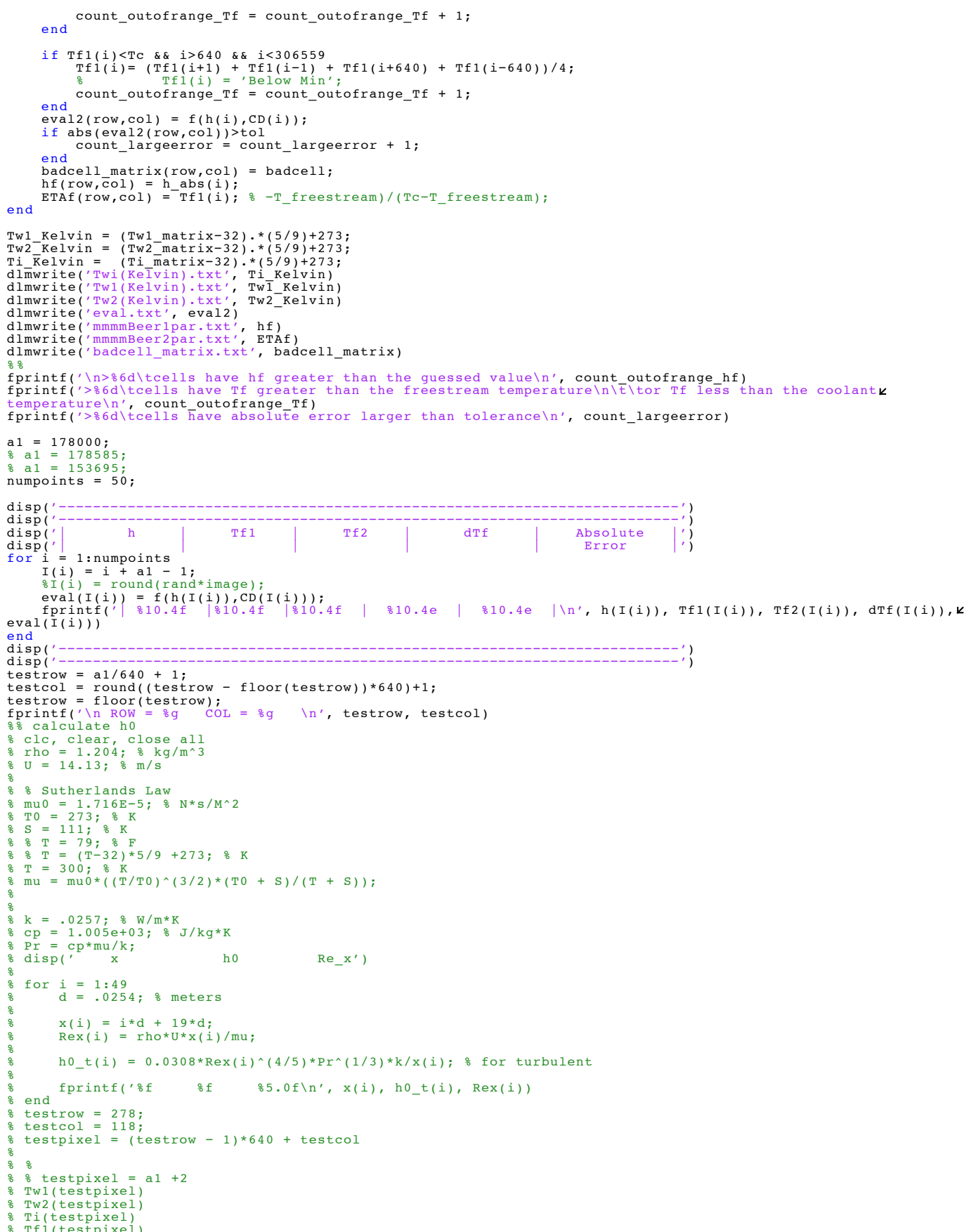


4/24/14 4:49 PM /Users/timothyrepko/Dro.../ParallelBisectionMethodReduction_2point1.m 4 of 4

$\mathrm{h}$ (testpixel)

t 1

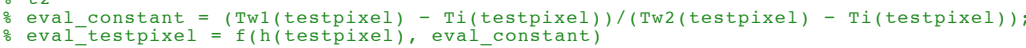




\section{REFERENCES}

[1] Saravanamuttoo, H., Rogers, G., Cohen, H., and Straznicky, P., 2009, Gas Turbine Theory, Pearson Education Limited, Harlow, England.

[2] ASME, Neuchâtel Gas Turbine, February 2014, https://http://www.asme.org/about-asme/who-we-are/engineeringhistory/landmarks/135-neuchatel-gas-turbine.

[3] Solar Turbines, February 2014, https://mysolar.cat.com/cda/layout?m=41108\&x=7

[4] GE Aviation, February 2014, http://www.geaviation.com/engines/military/tf34/

[5] Saunter, M., Clouser, S., and Han, J.C., 1992, "Determination of Surface Heat Transfer and Film Cooling Effectiveness in Unsteady Wake Flow Conditions," AGARD Conference Proceedings 527, pp. 6-1 to 6-2.

[6] http://aeromodelbasic.blogspot.com/2012_01_01_archive.html

[7] Mathieu, J., and Scott, J., 2000, An Introduction to Turbulent Flow, Cambridge University Press, New York, NY.

[8] Libby, P.A., 1974, "On the Prediction of Intermittent Turbulent Flows," Journal of Fluid Mechanics, Vol. 68, pp. 273-295.

[9] Reynolds, O., 1894, "On the Dynamical Theory of Turbulent Incompressible Viscous Fluids and the Determination of the Criterion," Philosopical Transactions of the Royal Society, Vol. A186, pp. 123-164.

[10] Nix, A.C., 2003, "Effects of High Intensity, Large-Scale Freestream Combustor Turbulence on Heat Transfer in Transonic Turbine Blades," Ph.D. Dissertation, Virginia Polytechic Institute and State University.

[11] Dhungel, A., Lu, Y., Phillips, W., Ekkard, S.V., and Heidmann, J.D.,2007, "Film Cooling from a Row of Holes Supplemented with Anti-Vortex Holes," ASME Paper GT2007-27419.

[12] Heidmann, J.D.,2008, "A Numerical Study of Anti-Vortex Film Cooling Designs at High Blowing Ratio," ASME Paper GT2008-50845. 


\section{REFERENCES}

[13] Heidmann, J.D., and Ekkard, S.V.,2007, "A Novel Anti-Vortex Turbine Film Cooling Hole Concept," ASME Paper GT2007-27528.

[14] Repko, T.W., Nix, A.C., and Heidmann, J.D., 2013, "A Parametric Numerical Study of the Effects of Freestream Turbulence Intensity and Length Scale on Anti-Vortex Film Cooling Design at High Blowing Ratio," ASME Paper HT201317255.

[15] Cumpsty, N., 2003, Jet Propulsion, Cambridge University Press, New York, NY, USA.

[16] Han, J.-C., Dutta, S., and Ekkard, S.V., 2000, Gas Turbine Heat Transfer and Cooling Technology, Taylor \& Francis, New York, NY, USA.

[17] Kays, W.M., and Crawford, M.E., 1966, Convective Heat and Mass Transfer, McGraw-Hill, Inc. USA.

[18] Haven, B.A., Yamagata, D.K., Kurosaka, M., Yamawaki, S., and Maya, T., 1997, "Anti-Kidney Pair of Vortices in Shaped Holes and Their Influence on Film Cooling Effectiveness," ASME Paper 97-GT-45.

[19] Ammari, H.D., Hay, N., and Lampard, D., 1990, "The Effect of Density Ratio on the Heat Transfer Coefficient from a Film Cooled Flat Plate," ASME Journal of Turbomachinery, Vol. 112, pp. 444-450.

[20] Bunker, R.S., 2005, "A Review of Shaped Hole Turbine Film-Cooling Technology," ASME Journal of Heat Transfer, Vol. 127, pp. 441-453.

[21] Rigby, D.L., and Heidmann, J.D., 2008, "Improved Film Cooling Effectiveness by Placing a Vortex Generator Downstream of Each Hole," ASME Paper GT2008-51361.

[22] Zuniga, H.A., and Kapat, J.S., 2009, "Effect of Increasing Pitch-Diameter Ratio on the Film Cooling Effectiveness of Shaped and Cylindrical Holes Embedded in Trenches," ASME Paper GT2009-60080.

[23] Ely, M.J., and Jubran, B.A., 2008, "A Numerical Study on Increasing Film Cooling Effectiveness through the Use of Sister Holes," ASME Paper GT200850366.

[24] Hunley, B.K., Nix, A.C., and Heidmann, J.D.,2010, "A Preliminary Numerical Study on the Effect of High Freestream Turbulence on Anti-Vortex Film Cooling Design at High Blowing Ratio," ASME Paper GT2010-22077. 


\section{REFERENCES}

[25] Leblanc, C., Narzary, D.P., and Ekkad, S., 2013, "Film-Cooling Performance of Anti-Vortex Hole on a Flat Plate," ASME Journal of Turbomachinery, Vol. 135, pp. 061009.

[26] Van Fossen, G.J., and Bunker, R.S.,2002, "Augmentation of Stagnation Region Heat Transfer Due to Turbulence from an Advanced Dual-Annular Combustor," ASME Paper GT2002-30184.

[27] Wang, H.P., Goldstein, R.J., and Olson, R.L., 1999, "Effect of High Free-Stream Turbulence with Large Length Scale on Blade Heat/Mass Transfer," ASME Journal of Turbomachinery, Vol. 121, pp. 217-224.

[28] Barringer, M.D., Richard, O.T., Walter, J.P., Stitzel, S.M., and Thole, K.A., 2002, "Flow Field Simulations of a Gas Turbine Combustor," ASME Journal of Turbomachinery, Vol. 124, pp. 508-516.

[29] Bons, J.P., Macarthur, C.D., and Rivir, R., 1996, "The Effect of High Freestream Turbulence on Film Cooling Effectiveness," ASME Journal of Turbomachinery, Vol. 118, pp. 814-825.

[30] Saumweber, C., Schultz, A., and Wittig, S.,2002, "Free-Stream Turbulence Effects on Film Cooling with Shaped Holes," ASME Paper GT2002-30170.

[31] Saumweber, C., and Schultz, A., 2012, "Free-Stream Effects on the Cooling Performance of Cylindrical and Fan-Shaped Cooling Holes," ASME Journal of Turbomachinery, Vol. 127, pp. 061007.

[32] Kim, J., Moin, P., and Moser, R., 1987, "Turbulence Statistics in Fully Developed Channel Flow at Low Reynolds Number," Journal of Fluid Mechanics, Vol. 177, pp. 133-166.

[33] Wilcox, D.C., 1993, Turbulence Modeling for CFD, DCW Industries, Inc., La Canada, California.

[34] Versteeg, H.K., and Malalasekera, W., 2007, An Introduction to Computational Fluid Dynamics: The Finite Volume Method, Pearson Education Limited, Essex, England.

[35] Harrison, K.L., and Bogard, D.G.,2008, "Comparison of Rans Turbulence Models for Prediction of Film Cooling Performance," ASME GT2008-51423.

[36] Mentor, F.R., 1993, "Zonal Two-Equation k- $\omega$ Turbulence Models for Aerodynamic Flows," AIAA Paper 93-2906.

[37] CD-Adapco, 2013, User's Guide. 
[38] Schlicting, H., 1979, Boundary Layer Theory, McGraw-Hill, New York, NY, USA.

[39] White, F.M., 2006, Viscous Fluid Flow, New York, NY, USA.

[40] Incropera, F.P., Dewitt, D.P., Bergman, T.L., and Lavine, A.S., 2007, Introduction to Heat Transfer, John Wiley \& Sons, USA.

[41] FLIR, 2013, ExaminIR User's Guide.

[42] Ekkad, S., 2004, "A Transient Infrared Thermography Method for Simultaneous Film Cooling Effectiveness and Heat Transfer Coefficient Measurements from a Single Test," ASME Journal of Turbomachinery, Vol. 126, pp. 597-603.

[43] Eberly, M.K., and Thole, K.A., 2013, "Time-Resolved Film-Cooling Flows at High and Low Density Ratios," ASME Paper GT2013-95031.

[44] Goldstein, R.J., 1971, "Film Cooling," Advances in Heat Transfer, Vol. 7, pp. 321-379.

[45] Sen, B., Schmidt, D.L., and Bogard, D.G., 1996, "Film Cooling with Compound Angle Holes: Heat Transfer," ASME Journal of Turbomachinery, Vol. 118, pp. 800-806.

[46] Goldstein, R.J., and Taylor, J.R., 1982, "Mass Transfer in the Neighborhood of Jets Entering a Crossflow," ASME Journal of Heat Transfer, Vol. 104, pp. 715721.

[47] Voigt, S., Noll, B., and Aigner, M.,2010, "Aerodynamic Comparison and Validation of RANS, URANS, and SAS Simulations of Flat Plate Film-Cooling," ASME Paper GT2010-22475.

[48] Leylek, J.H., and Zerkle, R.D., 1994, "Discrete-Jet Film Cooling: A Comparison of Computations Results with Experiments," ASME Journal of Turbomachinery, Vol. 116, pp. 358-368.

[49] Sarginson, J.E., Guo, S.M., and Oldfield, M.L.G., 2002, "Flow Visualization of a Converging Slot-Hole Film-Cooling Geometry," ASME Paper GT-2002-30177. 